\title{
Verification of the CENTRM Module for Adaptation of the SCALE Code to NGNP Prismatic and PBR Core Designs
}

Reactor Concepts RD\&D

Dr. Barry Ganapol University of Arizona

In collaboration with: University of Tennessee

Rob Versluis, Federal POC David Nigg, Technical POC

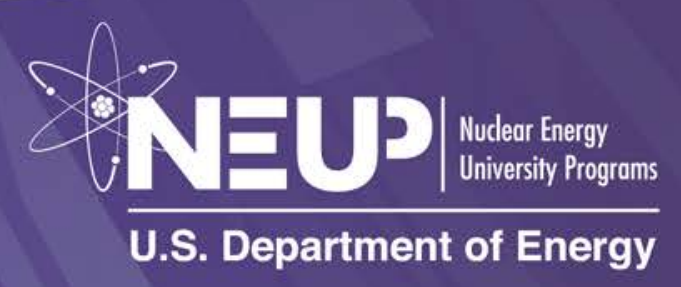




\section{NEUP Final Report: MG/FG/UfG Project}

SUMMARY: The MG/FG/UfG project addresses the question-How can reliable and accurate neutron flux distributions be determined to enable Multigroup(MG), Fine-Group(FG) and Ultra-fine-Group(UfG) cross section processing? The answer, proposed here, is in the development of new and innovative strategies for solving the neutron transport and diffusion equations through the method of panels. These strategies include application of new analytical methods, convergence acceleration, numerical Fourier transform inversion and automated nuclear data access. The intent of this project is to provide a basis for future development that will promote routine use of UfG processing in nuclear reactor physics investigations. While initially focused on verification of the CENTRM code, for reasons to be discussed, the project took on a wider, more basic, view of neutron transport and diffusion theories resulting in new theory, numerical methods, solutions and challenges.

Multigroup/Fine-

Group/Ultra-fineGroup Cross Section Processing

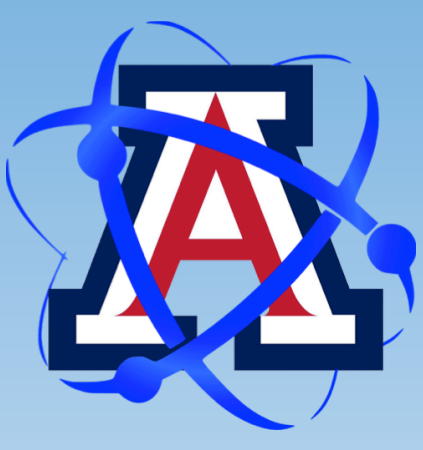


TABLE of CONTENTS

Title Page: NEUP Final Report: MG/FG/UfG Project ..... 3

Abstract (Research Proposed) ................................. 4

Modified Abstract (Research Performed) ...................... 4

I. PROJECT BACKGROUND/SUMMARY/HISTORY .............

1. Project background ...........................................................

2. Project summary ............................................................

2.1. Proposed objective and tasks ...................................

2.2. Modified objective and tasks ......................................

3. Project history ................................................... 10

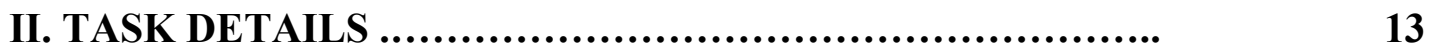

1. TASK 1 ............................................................

1.1. Panel formalism: Infinite medium ..............................

1.2. Generation of ENDF/B cross section data: Format .............

a. ENDF/B Data preparation ............................................

b. UfG processing ................................................................

1.3. Selected results for infinite medium slowing down ..............

1.4. Slowing down in the BL approximation .......................... 35

a. First solution ..............................................................

b. Second solution ............................................................... 36

1.5. Generation of ENDF/B cross section data: Automated ......... 39

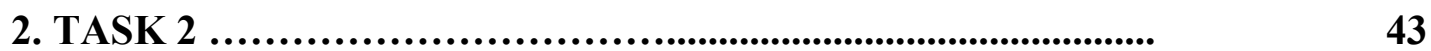

2.1. Theory of the NMG approximation .............................. 43

2.2. Numerical demonstration ...................................... 45

3. TASK 3 ..........................................................................

3.1. Diffusion theory ...............................................................

a. Analytical/Numerical diffusion theory:
MATLAB implementation .........................

a1. Analytical solution ....................................................... 48

a1.1 Application of MATLAB version ........................... 50

a2. Finite difference (FD) solution ................................. 53

b. Analytical/Numerical diffusion theory:
FORTRAN implementation .........................

b1. Analytical solution ...................................................... 57

b1.1. Numerical implementation ................................... 57

b1.2. Numerical demonstration .....................................

b1.3. Remarks ...............................................................

b2. Finite difference solution ........................................

b2.1. Multigroup calculations and comparison to analytical solutions ....................................................

b2.2. Ultra-fine-group calculation for pure slowing down .......

b2.3. Remarks ......................................................................

c. Analytical diffusion theory: GPU implementation ....................... 67 
3.2. Transport theory ...............................................

a. Determination of the multiplication factor $k$...................

a1. Preliminary results ..........................................

a1.1. Single homogeneous medium: Few-group....................

a1.2. Heterogeneous medium ......................................

b. MG/FG/UfG transport solution in an infinite medium ........

b1. Single panel solution............................................

b2. FG/UfG transform solution for an isotropic source ...........

b3. FG/UfG transform inversion for an isotropic source .......... 82

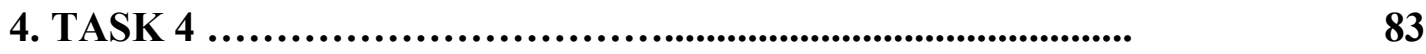

4.1. Preliminaries ............................................................

a. SCALE cross sections ...................................................

4.2. CENTRM/X2 comparison ........................................

4.3. X2/MCNP comparison ...............................................

4.4. Summary .....................................................................

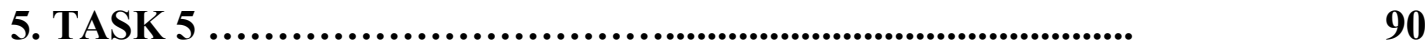

5.1. Analytical fine-group solution in spherical and cylindrical geometries ................................................................... 9

a. Analytical diffusion solutions .....................................

a1. Spherical and cylindrical geometries ........................ 90

a2. Numerical demonstration in cylindrical geometry ............ 92

b. Analytical FD approximation in cylindrical geometry ....... 97

b1. The FD algorithm ..................................................... 97

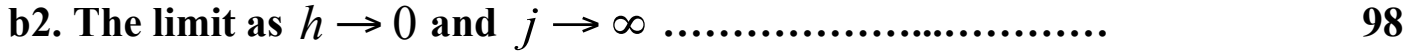

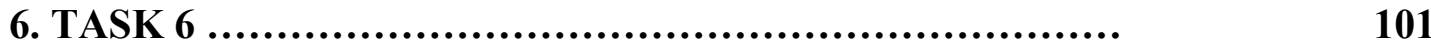

$\begin{array}{ll}\text { 6.1. Burnup mapping with analytical diffusion theory } & 102\end{array}$

REFERENCES ..................................................... 102

III. MODIFIED TASK COMPLETION PERCENTAGES ......

IV. PERSONNEL ...........................................................

V. PUBLICATIONS AND PRESENTATIONS

1 Publications .......................................................... 107

2. Presentations .................................................................. 107

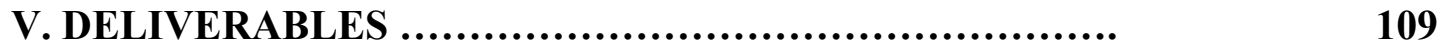

1. UfG/X2 GUI

2. 1D/Analytical diffusion solution

3. 1D/FD diffusion solution

4. Converged accelerated Sn solution 


\section{NEUP Final Progress Report}

Project Title: Verification of the CENTRM module for adaptation to the NGNP prismatic and pebble-bed core designs

(MG/FG/UfG Project)

Duration: $\quad 10.1 .09 / 9.30 .13$

Date of Report: 1.23 .14

Recipient: $\quad$ University of Arizona

Aerospace and Mechanical Engineering Department Rm727 AME Bldg

Tucson AZ 85721

Award Number: DE-F07-09-390

Project Number: 09-390

Principal

Investigator: B.D. Ganapol

Professor

520.621 .4728

Ganapol@,cowboy.ame.arizona.edu 


\begin{abstract}
Research Proposed)
The generation of multigroup cross sections lies at the heart of the NGNP core design whether it is the prismatic (block) or pebble-bed type. The process, generally performed in three steps, is quite involved and its execution crucial to proper reactor physics analyses that follow. Currently, the CENTRM cross section processing module of the SCALE code package has yet to be applied to prismatic or pebble-bed core designs. The primary deliverable of this proposal will be to provide that capability. The deliverable will include a detailed outline of the entire processing procedure for application of CENTRM in a final report complete with demonstration. In addition, the report will include a thorough verification of the CENTRM code. Such a verification has yet to be performed for the CENTRM code, even at ORNL, the originating laboratory. In this way, verification and application are the overall outcome.
\end{abstract}

\title{
Modified Abstract (Research Performed)
}

The generation of multigroup cross sections lies at the heart of nuclear reactor core design. The process, generally performed in three steps, is quite involved and its execution crucial to proper reactor physics analyses that follow. Here, we investigate Multigroup (MG), Fine-Group (FG) and Ultra-fine-group (UfG) algorithms for neutron slowing down through the method of panels. As part of the process, automated cross section data transfer from the BNL/NNDC (National Nuclear Data Center) and subsequent preparation were developed. Efficient data access enabled accurate slowing down algorithms via panels in an infinite medium as well as analytical and finite difference multigroup/fine-group diffusion and transport algorithms. While the method of panels algorithm could not be implemented to the extent initially envisioned, the panel algorithm has been demonstrated for slowing down in an infinite medium and for diffusion and transport in heterogeneous slabs. In addition, the panel algorithm has been theoretically demonstrated in the BL approximation and for spatial transport in an infinite medium as well as for spherical and cylindrical heterogeneous media in analytical diffusion theory. Finally, the algorithm has served as a benchmark for the SCALE/CENTRM code. 


\section{PROJECT BACKGROUND/SUMMARY/HISTORY}

\section{Project background}

The NEUP grant, known as the MultiGroup(MG)/Fine-Group(FG)/Ultra-fineGroup(UfG), i.e., (MG/FG/UfG) ${ }^{1}$, project was motivated by presentations at the DoE/NEUP session held in Salt Lake City in 2008 to introduce future DoE needs to Universities in order to solicit proposals. In particular, D. Petti and H. Gougar indicated, as captured in Fig. 1, the need for new reactor physics methods. The key words in their presentations were "Analytical tools" and "New approaches to slowing down". It was refreshing to see these topics were to be emphasized in NEUP research. The inability of multigroup methods alone to account properly for neutron absorption in resonances has always been an issue in reactor physics analysis. However, in the past, because numerical algorithms were limited by computational speed and storage, we were faced with a zero sum game. If we require more spectral detail, then one must sacrifice spatial or angular detail. Now, with increased computational resources, one can begin to address UfG methods. For increased spectral characterization, success was finally achieved with the CENTRM code [1], developed for the SCALE [2] suite of codes. For the first time, a robust UfG code was developed for practitioners that could routinely output upwards of $70 \mathrm{~K}$ group fluxes in infinite and (now) in 1D media. This was a substantial ORNL project headed by an expert in this area, Dr. Mark Williams. It was apparent, that an independent benchmark could give the CENTRM effort additional credibility. This then became one of the foci of the MG/FG/UfG project proposal submitted by PI B. Ganapol of the University of Arizona. In particular, approximately 20 years ago, the PI had developed a slowing down algorithm that used a method of panels by considering slowing down through segmenting contiguous energy regions-approximately 100 groups each. Consequently, one treats a series of multigroup slowing down problems, rather than one large FG/UfG problem. It was thought that there would be an efficiency in such a procedure. The efficiency would be in computational time and in allowing conventional off-the-shelf codes to be stacked into multiple multigroup calculations to achieve UfG flux distributions. In addition, it was believed that we could incorporate accurate 1D discrete ordinates transport as well as analytical 1D diffusion theory in the panel formalism. However, this could not be accomplished to the extent proposed; nevertheless, with the advances to be described, are very real possibilities considering HPC.

\footnotetext{
${ }^{1}$ If $N$ is the number of groups, then Few-Group is $N<10$; Multigroup $11<N<300$; Fine-Group $301<N<10000$; UfG is $N>10001$.
} 
The initial step in cross section processing is accessing the ENDF/B database for construction of intermediate cross section sets from which one eventually constructs multigroup cross sections by collapsing fine-or ultra-fine cross sections over FG or UfG flux distributions. For this purpose, the CENTRM algorithm was developed as part of the SCALE code package to process granular pointwise

Fig. 1. Power point slides from presentations by D. Petti and H. Gougar indicating a need for a $\mathbf{M G / F G / U f G ~ p r o j e c t . ~}$

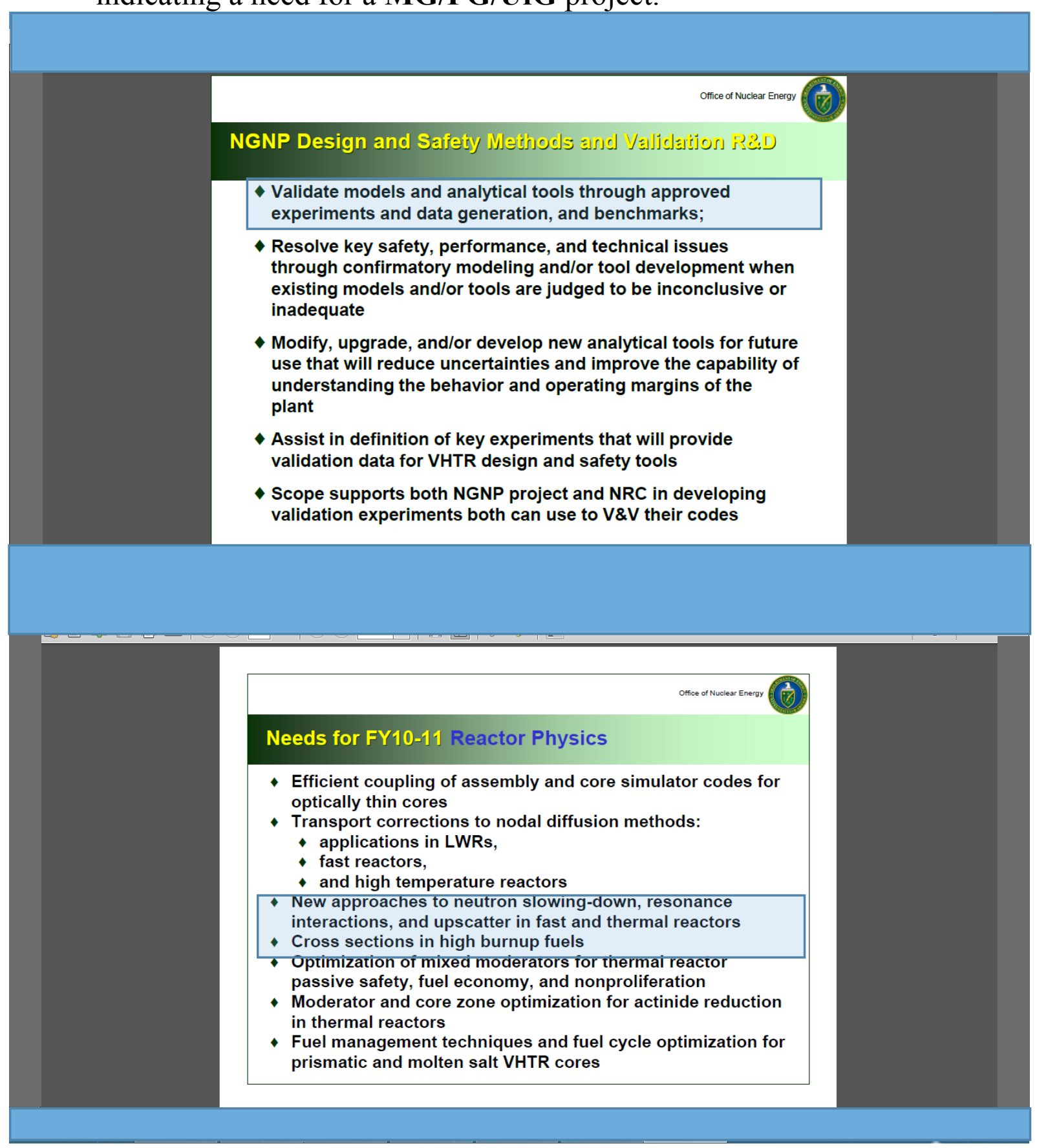


and groupwise cross sections to generate UfG flux spectra. In this way, one includes spectral neutron transport information along with isotopics to establish reactor-specific spectra. In particular, one incorporates the necessary detail to characterize resonance self-shielding by first forming a CENTRM library form AMPX/POLIDENT as a mixture of pointwise and group cross sections in a spectral region. Spatial and temperature information, integrated into the cross section set through transport calculations in an infinite lattice, result in a UfG flux distribution. The ultra-fine flux is then used to collapse to the multigroup cross sections for transport or diffusion theory lattice calculations. Finally, these are collapsed to a few groups for a 3D whole core flux calculation-- the final step in the cross section generation process.

\section{Project summary}

While the CENTRM code is the state-of-the-art for cross section generation, there is always need for further verification and improvement. A part of this project therefore concerns independent verification. An entirely independent method of determining the flux distribution for a large number of energy groups, more than 70,000, is proposed and tested with regard to scaling. In our proposed concept, we form manageable panels of less than 100 groups each to expedite a UfG solution. This concept is tested in the high energy slowing down region were panels are a natural way to treat spectral transport. We begin with the infinite medium (zero dimensions) and progress to 1D- geometries. The key feature is the decomposition of a large number of initial groups into smaller, more manageable, sub-groups. In this way, $N$, the number of groups, where $N$ could be as large as 100,000 required in the resonance region, is decomposed into an additive number of $G$-group problems where $G<<N$. Of course, we can most readily accomplish this in the purely slowing down region, where panels are coupled only through down scatter--but what about the inclusion of upscatter? In this case, we envision an iteration through the panels - a strategy that adjusts to the spectrum as needed because of the panel format. Iteration, however, is left to the future.

We initially demonstrate scaling of the solution for slowing down in multiresonances without spatial variation. Included in the demonstration, is a comparison to CENTRM infinite medium results as an independent verification. Before considering spatial variation in the panel formalism, we derive new analytical solution to the BL equations suitable for panels. We then attempt to adapt analytical diffusion theory into the panel method. While this was only partially successful, we were able to demonstrate the analytical approach for multigroup/fine-group (MG/FG) calculations, which had not previously been 
accomplished. The next phase attempts a 1D transport solution in the infinite cell approximation in plane geometry. We propose a converged accelerated neutron transport algorithm based on discrete ordinates. The formulation, including anisotropic scattering, uses the concept of convergence acceleration to provide at least five-place accuracy. While, this is possibly more accurate than required for routine reactor physics analysis, such accuracy may be important in future reactor physics applications and certainly presents a challenge. Accelerated convergence is based on the diamond difference approximation to the fully discretized transport equation. When each discretization in space and angle is considered an element in a sequence that eventually should converge to the desired solution in the limit of zero discretization and an infinite number of directions, the Wynn-epsilon $(W-e)$ and/or Richardsons algorithms can accelerate convergence. Consequently, a solution is viewed as a sequence of discretized solutions to be extrapolated to their limit. This concept provides high accuracy and is essentially independent of the choice of discretization scheme. Converged accelerated transport, however, could only be applied to the multigroup approximation, but continues as a part of active FG/UfG research. A Fourier transform inversion in an infinite medium however, has been derived and shows promise for FG transport.

In the process of developing the analytical and numerical solutions described above, the MG/FG/UfG team has also developed automated cross section transfer capability, an energy grid selection for multimaterials and initiated a burnup application.

\subsection{Proposed objective and tasks}

Based on the initial proposal and the status of the NGNP project at the start of the grant, the following was the originally proposed objective:

Provide an independent verification of the CENTRM Ultra-fine-Group (UfG) processing code applied to the $\mathbf{N G N P}$ prismatic and pebble-bed core designs.

to be completed according to the following tasks:

1. Thorough testing of the panel algorithm for slowing down.

2. Development of the panel algorithm for multimaterials.

3. Establishment of a multigroup convergence $1 D$ transport acceleration algorithm in the panel formalism.

4. Verification of CENTRM in $1 D$ plane geometry. 
5. Creation and testing of the corresponding transport/panel algorithm in spherical and cylindrical geometries.

6. Formulation of a 2D solution in terms of discretized Green's functions.

7. Application or the verified CENTRM code to current NGNP core design configurations configured in an infinite lattice including Dancoff corrections to simulate Triso/pebble heterogeneity where appropriate.

As with most research projects, the success of the proposed research at the time it is proposed is usually unknown. This was the case for the MG/FG/UfG project. While the success of the panel form for slowing down in an infinite medium was relatively certain, this was not so including spatial variationespecially for transport theory. For this reason, the objective and tasks were modified to include diffusion theory, as this would be nearly as valuable as transport theory and more certain of success. In particular, analytical diffusion theory in 1D geometries had recently been developed by the PI and seemed promising for UfG application-- or more correctly-- for FG application. While this turned out to be true in theory, in practice, the matrix diagonalization, upon which the panel diffusion theory was based, was unstable and the computing time unreasonable. Again, the objective and tasks with respect to the application of panels and spatial diffusion were modified to apply to a single panel only, which is the multigroup or fine-group application. In addition, working with large amounts of data and the CENTRM code proved to be formidable because of the different models used. However, benchmarking was indeed accomplished, but not to the extent originally envisioned. That is to say, in general, the difficulties in achieving exactly what had been initially proposed were underestimated. This coupled with significant bureaucratic delays in hiring and the transfer of funds as well as the change in DoE's NGNP mission led to a request for an additional year at no cost. All of these events contributed to modification of the originally proposed objective and tasks outlined above to (Note: Cross out of the proposed language and addition of new language emboldened)

\subsection{Modified objective and tasks}

Provide an independent verification of the CENTRM Ultra-fine-Group (UfG) processing code applied to the NGNP prismatic and pebble bed eore designs. and develop new analytical/numerical multigroup, finegroup and UfG transport and diffusion methods.

to be completed according to the following modified tasks:

1. Thorough testing of the panel algorithm for slowing down. 
2. Development of the panel algorithm for multimaterials.

3. Establishment of a multigroup/fine-group converged $1 D$ transport (and diffusion) accelerated algorithms in the panel formalism.

4. Verification of CENTRM in $1 D$ plane infinite geometry.

5. Creation and testing of the eorresponding transport diffusion/panel

multigroup algorithm in spherical and cylindrical geometries.

6. Formulation of a $2 D$ solution in terms of discretized Green's functions.

7. Application of the verified CENTRM code to current NGNP core

design configurations configured in an infinite lattice including Dancoff

corrections to simulate Trisolpebble heterogeneity where appropriate.

The last two tasks are to be replaced by

6. Burnup mapping with analytical diffusion theory.

\section{Project history}

In the following, we present a brief history of the events that have shaped the outcome of our NEUP project.

Prior to the start of the grant, a slowing down algorithm had been developed [3] for the verification of the COMBINE spectral code developed over about 30 years at INL. This was an initial attempt using what is called Continuous Analytical Continuation (CAC) to solve the slowing down equation mathematically embedded as a pseudo-time dependent problem. The algorithm appears in Ch. 2 of the PI's book on benchmarks in neutron transport theory [4]. Since the slowing down equations form a lower triangular matrix, the solution is simply a downward cascade, so CAC is considered overkill by some. However, if there is up-scatter this is not the case. Therefore, considering slowing down only, another numerical method had to be constructed, but having CAC as a benchmark provided a consistent verification. The next issue, which turned into major undertaking, was acquiring data since we obviously must go beyond the 167 group COMBINE data in order to proceed to FG/UfG. Fortunately, the BNL/NNDC library was accessible for all data required-but how can the data transfer be accomplished efficiently? It was soon realized that, we would need to transmit hundreds of thousands cross sections quickly and reliably. Initially, the transfer was by hand but in the final year of the grant was automated.

During the first year, it became abundantly clear that the PI could not complete the tasks on his own and additional help was required. Since an appropriate post doc could not be found, the PI reached out to his colleague, Professor Ivan Maldonodo, at the University of Tennessee, Knoxville (UTK), where the PI is a research professor. A seemingly interested student, Shawn Wachter, was 
identified at UTK and a sub-contract was signed and submitted to both universities. For whatever reason, UA did not execute the contract in a timely manner, significantly delaying the effort by about 6 months. In addition, the student identified to complete his Ph.D. under this grant became distracted and was dropped from the project and another interested student, Robby Joseph, was identified. During this period, the PI supervised a visiting student scholar, Elia Battistini, on scholarship from the University of Bologna. Elia began the prototype MATLAB development of the FG/UfG application of neutron diffusion and was instrumental in completing Task 3. In addition, to begin to explore how the cross section transfer could be automated, we hired freshman Blake Tye as a software advisor to see if JAVA could be the preferred language to accomplish data transfer. While, contributing some ideas, unfortunately Blake had some personal issues and had to concentrate on his courses, and subsequently quit the project.

Regardless of the difficulties encountered during the first year, the infinite medium panel formulation and its connection to real data became operational. The next task was to begin verification of CENTRM for an infinite medium since the tool was now in place. The PI intended (quarter time) graduate student Robby Joseph at UTK to complete this work. Unfortunately, due the contract delays, this could not effectively begin until the second year and was one of the reasons a no cost extension (NCE) was eventually requested and granted. After this slow start, work finally began on the verification of the CENTRM code in an infinite medium during the latter part of the second year. Robby, who also worked at ORNL, then became a quarter time active member of the MG/FG/UfG team supervised by his dissertation advisor Dr. I. Maldonado, and reported his first comparison in the third quarter of the second year.

Development and testing of the $\mathbf{U f G}$ algorithm in an infinite medium continued as well as did the development of the FG/UfG formulation of the analytical diffusion solution. As year three came, we began to realize that the analytical solution as formulated would only produce multigroup to fine-group results on the order of several hundred groups without HPC. While this was an advance, it was not UfG. Also, it was realized that a more reliable way of transmitting cross section data was needed. This is when a year's NCE was requested and granted as well as when GPU processing was suggested, which could enable the panel formulation of the analytical diffusion solution. To improve data transfer performance, Dr. I. Guven and a freshman student Michael Futch were added to the team as well a Dr. C. Chan to aid in the theoretical development and eventually to oversee GPU development. In addition, graduate student Aaron 
Farbar, who had GPU experience, became a member of the team. The MAPLE computer algebra package was purchased to resolve the numerical instability of the matrix diagonalization, which is still an ongoing effort. It was during the third year that three undergraduates were added to the project. Two seniors were recruited from the PI's undergraduate reactor physics course as well as an interested freshman. They were given assignments related to the computer algebra formulation of the analytical solution including graphical presentation using MATLAB, MAPLE, and OCTAVE.

In the final year, we see the greatest progress in data transfer with the development of the PYTHON script that essentially spawns the BNL/NNDC site, grabs the required cross section data, brings it local, runs the UfG module X2 and graphs the output. As a result, a manual and four recorded animations have been produced, which are primary deliverables of the project. We also were able to do limited work on the GPU, but never able to achieve the speedup expected. Because of purchasing delays, a larger GPU, whose purchase was approved by DoE, could not be made operational during the project, but is now operational and will continue to be a major part of continuing research. 


\section{TASK DETAILS}

We devote his section to describing progress that has been made on each of the modified tasks. Each will be considered in some detail-most likely, more than a casual reader needs to assess the project's accomplishments. We transmit in this way so that this document represents a full record and serves as a reference for continuing and future MG/FG/UfG research. The mathematical derivations as well as the numerical results as graphs and tables will be inserted inline as appropriate. Much of the detail is taken from conference papers with additional extractions from quarterly reports. It should also be emphasized that the tasks were not performed in chronological order and that more than one task was ongoing.

II.1. TASK 1 Thorough testing of the panel algorithm for slowing down.

\subsection{Panel formalism: Infinite medium}

The panel slowing down description to follow is contained in the A1.SLC.PPT found in the APPENDIX (The APPENDIX is available upon request, all references to the Appendix begin with A\#... .), which is a PowerPoint presentation presented at the NEUP/NGNP Cooperative Methods R\&D Workshop whose objectives are stated on Slide 1. (Note: Some of the key slides are reproduced here so as not to duplicate the reporting effort.)

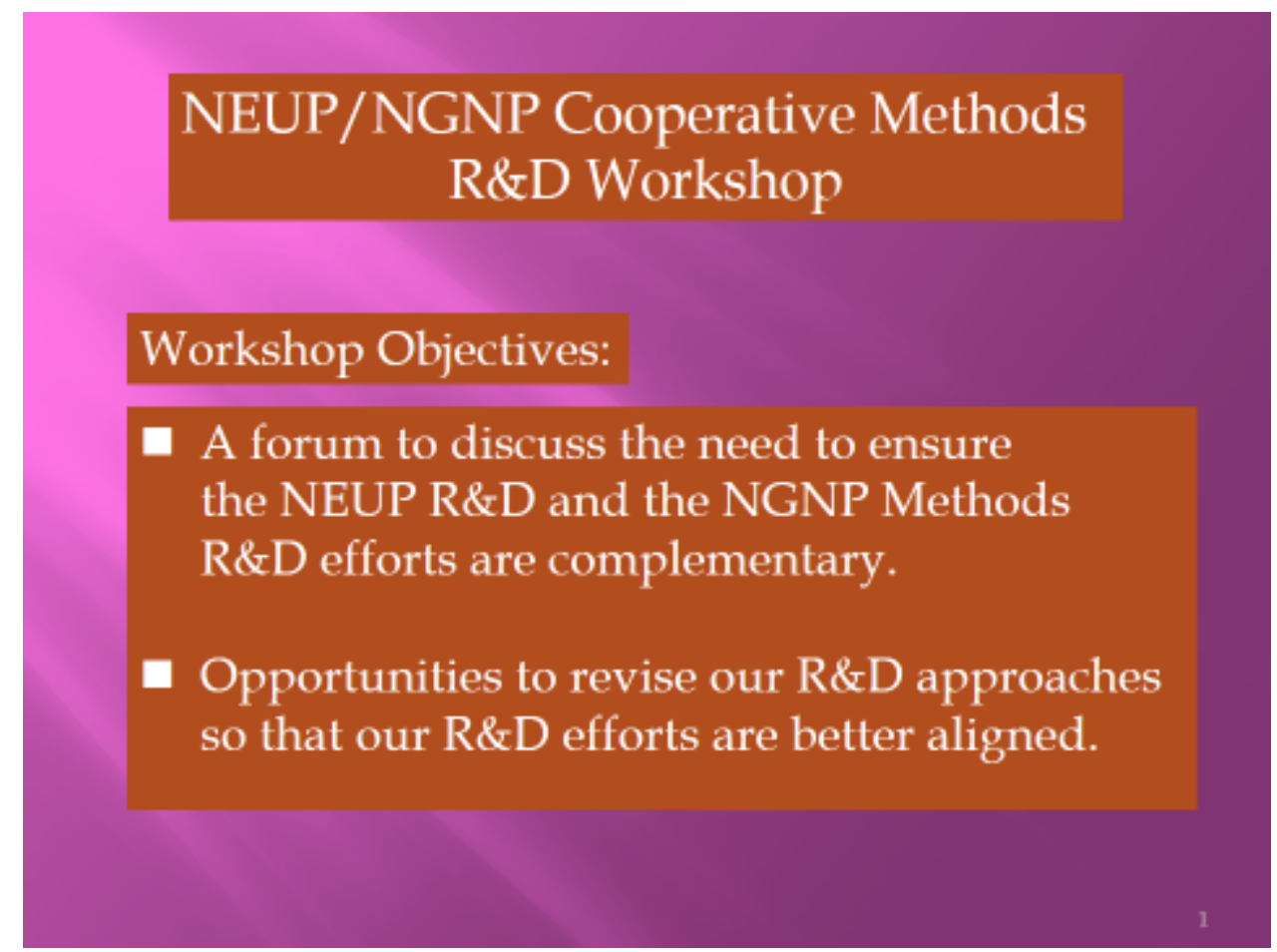

Slide 1 
The general slowing down equation in an infinite medium, which is our focus, is shown on Slide 2. If we assume a source of neutrons enters a spectral region as shown on Slide 3, then the specific slowing down equation to be solved without fission is shown on Slide 4. This is an integral equation requiring discretization,

\section{口 Preliminaries}

* The slowing down equation $(J$ materials) in an infinite medium

$$
\begin{aligned}
\Sigma(E) \phi(E) & =\sum_{j=1}^{J} \int_{0}^{E_{0}} d E^{\Sigma_{j}}\left(E^{\prime}, E\right) \phi\left(E^{\prime}\right)+ \\
& +\chi(E) \int_{0}^{E_{0}} d E^{\prime} v\left(E^{\prime}\right) \Sigma_{f}\left(E^{\prime}\right) \phi\left(E^{\prime}\right)+Q(E)
\end{aligned}
$$

* Consider the Green's function

$$
\begin{aligned}
& \Sigma(E) \psi\left(E ; E^{\prime \prime}\right)=\sum_{j=1}^{J} \int_{0}^{E_{0}} d E^{\prime} \sum_{j(}(E, E) \psi\left(E^{\prime} ; E^{\prime \prime}\right)+\delta\left(E-E^{\prime \prime}\right) \\
& + \text { Gives solution for any source and for fission }
\end{aligned}
$$

Slide 2

* Consider a more physically relevant case

+ Assume $1 / E$ source above highest energy boundary assuming elastic scattering

\section{$E_{0} / \min \left(\alpha_{j}\right)$}

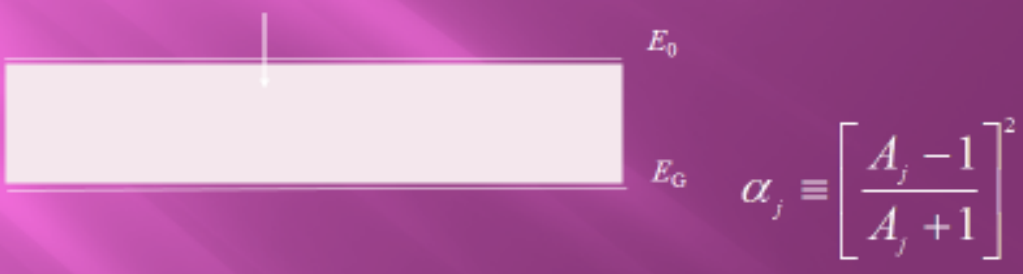

+ Gives consistent $1 / E$ source

$$
Q(E) \rightarrow \sum_{j=1}^{J} q_{0} \int_{E_{0}}^{E_{j} / \alpha_{j}} d E \frac{\sum_{j j}(E, E)}{E}+Q(E)
$$

\section{Slide 3}


* Our focus is the solution of

$$
\Sigma(E) \phi(E)=\sum_{j=1}^{J} \int_{0}^{E_{0}} d E^{\star} \sum_{s j}\left(E^{\prime}, E\right) \phi\left(E^{\prime}\right)+Q(E)
$$

in the UfG approximation

* Possible treatments of cross sections

+ Multipoint

+ Quadrature over energy

+ Multipoint interpolation

+ Multigroup

\section{Slide 4}

which is essentially the multigroup treatment to be chosen. As indicated on Slide 4, there are 4 primary multigroup (MG) treatments. In this project, we exclusively choose the multigroup form, which is described on Slides 5-7. This not to say

MultiGroup approximation (MG) for $J=1$

* Partition energy interval

$$
\Delta E_{g}=\left[E_{g}, E_{g-1}\right], g=1,2, \ldots, N
$$

* Partition integration over energy

$$
\int_{0}^{\infty} d E^{\prime} \Sigma_{s j}(E, E) \phi\left(E^{\prime}\right)=\sum_{g^{\prime}=1}^{N} \int_{\Delta E_{i j}} d E^{\prime} \Sigma_{s j}(E, E) \phi\left(E^{\prime}\right)
$$

* Represent group flux and source by

$$
\begin{aligned}
& \phi(E)=f_{g}(E) \bar{\phi}_{g} \\
& Q(E)=g_{g}(E) \bar{Q}_{g}
\end{aligned} \Longrightarrow \int_{\Delta E_{g}} d E\left\{\begin{array}{l}
f_{g}(E) \\
g_{g}(E)
\end{array}\right\} \equiv 1
$$




\section{Slide 5}

* Group balance equation

$$
\Sigma_{g} \bar{\phi}_{g}=\sum_{g^{\prime}=1}^{N} \Sigma_{s g g^{\prime}} \bar{\phi}_{g^{\prime}}+\bar{Q}_{g}
$$

+ Data: Multigroup parameters

$$
\begin{aligned}
& \Sigma_{g} \equiv \int_{\Delta E_{g}} d E f_{g}(E) \Sigma(E) \\
& \Sigma_{s g^{\prime}} \equiv \int_{\Delta E_{g}} d E \int_{\Delta E_{g^{\prime}}} d E^{\prime} \Sigma_{s}\left(E^{\prime}, E\right) f_{g^{\prime}}\left(E^{\prime}\right)
\end{aligned}
$$

\section{Slide 6}

\section{For $f_{g}(E) \equiv \frac{\alpha_{0 g}}{E} \quad \begin{aligned} & \text { MG parameters are entirely } \\ & \text { analytical }\end{aligned}$}

$$
\begin{aligned}
& \Sigma_{\text {sg' }} \equiv \\
& \equiv \frac{\sum_{g g^{\prime}}}{1-\alpha}\left\{\begin{array}{l}
\Theta\left(E_{g-1}-\alpha E_{g^{\prime}-1}\right) T_{3}\left(E_{g-1} / \alpha, E_{g^{\prime}-1}\right)+\Theta\left(E_{g-1}-\alpha E_{g^{\prime}}\right) T_{3}\left(E_{g^{\prime}}, E_{g-1} / \alpha\right)- \\
-\Theta\left(E_{g}-\alpha E_{g^{-1}-1}\right) T_{4}\left(E_{g} / \alpha, E_{g^{-1}-1}\right)-\Theta\left(E_{g}-\alpha E_{g^{\prime}}\right) T_{4}\left(E_{g^{\prime}}, E_{g} / \alpha\right)- \\
-\Theta\left(E_{g-1}-E_{g^{-1}-1}\right) T_{1}\left(E_{g-1}, E_{g^{\prime}-1}\right)-\Theta\left(E_{g-1}-E_{g^{\prime}}\right) T_{1}\left(E_{g^{\prime}}, E_{g-1}\right)+ \\
+\Theta\left(E_{g}-E_{g^{\prime}-1}\right) T_{2}\left(E_{g^{\prime}}, E_{g^{\prime}-1}\right)+\Theta\left(E_{g}-E_{g^{\prime}}\right) T_{2}\left(E_{g^{\prime}}, E_{g}\right)
\end{array}\right\}
\end{aligned}
$$

where

$$
T\left(v_{1}, v_{2}\right) \equiv \beta\left[\frac{1}{v_{1}}-\frac{1}{v_{2}}\right]-\gamma \ln \left(\frac{v_{2}}{v_{1}}\right)
$$

Slide 7 
the other treatments are inferior, as they may not be. It only makes the statement that there simply was not enough time in the project to consider all four possibilities in sufficient detail. In reality, the multigroup Lagrange interpolation may indeed be the most efficient, since it includes an error estimate--- a conjecture that needs to be shown however. In the MG form, the MG balance equation results as shown in Slide 6 with the MG transfer cross sections given in Slide 7. The unique feature of the formulation presented is that, if we assume a $1 / E$ weighting spectrum, the MG transfer cross sections are determined analytically. This is unlike most MG evaluations that find the multigroup parameters approximately. This can only be done for media of zero temperature however. The panel concept is now applied to enable the solution of the slowing down equation for upwards of $70 \mathrm{~K}$ groups, which is the $\mathbf{U f G}$ challenge.

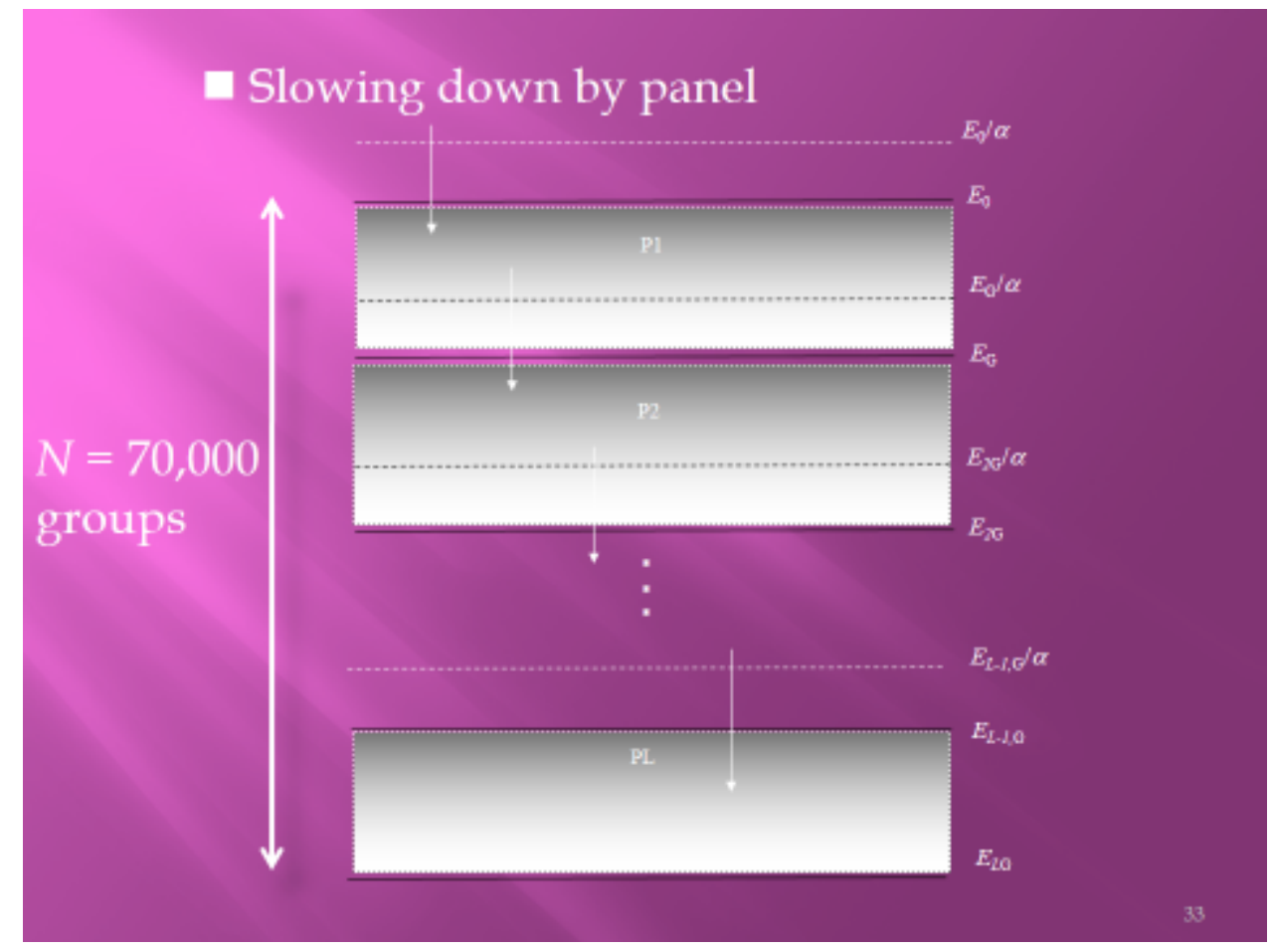

Slide 8

Slide 8 shows the panel configuration, where a source from the adjacent panel above is assumed to feed into the panel below as to form a fixed source for the FG/UfG calculation. The focus now turns to a single panel $l$ as shown in Slide 9. The two neutron sources are external $Q$ and down scatter from region $R$, (defined on Slide 9). The flux is found from the solution to the following set of linear equations: 


$$
\Sigma_{g} \bar{\phi}_{g}^{l}=\sum_{j=1}^{J} \sum_{g^{\prime}=1}^{G} \Sigma_{s j g g^{\prime}} \bar{\phi}_{g^{\prime}}^{l}+\sum_{j=1}^{J} \sum_{r^{\prime}=(l-1) G}^{(l-1) G-R} \Sigma_{s j g r^{\prime}} \bar{\phi}_{r^{\prime}}^{l-1}+\bar{Q}_{g} \cdot(1)
$$

Our initial investigation was primarily concerned with the single material slowing down equation $(J=1)$ for the scalar group flux $\bar{\phi}_{g}^{l}$ in a single panel.

The superscript $l$ refers to an artificial partition of the energy interval of interest into subintervals or panels as shown in Slide 9. The second to last term on the right hand side of $\mathrm{Eq}(1)$ represents the neutron source from panel $l-1$ (or above) slowing down into panel $l$. In this way, we sequentially cover the entire energy range through the linking of panels. In general, one can find numerical solutions to $\mathrm{Eq}(1)$ through the continuous analytical continuation (CAC [1]) formulation or direct solution through matrix inversion. In the CAC form shown in Slide 10, the solution, embedded in a fictitious time-dependent problem, results when we assume a source steady in time. In this case, time serves an iteration index. Taken to steady state then gives the desired slowing down distribution. Further, we resolve the resulting equation through a Taylor series, converged to high accuracy via a Wynn-epsilon ( $W$-e $)$ convergence accelerator [2]. The CAC solution is the most general since it will accommodate upscatter, but only for a single panel. We will consider this approach as an alternative and a verification of what is next proposed. The direct matrix inversion also offers solutions with upscatter, but certainly is not as elegant as CAC.

In first encountering panels in the down scatter approximation, their advantage is not obvious. After all, the pure infinite medium slowing down problem is just a lower triangular matrix requiring only the recurrence of $\mathrm{Eq}(1)--$ what could be simpler. However, complications could arise with recurrence for $N=\sim 100,000$ groups since roundoff error accumulation will eventually destroy the calculation. On the other hand, direct inversion of the full $N \times N$ matrix when $N$ is large is not convenient even in today's large-scale computational environment. Thus, reformulating the solution of the slowing down equation in terms of $N / G$ lower order algebraic equations, where $G$ is the number of groups per panel, presents a reasoned approach. Consequently, the matrix inversion, or convergence to steady state, involves $G$-groups of approximately 100 , rather than $N$-groups on the order of 100,000 .

In principle, by recasting a large slowing down calculation into many smaller ones, potentially the number of groups is unlimited. While we are considering $N$ approximately to be $100 \mathrm{~K}$ here, with faster sequential processors and parallel computing strategies, we could conceive of millions of groups in the future. 
Thus, the proposed algorithm is positioned to be appropriate for even larger slowing down problems.

Since we track the slowing down source, we can include this source via input into existing multigroup codes, which then treats each panel consecutively by stacking now including spatial dependence. Thus, without modification, routine multigroup transport or diffusion codes can generate space dependent FG/UfG weighting spectra directly from down scattering in a panel format.

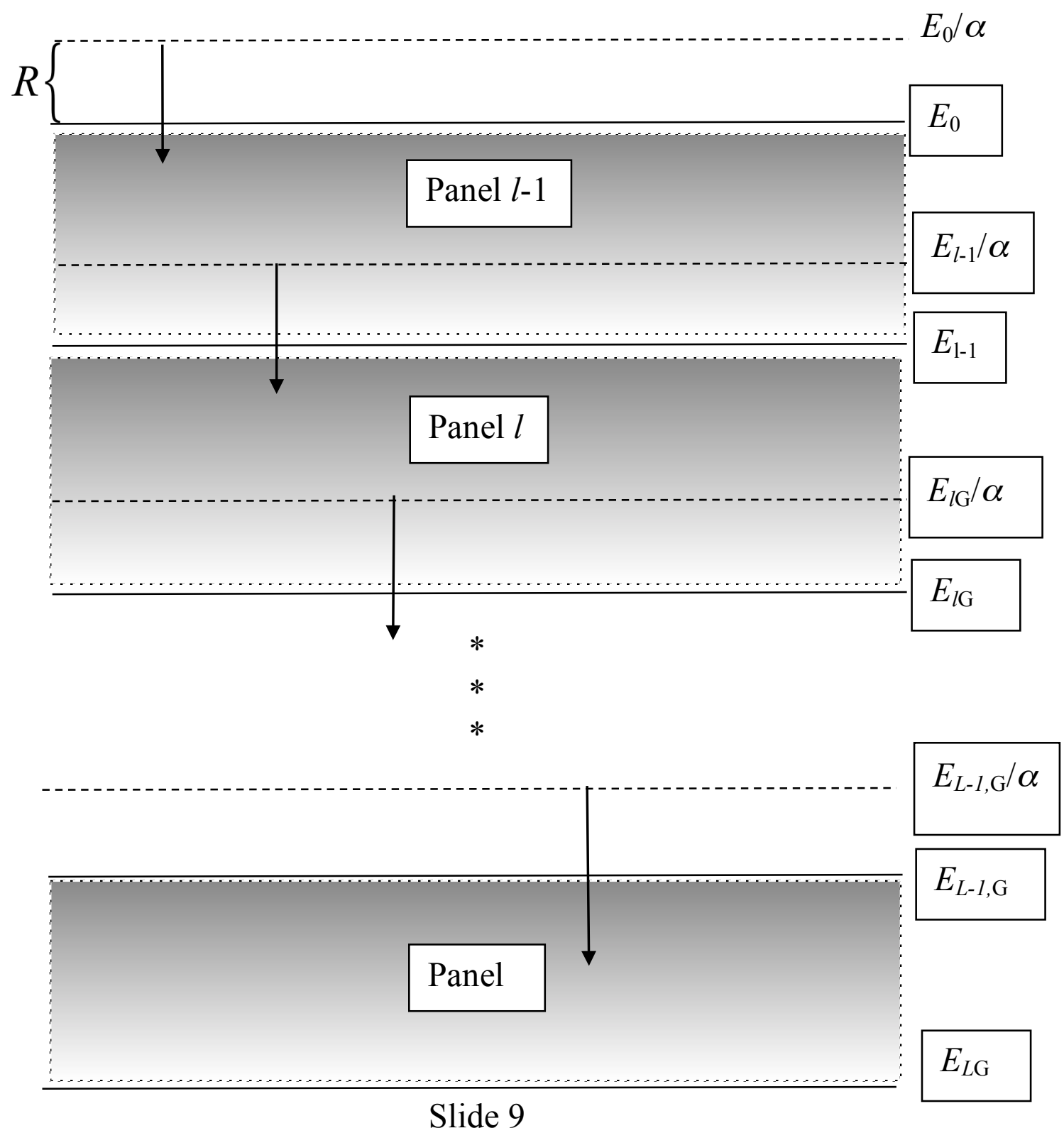


* Solver: THERM/INL

+ Add fictitious time derivative

$\varepsilon \frac{d \bar{\phi}_{g}^{l}(t)}{d t}+\sum_{g} \bar{\phi}_{g}^{l}(t)=$
$=\sum_{g^{\prime}=1}^{G} \sum_{s g g^{\prime}} \bar{\phi}_{g^{\prime}}^{l}(t)+\sum_{r^{\prime}=R}^{G} \sum_{s g r^{\prime}}^{l-1} \bar{\phi}_{r^{\prime}}^{l-1}(t)+\bar{Q}_{g}$

+ Expand in a Taylor series

$$
\bar{\phi}_{g}^{l}(t)=\sum_{k=0}^{K} \bar{\phi}_{k, g}^{l}\left(t-t_{r}\right)^{k}
$$

$+\mathrm{CAC}$

+ Run to steady state

Slide 10

\subsection{Generation of ENDF/B cross section data: Format}

Nuclear data plays a central role in the development of a UFG capability. For this reason, it is imperative that a straightforward connection to the ENDF nuclear data files at BNL/National Nuclear Data Center be a priority. As the research progressed, this phase of development has undergone several implementations, from a "by hand" to automated, as will be shown. The final implementation however, was guided by the format to be described.

\section{a. ENDF/B data preparation}

In this section, we consider accessing ENDF/B cross section data from BNL(NNDC) through the URL

\section{http://www.nndc.bnl.gov/exfor/endf00.jsp.}

When spawned, the URL produces Fig.1. One then follows instructions to choose specific isotopes of interest. As shown in Fig.2, $235 U$ is chosen as the "Target", requesting the neutron total cross section, n,tot for the "reaction" and the cross section, sig, as the "quantity". When submitted, Fig. 3 appears indicating all the possible data resource options. Next, one can plot the ENDF data (Fig.4a) to observe spectral regions of interest. In addition, by selecting (clicking on) a specific region, one can zoom in on a particular energy range. As a demonstration of the ENDF interface, we will concentrate on the start of the resolved resonances, 


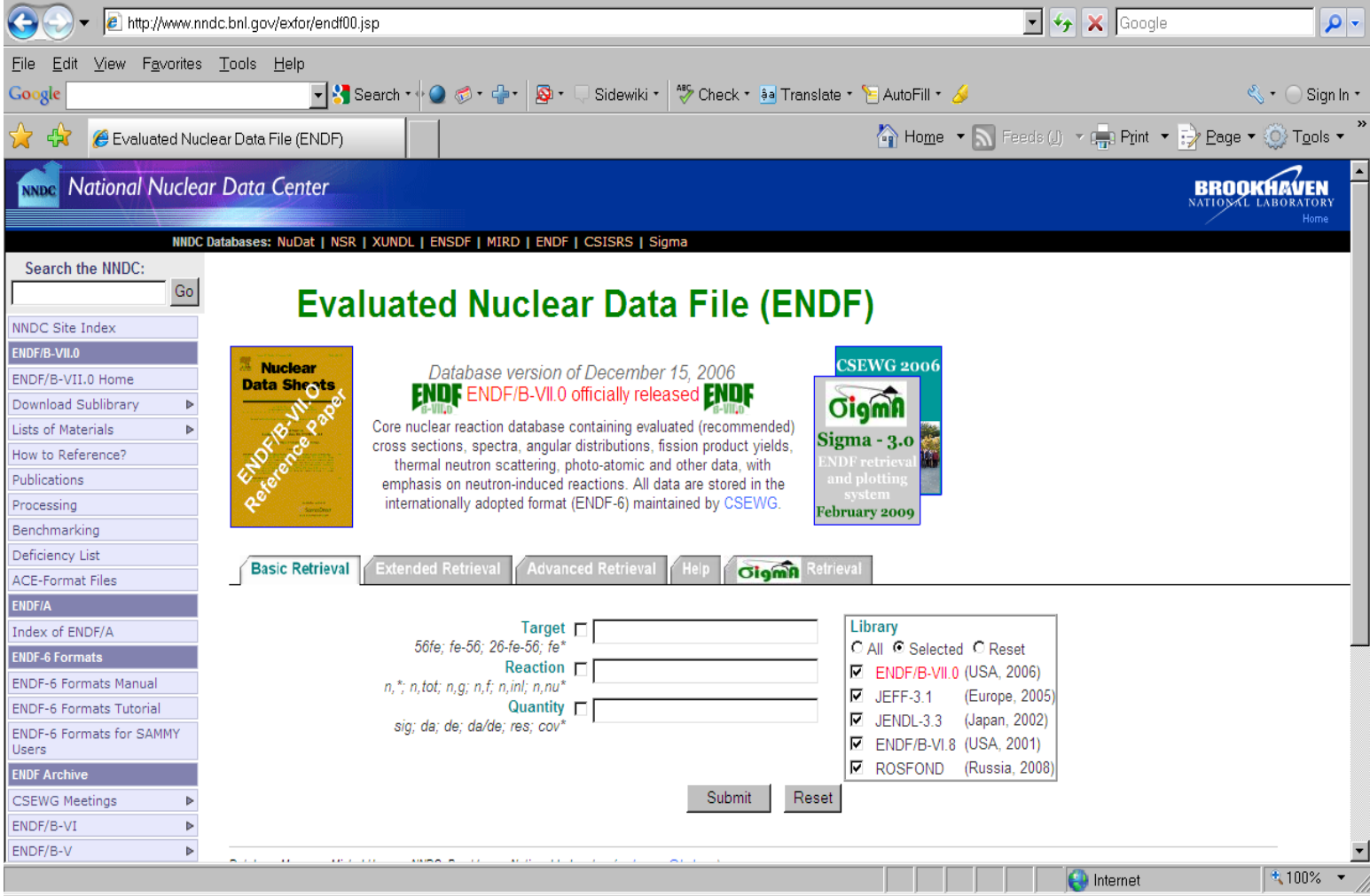

Fig. 1. BNL/NNDC webpage.

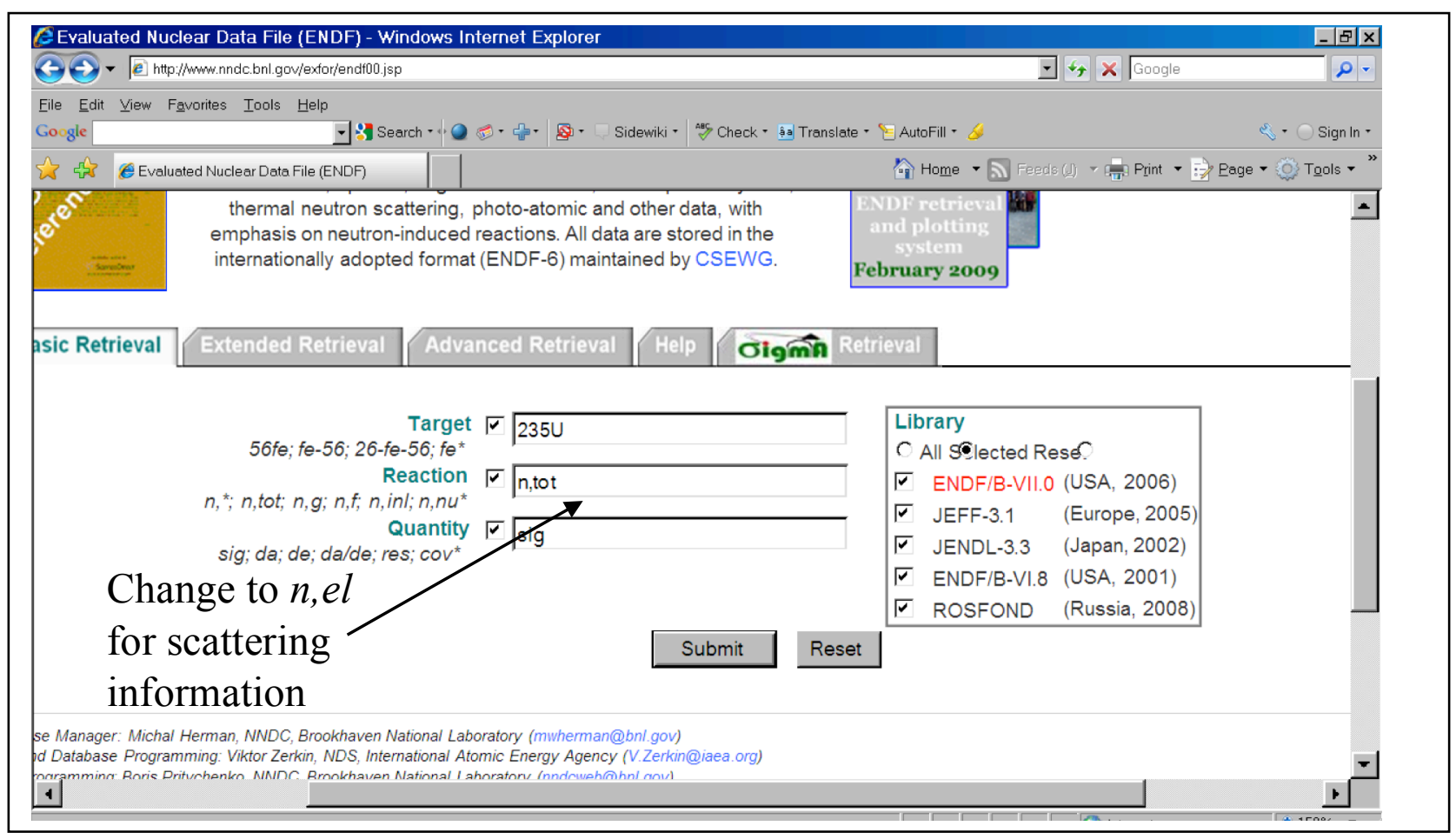

Fig. 2. Choose isotope and reaction. 


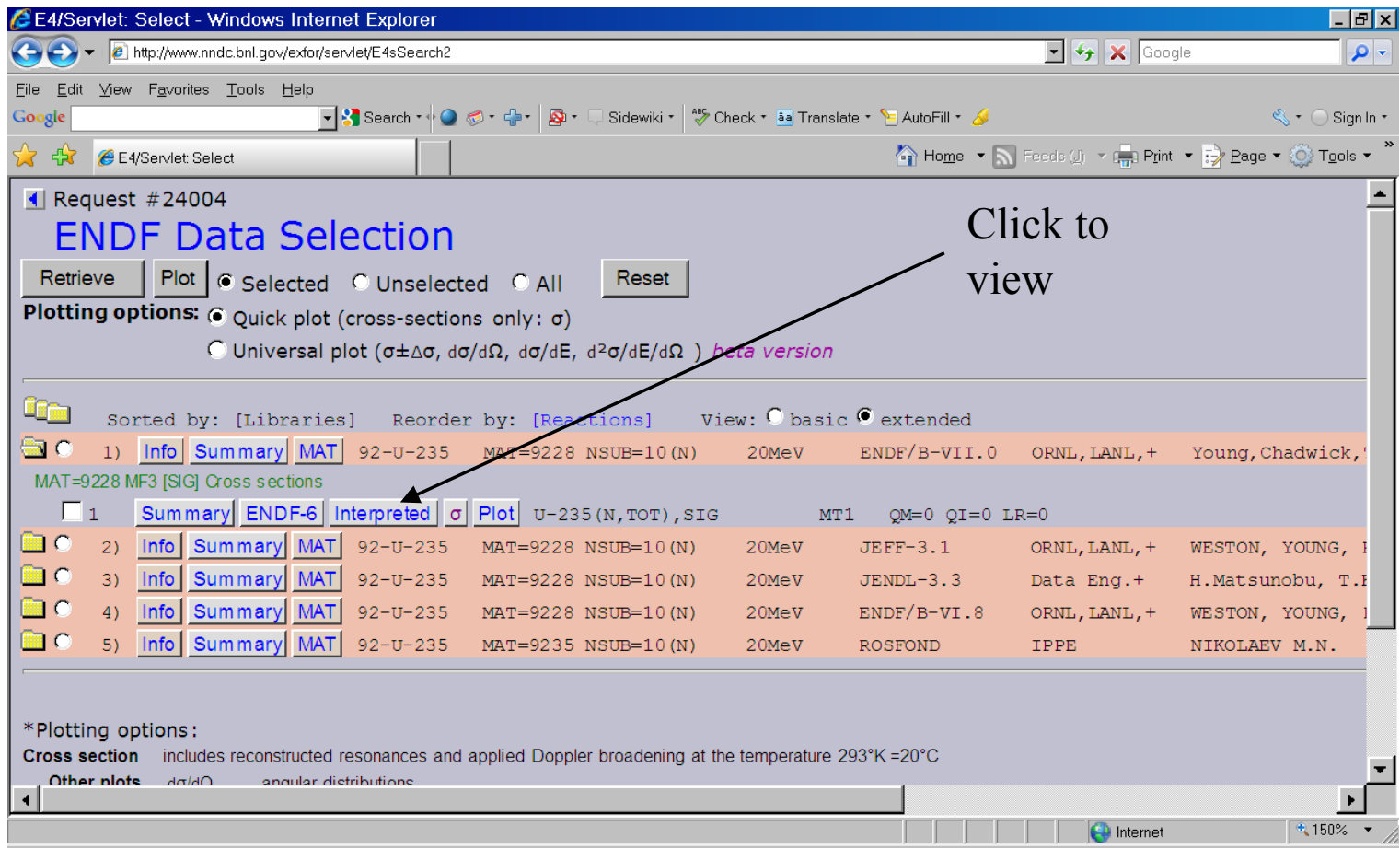

Fig. 3. After submission of choices, indicate which data collection to use.

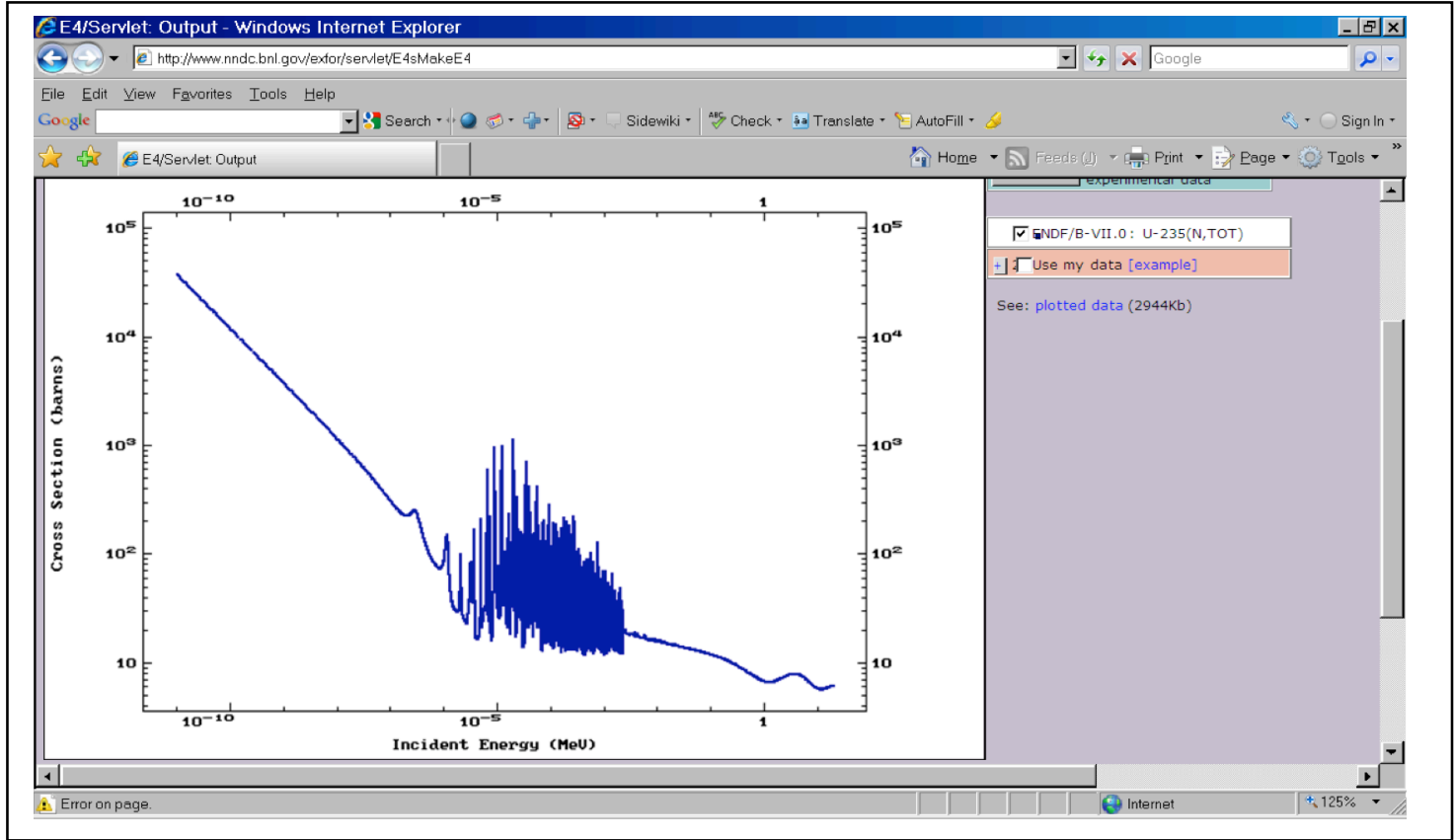

Fig. 4a. Plot data if desired. 


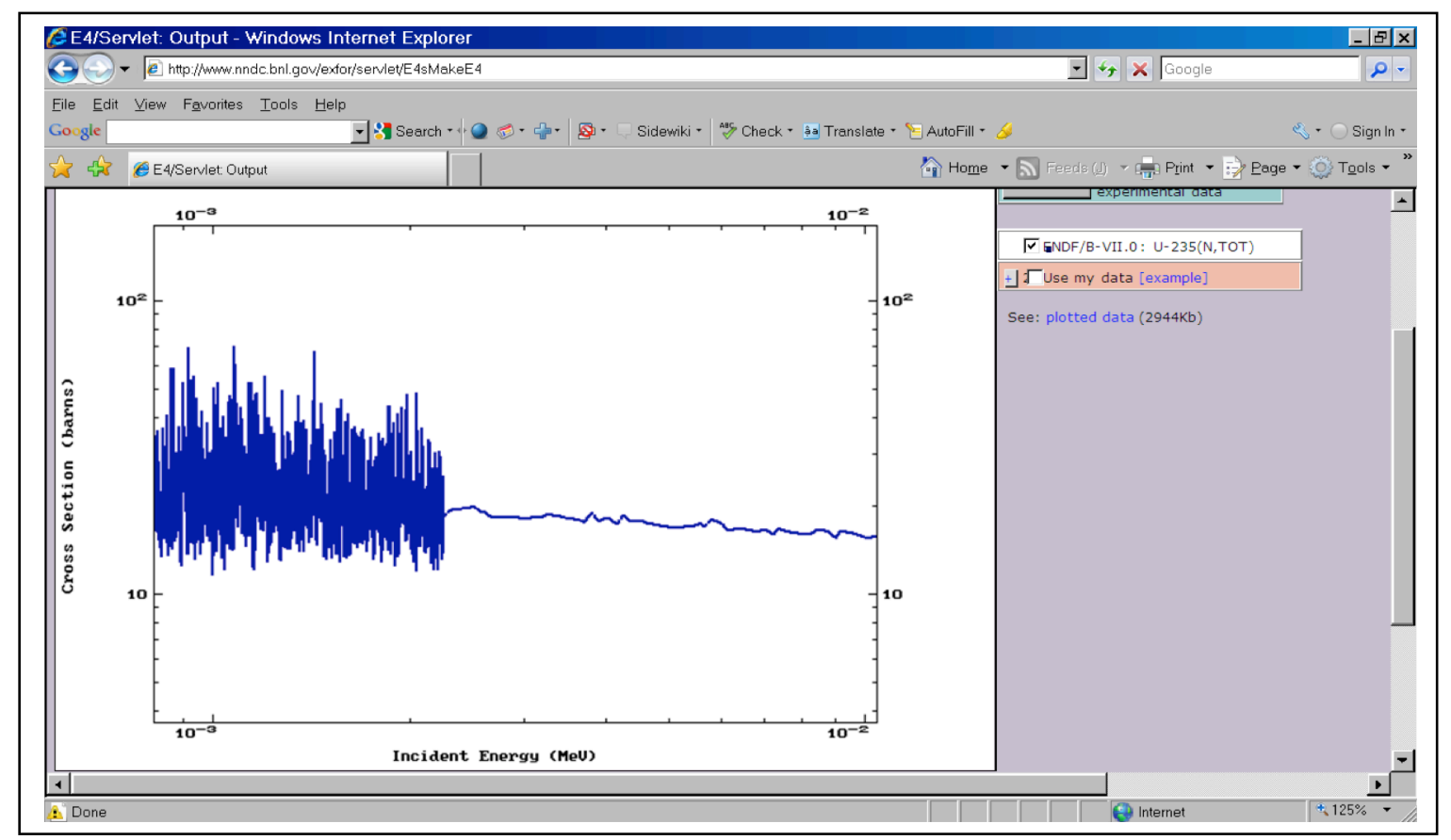

Fig. 4b. Zoom to energies of interest—-start of the resolved resonance region.

$10^{3} \mathrm{eV}$ to $10^{4} \mathrm{eV}$, as shown in Fig. $4 \mathrm{~b}$. To generate a data file of total cross sections for this range only, the $\sigma$ - button (Fig. 3) is clicked to give the following page:

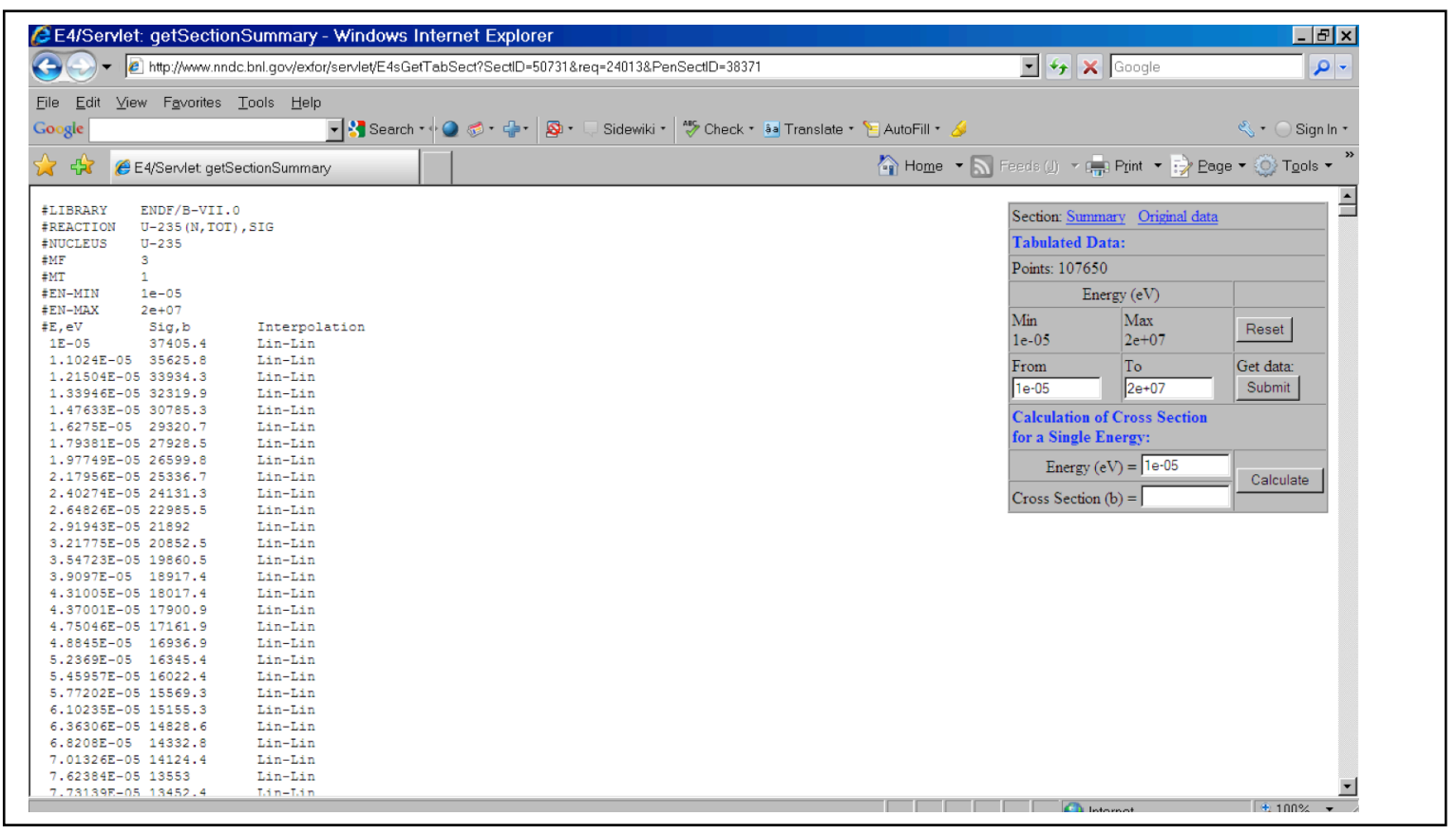

Fig. 5a. Listed cross sections over desired range. 
where the data for the full range is listed on the left and the range choice is on

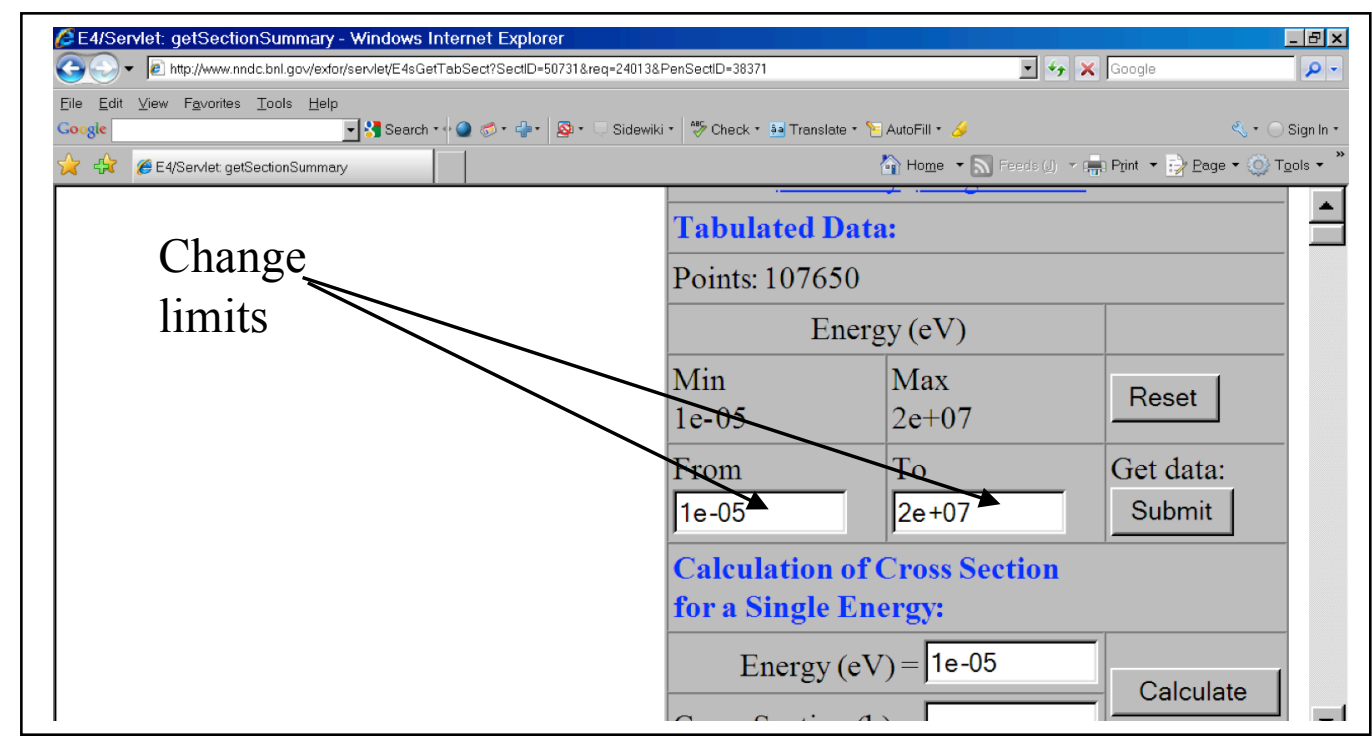

Fig. 5b. Change energy limits to desired range.

the right. We next change the range to our desired range (in $e V$ ) and submit to generate the linearly interpolated cross sections on the left as

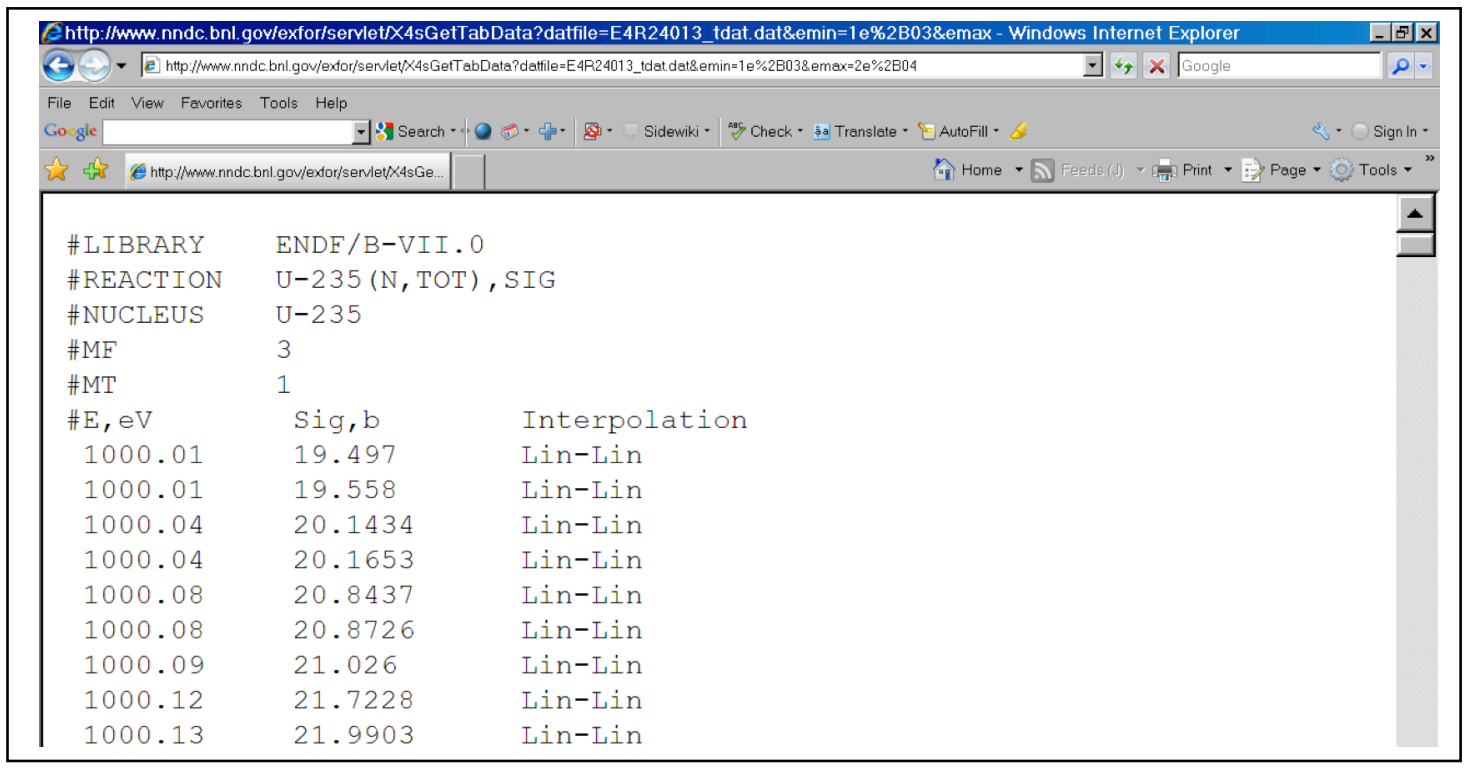

Fig. 5c. Cross sections displayed in range desired.

Finally, the file generated is saved on a secondary file (called Xsec.file) with the following name:

\section{el.atw.int.dat}


where
el
Element/two characters
(eg., UU for U235)
atw
Atomic number/three numbers (eg., 235)
int
Reaction type
(eg., tot or sct for total
or scattering)

The secondary file is saved in the directory to be accessed by the interface (preprocessing) program ENDFPR.f to be described.

The above procedure is repeated for the elastic scattering cross section by replacing $n$, tot in the initial request (Fig. 2) by n,el. All materials required for a particular investigation are found in the above manner and each will then have two (or three with fission) cross section data files (total and scattering) to be input into the next $\mathbf{U} \mathbf{f G}$ processing step.

\section{b. UfG processing}

With the ENDF/B data now collected in an appropriate form for the required materials, we are ready to begin UfG processing. To this end, a pre- processing code has been written to take the ENDF/B data generated and interpolate into the desired groups. The input to the code ENDFPR.f is on file ENDFPR.dat and is given in Fig.6. All the necessary information is provided in the following input description:

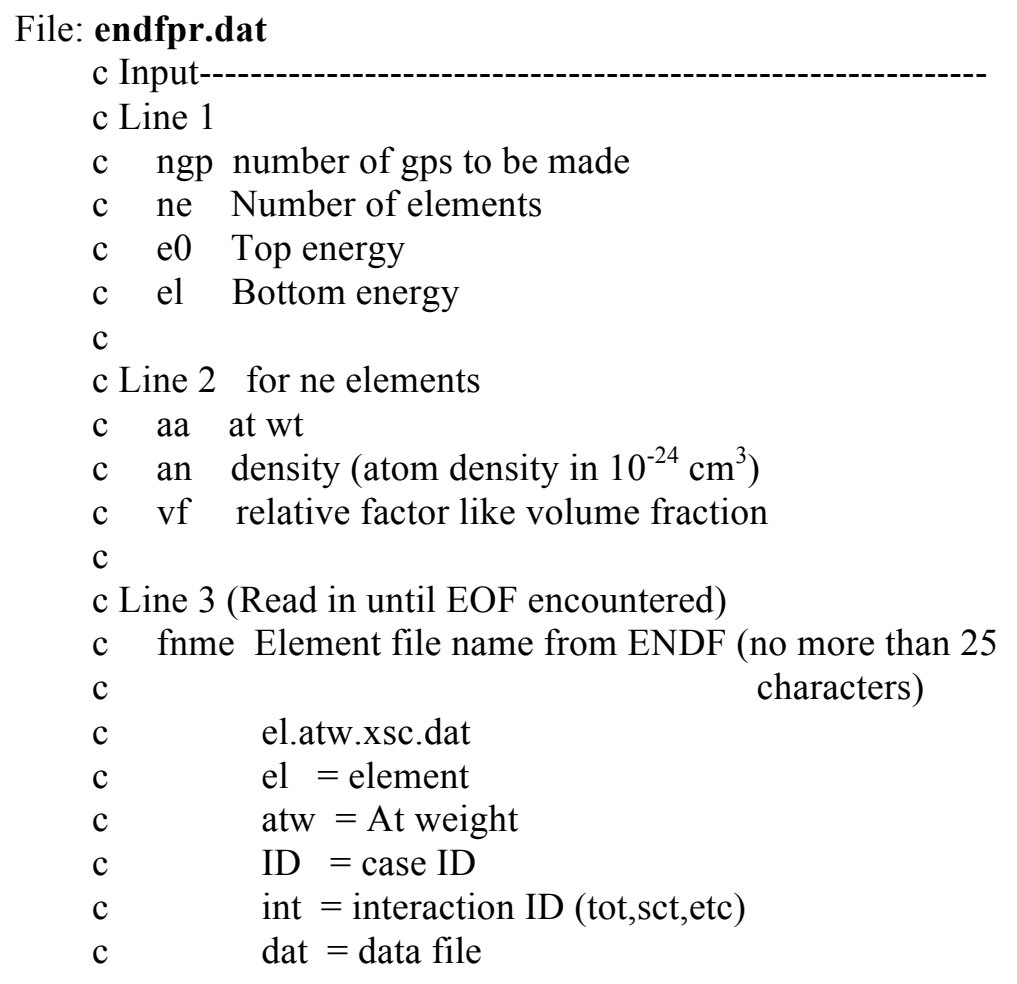


Note that Fig. 6. Input file directive for endfpr.f.

+ No modification of the just-generated data files need be done.

+ Any number of data files can be read in, but each set of two (total and scattering Xsecs) must correspond to an element on line 2.

+ A sub-energy domain of the data stored $(\mathrm{e} 0, \mathrm{el})$ can be requested.

+ The energy range is the minimum top and maximum bottom energies of all requested energies on the Xsec files.

The ENDFPR.f code serves as a linear interpolator of ENDF data to generate a multi- or ultra-fine-group structure. Its output on file o62.dat, in turn, is input to the slowing down program series X2.f that solves the slowing down equation as described in $\S$ II.1.1.

As a demonstration, we consider $\mathrm{U} 235$ in an (infinite) graphite matrix for a series of 10,100,1000 and 10000 groups in the range $1000 \mathrm{eV}$ to $2500 \mathrm{eV}$. The input is

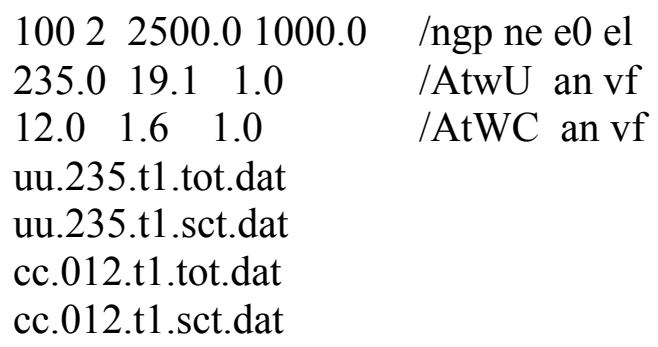

and the output is placed on file o62.dat, which for the case of 100 groups takes the following form:

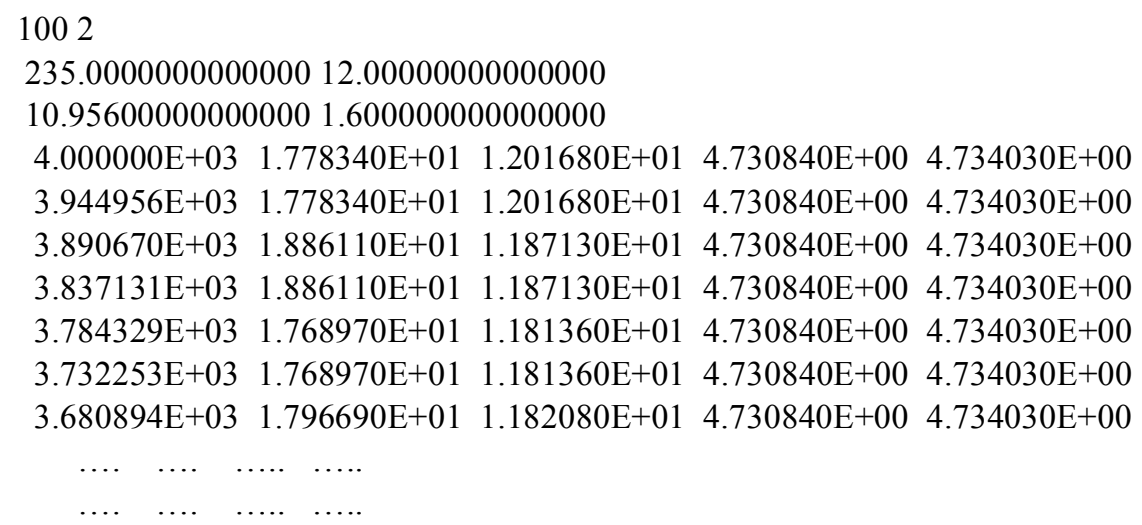

Fig. 7. ENDFPR.f output file o62.dat. 


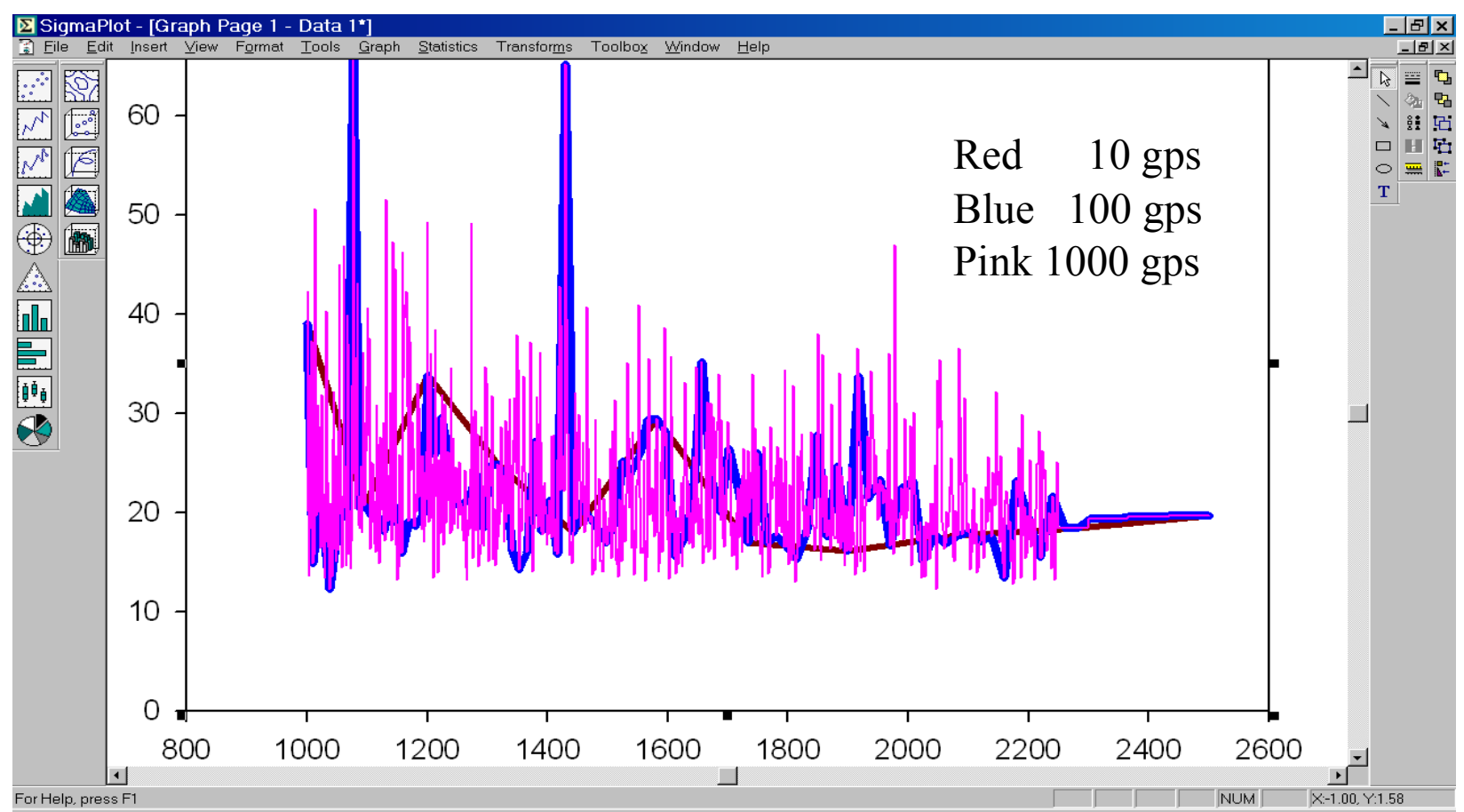

Fig.8a. Xsecs for groups 10, 100, 1000.

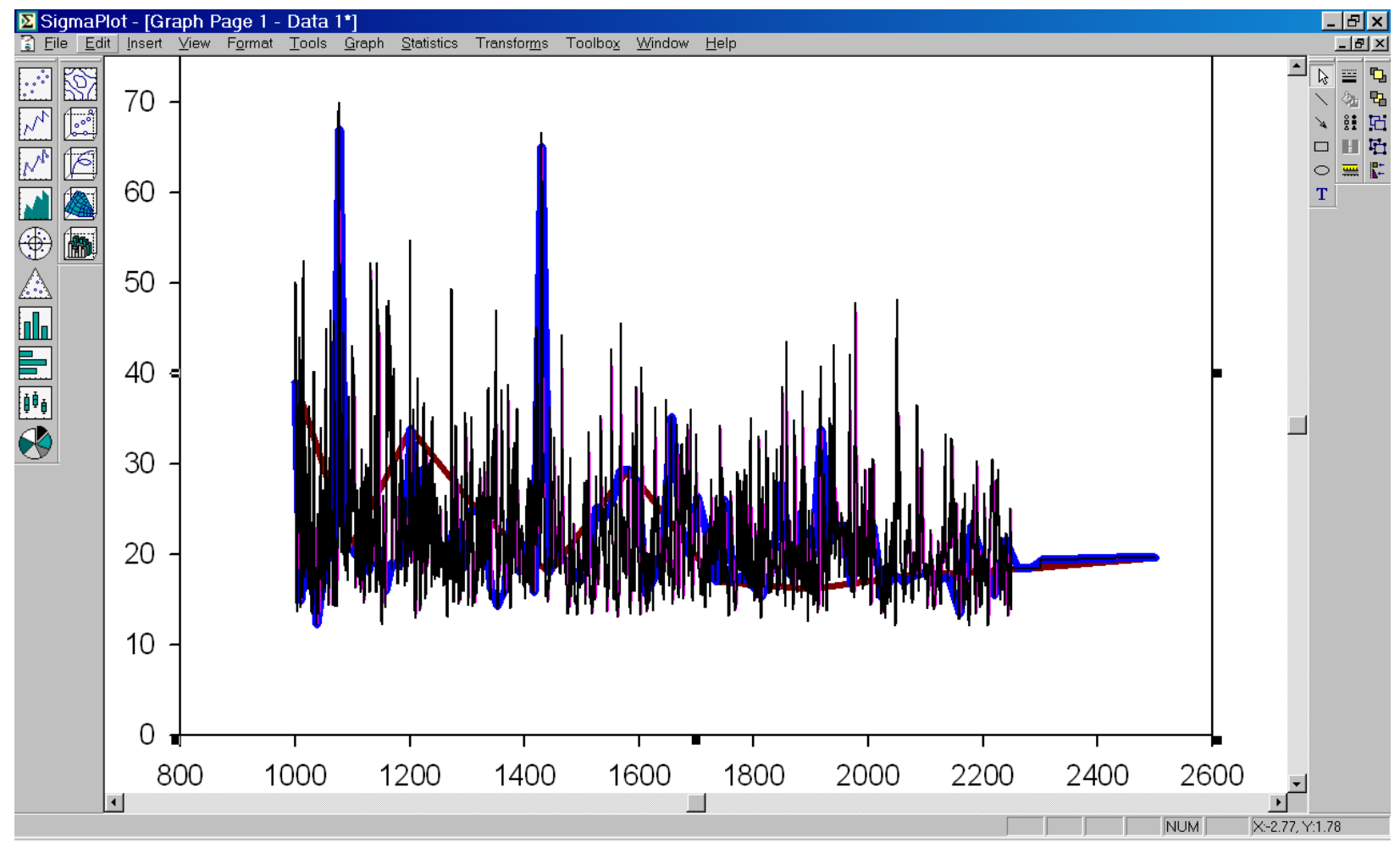

Fig. 8b. Xsecs for above groups in Fig, 8a with the 10,000 group (in Black) case added. 
The first three lines give the desired number of groups and element information. Next, the first column is the energy for the 100 groups taken in equal logarithmic increments over the desired energy range. This is then followed by the linearly interpolated total and scatter cross sections for the two elements. Figures $8 \mathrm{a}, \mathrm{b}$ give a comparison of the granularity of these group configurations. As observed, at least to properly represent this region visually, a large number of groups are required. Of course, the flux approximation is the ultimate indicator of merit.

\subsection{Selected results for infinite medium slowing down}

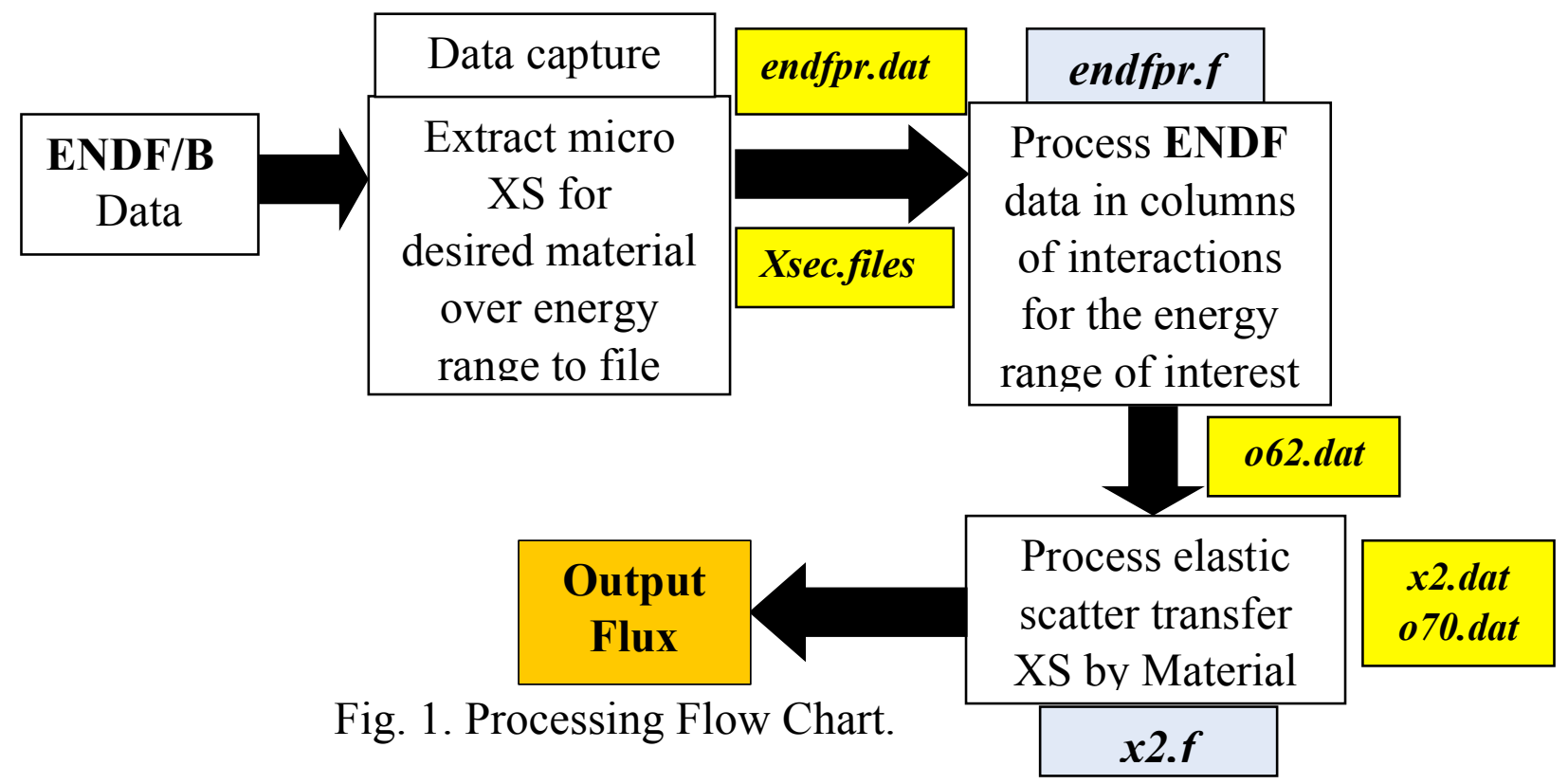

We now numerically solve Eq(II.1.1.1). To this end, Eq(II.1.1.1) is recast as a matrix equation in each panel of the form

$$
A \bar{\phi}^{l}=\bar{q}^{l-1}
$$

for panel $l$. We use LU decomposition [3] to solve this equation for our initial trial. If need be, other more efficient solvers will be tried. A FORTRAN program X2.f has been written for this purpose and is placed second to the end of the data processing stream shown in Fig. 1. Group configurations for $N=10$, $100,1000,5000$ are considered. Following the procedure outlined in $\S I I .1 .1$, the groups were established for slowing down in a homogeneous mixture of U238 and $\mathrm{Na} 23$ in the energy range $0.01 \mathrm{eV}<E<200 \mathrm{eV}$, where several prominent resonances of uranium occur. The variation of the total elastic scattering cross section is shown in the upper plate of Fig. 2. It should be noted that for $N=10$ and 100, the detail of the resonances is hardly apparent and for $N=1000$ and 
5000 , the fine-group configurations are virtually identical. The bottom plate shows the detail of a

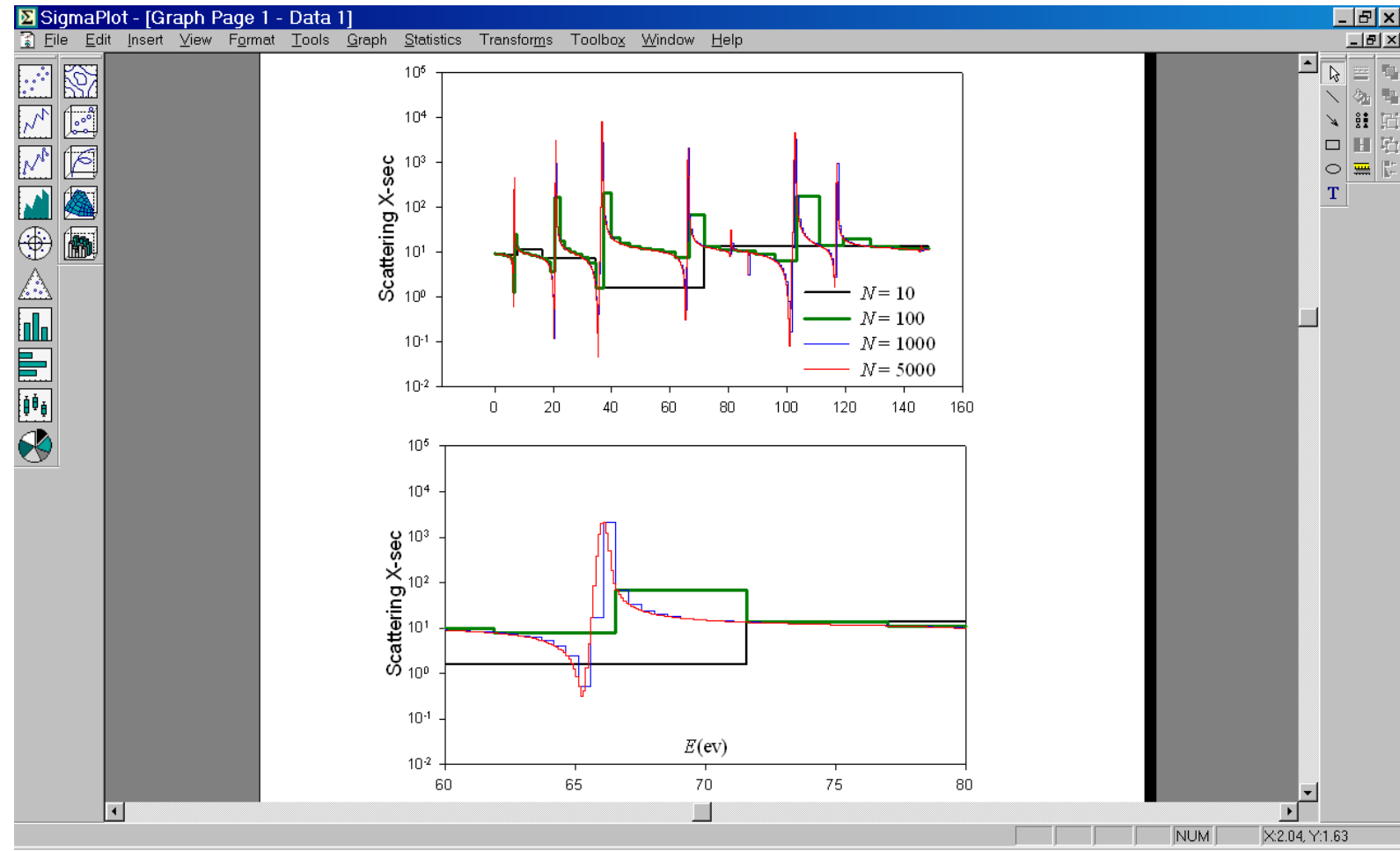

Fig. 2. Scattering Xsec exhibiting the multigroup configuration for test of LU direct solver.

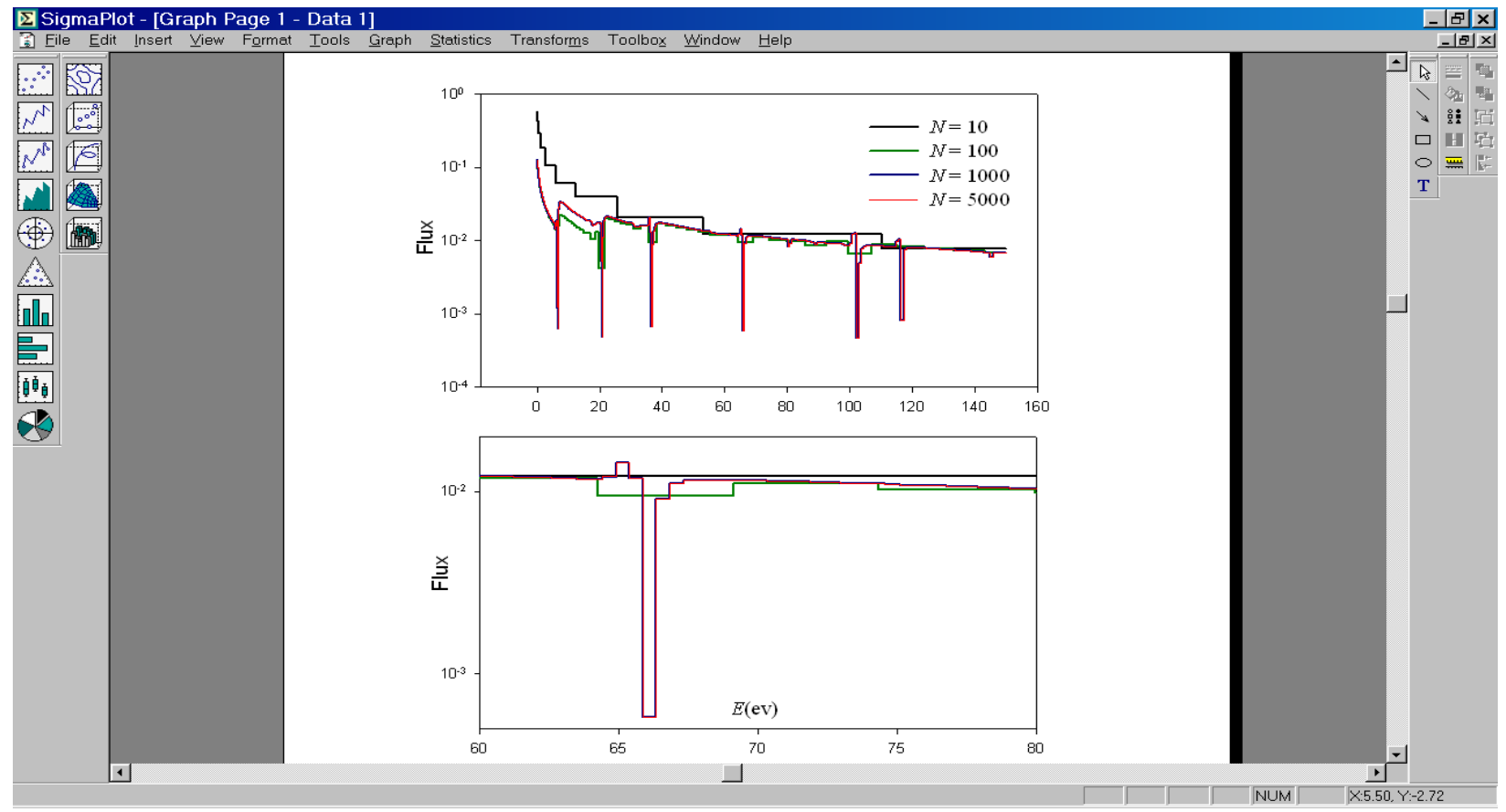

Fig. 3. Flux solutions for multigroup configurations for $G=10$. 
specific resonance, which is covered with adequate fidelity by 5000 (fine) groups. This is a clear indication of the need for ultra-fine-group methods. Figure 3 shows the energy flux variation by group, $\bar{\phi}_{g} / \Delta E_{g}$, for volume fractions 0.9 and 0.1 of $238 \mathrm{U}$ and $23 \mathrm{Na}$ respectively. For each calculation, $G$, the panel group interval, was fixed at 10. Even for $N=10$, the general trend of the flux variation is followed. For the last two configurations, the flux is nearly identical indicating a visually converged solution with respect to the number of energy groups. The bottom plate shows an enlargement on a single resonance.

The cumulative absorption on down scattering of all the resonances requires a sufficient number of groups for accuracy as indicated by the difference between the $N=100$ and 1000 cases.

From this basic investigation, we conclude that the panel method is viable and, more importantly, an enabling method simplifying a calculation that would normally be relatively difficult by conventional means. The computational time for these examples was about $10 \mathrm{~s}$ on a $1.2 \mathrm{GHz}$ Gateway laptop, which is not yet fast enough.

Finally, to demonstrate a true UfG calculation, 30K groups were considered over the specified energy range. Figure 4 shows the flux variation with composition of the fuel and absorber. All cases were for 3000 panels $(G=10)$. The increase in flux depletion with increasing amount of fuel is quite apparent. The bottom plate is a zoom on several resonances showing all their essential features.

As another demonstration, the nominal case of $0.9 / 0.1$ was performed with $G=$ 10(10)100. Identical results to all digits and all panel intervals $(G)$ were obtained with an increase of $24 s$ to $29 s$ over the $G$-variation. Again, the computational time must be reduced for this algorithm to be competitive. Computational time however, is not as important as the benchmarking value this algorithm brings at this point.

We present additional results in A1.SLC.PPT to be summarized as follows:

Slide 1 gives a simple verification of the slowing down treatment, where a localized energy source in Carbon produces the anticipated $1 / E$ flux after an initial inverted Plazcek transient. 


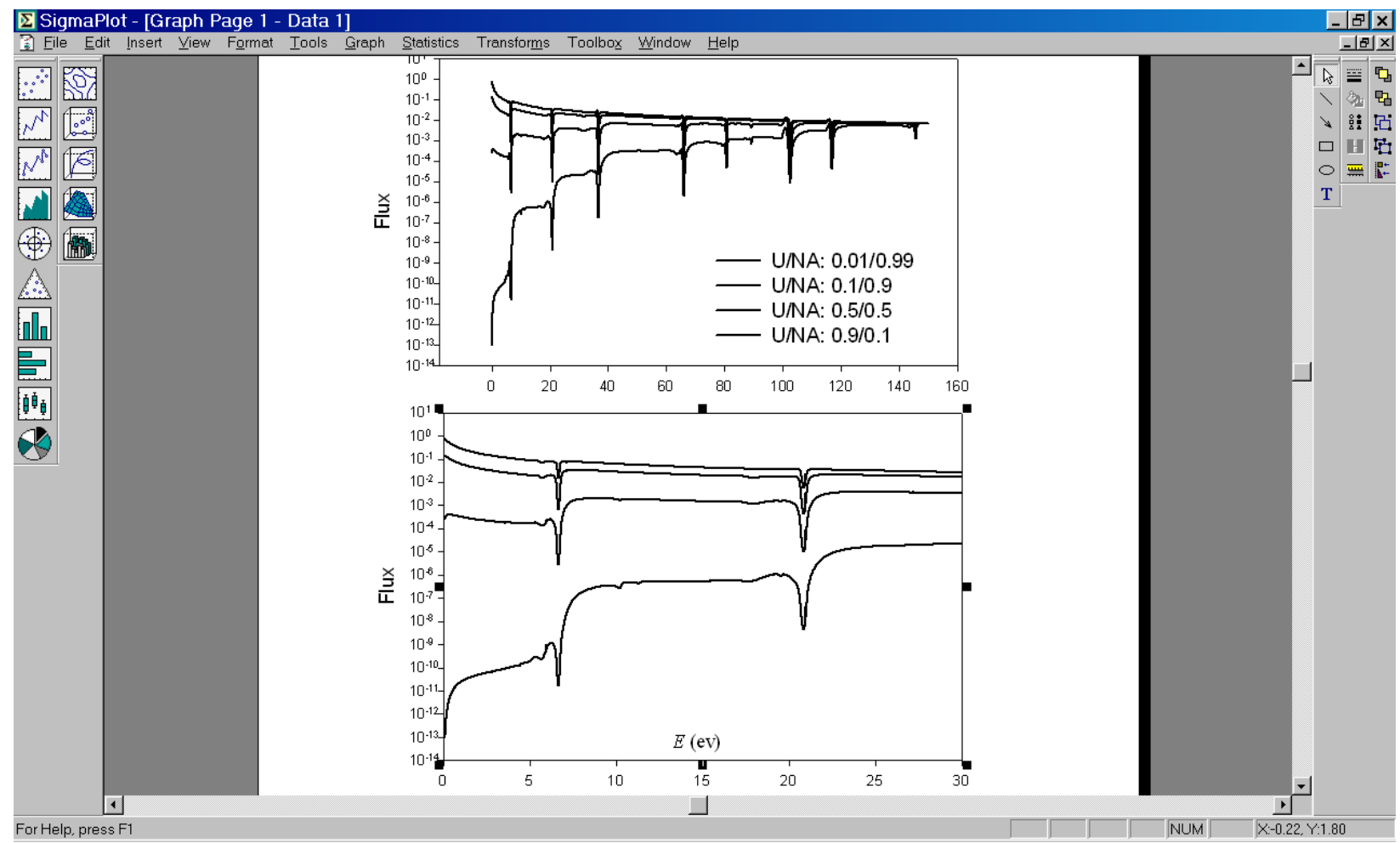

Fig. 4. Flux variation with composition for $G=10$ and $N=30,000$.

Slide 2 shows the results of a timing study to determine an optimal $G$. For the direct inversion, it seems that for a fine-group calculation of a thousand groups, a $G$ of 10 is optimal.

Slides 3 and 4 are a case study of the $6.67 \mathrm{eV}$ Uranium resonance. Here, as shown in Slide 3, the Single Level Breit-Wigner (SLBW) form of the resonance with a $1 / E$ weighting flux is assumed in the analytical construction of the multigroup cross sections. The energy range is from $12 \mathrm{eV}$ to zero fitted by a uniform lethargy grid. For this granularity, the resonance is generally captured with about 800 groups, again indicating the necessity of a FG/UfG grid.

Slide 5a shows the many resonances over the range $2 \mathrm{eV}<E<800 \mathrm{eV}$ with the source at the top energy requiring 7200 groups for adequate coverage.

Slide $5 \mathbf{b}$ gives the relative error for $G$ chosen to be 1 or 100 indicating near machine double precision. 
Slide 6 shows what can be accomplish with $72 \mathrm{~K}$ groups. While for these calculations, the computational effort seems excessive, realize these are our first attempts without code optimization.

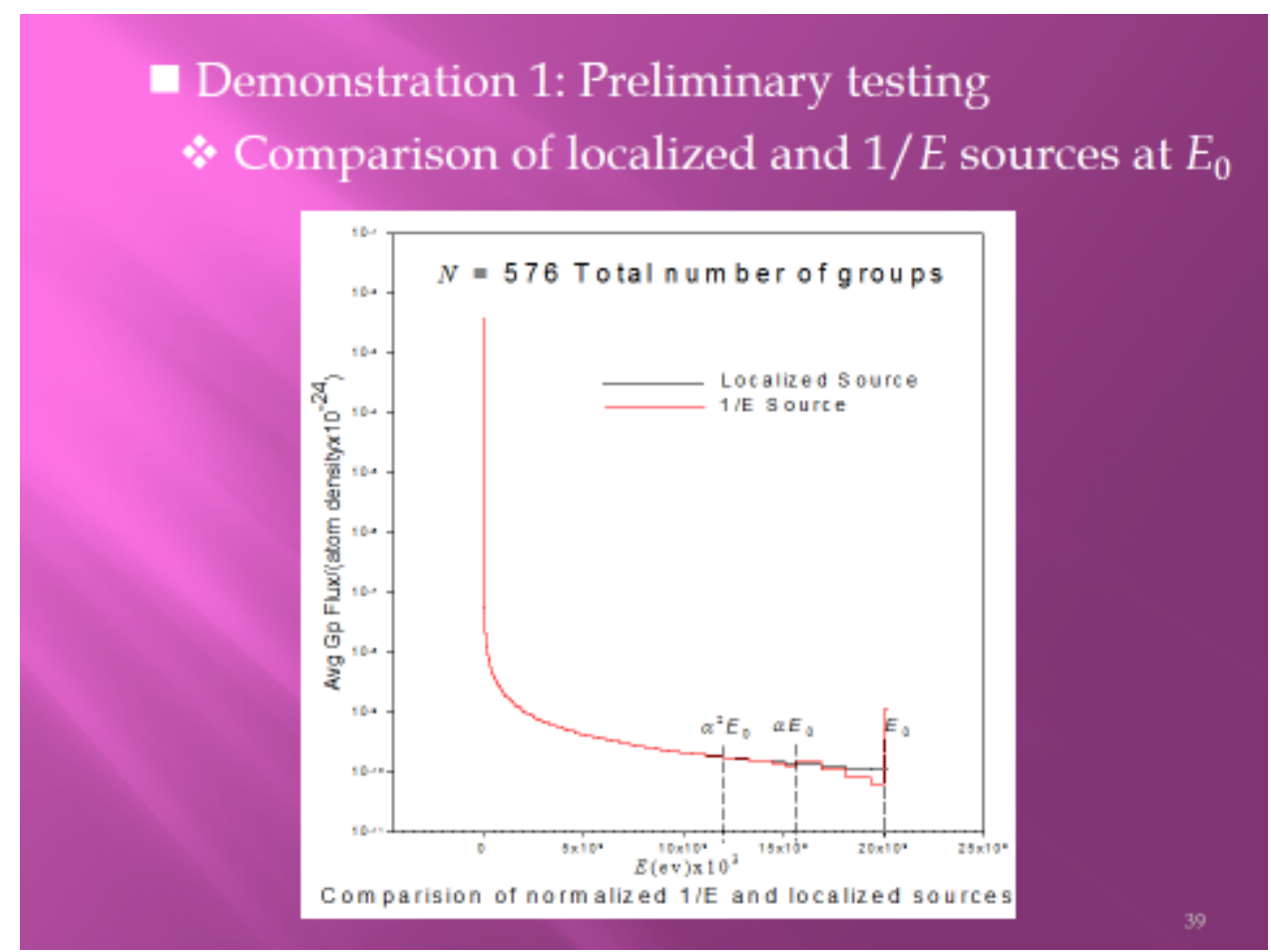

\section{Slide 1}

\section{* What is the advantage of MultiPanel?}

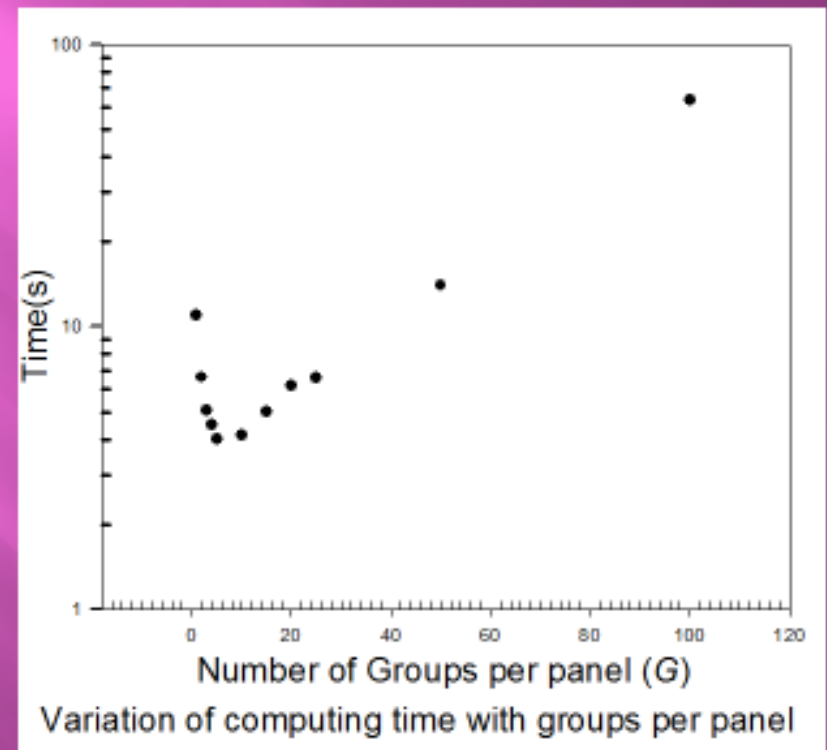

Slide 2 
Demonstration 2: Slowing down in resonances

* For SLBW resonance $(T=0)$

$$
\begin{aligned}
& \sigma_{s}(E)=\frac{\sigma_{r} \Gamma_{n}}{\Gamma} \frac{1}{1+x^{2}}+\frac{2 \sigma_{r} R}{\hbar_{r}} \frac{x}{1+x^{2}}+4 \pi R^{2} \\
& \sigma_{\gamma}(E)=\frac{\sigma_{r} \Gamma_{\gamma}}{\Gamma}\left(\frac{E_{r}}{E}\right)^{1 / 2} \frac{1}{1+x^{2}} \\
& x=\frac{2 \mu}{\Gamma}\left(E-E_{r}\right)
\end{aligned}
$$

* Analytical group data representation for

$$
f(E)=C / E
$$

Slide 3

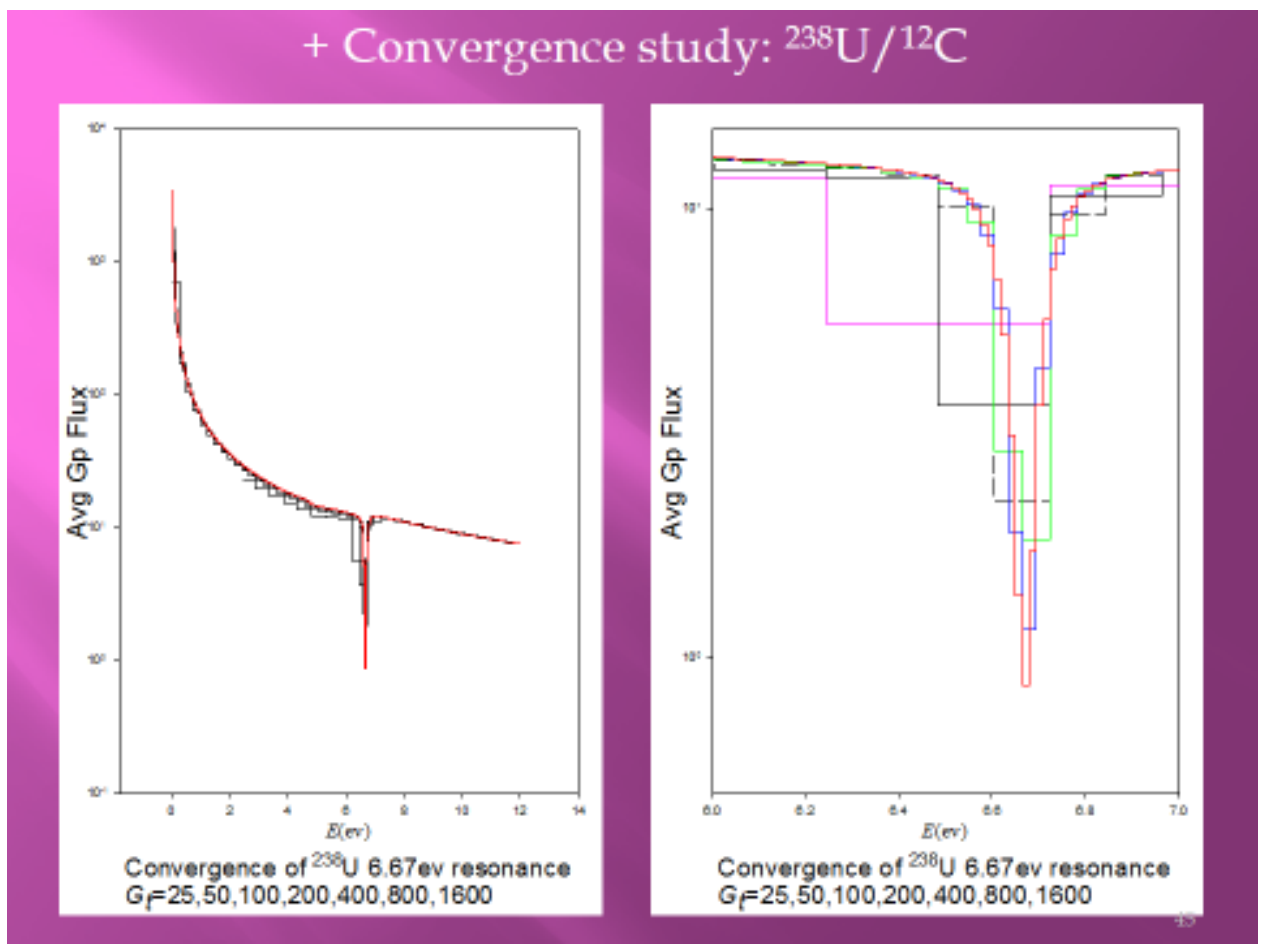

Slide 4 

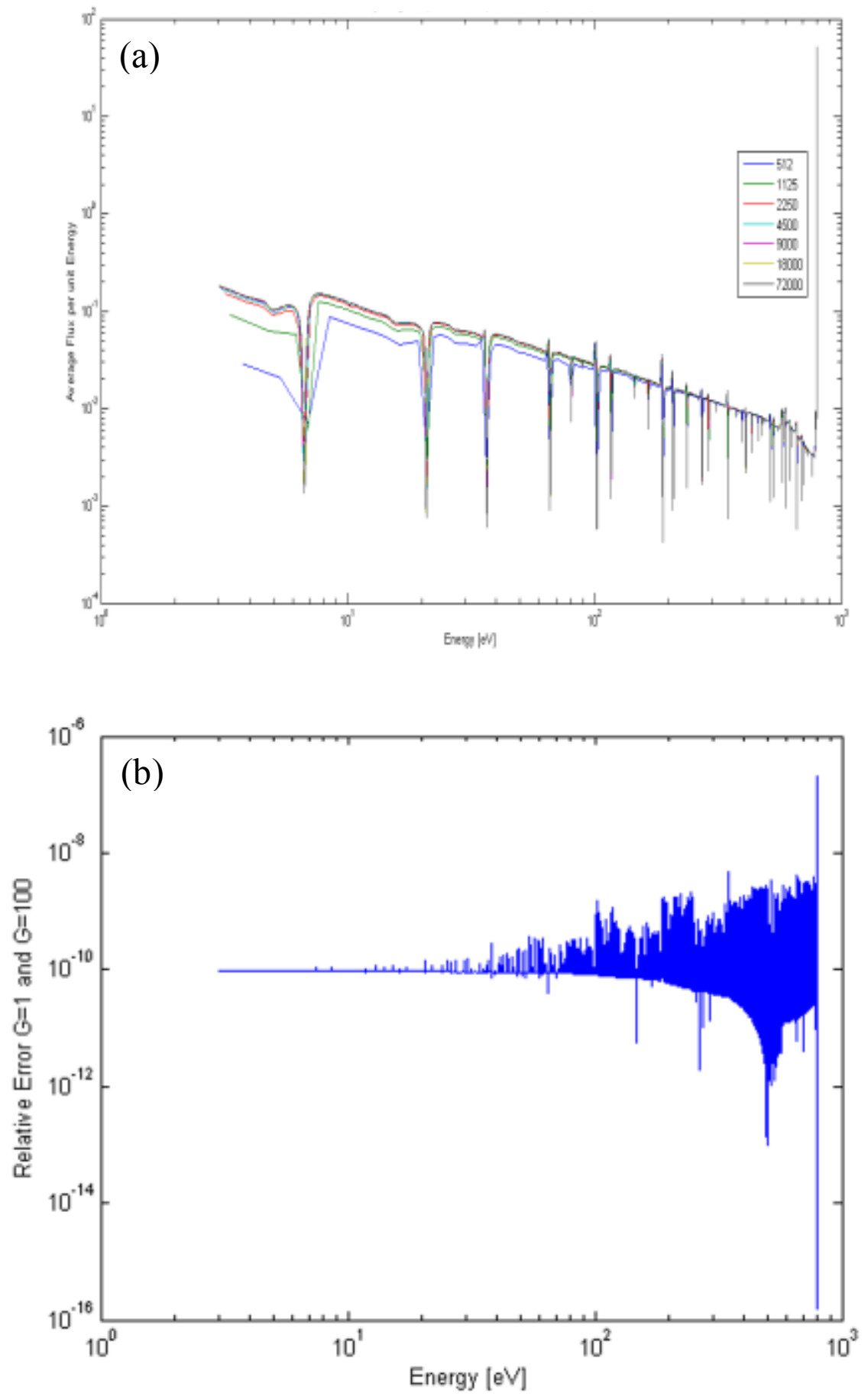

Slides 5a,b 


\section{* 72,000 groups (27 min/1.4 GHz Gateway)}

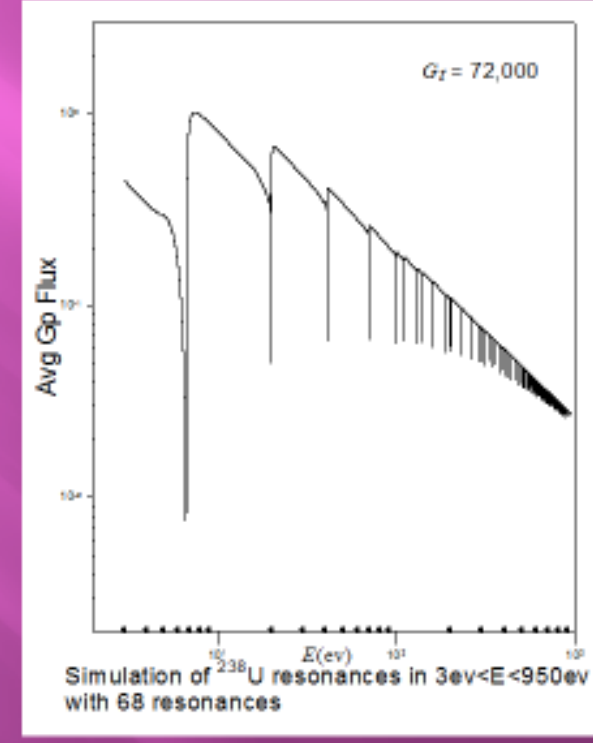

Slide 6

\subsection{Slowing down in the BL approximation}

The infinite medium case just considered represents a special case of the BL approximation. In this approximation, the finiteness of a medium is expressed in terms of a buckling $B_{L}$, but we retain the mathematics of an infinite medium. Direction now must be considered, making the calculation more complex. This work was presented as a part of an INL short course held during the summer of 2013 (A2.MG.BL.Approximation). The derivation of the BL equations follows.

The general slowing down equation in an infinite medium with general scattering and isotropic source is

$$
\left[1+i \alpha_{g} \mu\right] \mathrm{F}_{g}(\mu)=\frac{1}{2} \sum_{l=0}^{L}(2 l+1) P_{l}(\mu) \sum_{g^{\prime}=1}^{G} \omega_{l g g^{\prime}} \mathrm{F}_{l g}+\frac{1}{2} q_{g},
$$

where the group parameters are

$$
\begin{aligned}
& \mathbf{F}(\mu)=\left[\mathrm{F}_{1}(\mu) \mathrm{F}_{2}(\mu) \ldots \mathrm{F}_{G}(\mu)\right]^{T} \\
& \mathbf{F}_{l}=\left[\begin{array}{lll}
\mathrm{F}_{l 1} & \mathrm{~F}_{l 2} \ldots \mathrm{F}_{l G}
\end{array}\right]^{T}, \mathrm{~F}_{l g} \equiv \int_{-1} d \mu P_{l}(\mu) \mathrm{F}_{g}(\mu) \\
& \boldsymbol{q}=\left[\begin{array}{ll}
q_{1} & q_{2} \ldots q_{G}
\end{array}\right]^{T} \\
& \underline{\boldsymbol{\omega}}_{l}=\left[\omega_{l g g^{\prime}}, g, g^{\prime}=1, \ldots, G\right]
\end{aligned}
$$




$$
\underline{\alpha}=\operatorname{diag}\left\{B_{L} / \Sigma_{g} ; g=1, \ldots, G\right\} .
$$

Specifically, $\mathbf{F}(\mu)$ and $\boldsymbol{q}$ are the group collision density vector and the imposed isotropic source strength vector respectively and

$$
\begin{aligned}
& \mathbf{F}_{l} \text { is the Legendre moment: }\left\{\mathrm{F}_{l g} \equiv \int_{-1}^{1} d \mu P_{l}(\mu) \mathrm{F}_{g}(\mu)\right\} \\
& \underline{\omega}_{l} \text { is the transfer scattering cross section coefficient } \\
& B_{L} \text { is the geometric buckling. }
\end{aligned}
$$

Therefore, in fundamental matrix form, we have

$$
[\boldsymbol{I}+i \boldsymbol{\alpha} u] \mathbf{F}(\mu)=\frac{1}{2} \sum_{l=0}^{L}(2 l+1) P_{l}(\mu) \boldsymbol{\omega}_{l} \mathbf{F}_{l}+\frac{1}{2} \boldsymbol{I}
$$

where $\mathbf{F}(\mu)=\boldsymbol{F}(\mu) \boldsymbol{q}$ with $\boldsymbol{F}(\mu)$ expressed as a matrix.

Equation (2) is a Green's function for full slowing down in an infinite medium with scattering order truncated at $L$. This equation was one of the first to be solved to generate representative reactor specific cross sections.

\section{a. First solution}

Simply by projection over the Legendre polynomials, we arrive at a first solution in the form

$$
\sum_{l=0}^{L}\left[\delta_{l j} \boldsymbol{I}-(2 l+1) z \boldsymbol{L}_{j l}(z) \boldsymbol{\omega}_{l}\right] \boldsymbol{F}_{l}(z)=(-1)^{j} z \boldsymbol{Q}_{j}(z),
$$

with

$$
\begin{aligned}
& z=\frac{1}{i B_{L}} \\
& \boldsymbol{L}_{j l}(z) \equiv\left\{L_{j l}\left(z_{g}\right) \equiv \frac{z_{g}}{2} \int_{-1}^{1} d \mu \frac{P_{j}(\mu) P_{l}(\mu)}{\mu-z_{g}} ; j, l=0,1, \ldots, L ; g=1, \ldots, G\right\} \\
& \boldsymbol{Q}_{j}(z) \equiv \operatorname{diag}\left\{Q_{j}\left(z_{g}\right) \equiv \frac{1}{2} \int_{-1}^{1} d \mu \frac{P_{j}(\mu)}{\mu-z_{g}} ; g=1, \ldots, G\right\}
\end{aligned}
$$

\section{b. Second solution}

A second set of moments equations is found by projecting $\operatorname{Eq}(2)$ over the Legendre polynomials $P_{j}(\mu)$ to give for $j$ in $[0, L]$ 


$$
z \boldsymbol{h}_{j} \boldsymbol{F}_{j}(z)+(j+1) \boldsymbol{F}_{j+1}(z)+j \boldsymbol{F}_{j-1}(z)=z \delta_{j 0} \equiv \boldsymbol{f}_{j}(z)
$$

with $\boldsymbol{h}_{j} \equiv(2 j+1)\left(\boldsymbol{I}-\boldsymbol{\omega}_{j}\right)$.

The general solution to the three-point recurrence is completely analogous to the solution to a second order ODE. In particular, the solution consists of two complimentary solutions and a particular solution. The procedure to find the analytical solution is outlined as follows:

1. Express the solution in terms of homogeneous and particular solutions:

2. Substitute into $\mathrm{Eq}(4)$ :

$$
\boldsymbol{F}_{l}(z)=\boldsymbol{g}_{l}(z) \boldsymbol{a}(z)-\rho_{l}(z) \boldsymbol{b}(z)+\sum_{j=1}^{l-1} \boldsymbol{\alpha}_{l j} \boldsymbol{f}_{j}(z) .
$$

$$
\begin{aligned}
{\left[z \boldsymbol{h}_{j} \boldsymbol{g}_{l}(z)+\right.} & \left.(j+1) \boldsymbol{g}_{j+1}(z)+j \boldsymbol{g}_{j-1}(z)\right] \boldsymbol{a}(z)+ \\
+\left[z \boldsymbol{h}_{j} \boldsymbol{\rho}_{l}(z)\right. & \left.+(j+1) \boldsymbol{\rho}_{j+1}(z)+j \boldsymbol{\rho}_{j-1}(z)\right] \boldsymbol{b}(z)+ \\
& +\sum_{j=0}^{l}\left[z \boldsymbol{h}_{j} \boldsymbol{\alpha}_{l, j}(z)+(j+1) \boldsymbol{\alpha}_{l, j+1}(z)+j \boldsymbol{\alpha}_{l, j-1}(z)\right] \boldsymbol{f}_{j}(z)+ \\
& +\left[(l+1) \boldsymbol{\alpha}_{l+1, l+1} \boldsymbol{f}_{l+1}(z)\right]-\left[l \boldsymbol{\alpha}_{l-1, l} \boldsymbol{f}_{l}(z)-\boldsymbol{f}_{l}(z)\right]=0 .
\end{aligned}
$$

3. Set terms in brackets to zero:

$$
\begin{aligned}
& z \boldsymbol{h}_{l} \boldsymbol{g}_{l}(z)+(l+1) \boldsymbol{g}_{l+1}(z)+l \boldsymbol{g}_{l-1}(z)=0 \\
& z \boldsymbol{h}_{l} \boldsymbol{\rho}_{l}(z)+(l+1) \boldsymbol{\rho}_{l+1}(z)+l \boldsymbol{\rho}_{l-1}(z)=-z \delta_{l, 0} \\
& z \boldsymbol{h}_{l} \boldsymbol{\alpha}_{j, l}(z)+(l+1) \boldsymbol{\alpha}_{j, l+1}(z)+l \boldsymbol{\alpha}_{j, l-1}(z)=0 \\
& (l+1) \boldsymbol{\alpha}_{l+1, l+1} \equiv \mathbf{0} \\
& l \boldsymbol{\alpha}_{l-1, l} \equiv \boldsymbol{I} .
\end{aligned}
$$

The inhomogeneous term for the second equation above is assumed to give

$$
\begin{aligned}
& \boldsymbol{a}(z) \equiv \boldsymbol{F}_{0}(z) \\
& \boldsymbol{b}(z) \equiv \boldsymbol{I} .
\end{aligned}
$$

4. The following form for $\boldsymbol{\alpha}_{j, l} l>0$ satisfies the recurrence for $\boldsymbol{\alpha}_{j, l}$ :

$$
\alpha_{j, l}=g_{l}(z) u_{j}(z)+\rho_{l}(z) v_{j}(z)
$$


if

$$
\begin{aligned}
& \mathbf{0}=\boldsymbol{g}_{l}(z) \boldsymbol{u}_{l}(z)+\boldsymbol{\rho}_{l}(z) \boldsymbol{v}_{l}(z) \\
& \boldsymbol{\alpha}_{l-1, l}=\frac{1}{l} \boldsymbol{I}=\boldsymbol{g}_{l-1}(z) \boldsymbol{u}_{l}(z)+\boldsymbol{\rho}_{l-1}(z) \boldsymbol{v}_{l}(z),
\end{aligned}
$$

to give

$$
\begin{aligned}
& \boldsymbol{v}_{l}(z)=-\frac{1}{l} \boldsymbol{\rho}_{l}^{-1}(z)\left[\boldsymbol{\rho}_{l-1}(z) \boldsymbol{\rho}_{l}^{-1}(\boldsymbol{z})-\boldsymbol{g}_{l-1}(z) \boldsymbol{g}_{l}^{-1}(z)\right]^{-1} \\
& \boldsymbol{u}_{l}(z)=\frac{1}{l} \boldsymbol{g}_{l}^{-1}(z)\left[\boldsymbol{\rho}_{l-1}(z) \boldsymbol{\rho}_{l}^{-1}(z)-\boldsymbol{g}_{l-1}(z) \boldsymbol{g}_{l}^{-1}(z)\right]^{-1}
\end{aligned}
$$

5. The final expression:

with

$$
\begin{aligned}
\boldsymbol{F}_{l}(z) & =\boldsymbol{g}_{l}(z) \boldsymbol{F}_{0}(z)-\rho_{l}(z)+ \\
& +\sum_{j=1}^{l-1}\left[\boldsymbol{g}_{l}(z) \boldsymbol{g}_{j}^{-1}(z)-\boldsymbol{\rho}_{l}(z) \boldsymbol{\rho}_{j}^{-1}(z)\right] W_{j}^{-1}(z) \boldsymbol{f}_{j}(z)
\end{aligned}
$$

$$
W_{j}(z) \equiv \frac{1}{j}\left[\boldsymbol{\rho}_{j-1}(z) \boldsymbol{\rho}_{j}^{-1}(z)-\boldsymbol{g}_{j-1}(z) \boldsymbol{g}_{j}^{-1}(z)\right]^{-1} .
$$

Now introduce $\mathrm{Eq}(5)$ into $\mathrm{Eq}(3)$ to give the scalar collision density

$$
\begin{aligned}
\boldsymbol{F}_{0}(z)= & {\left[I-\sum_{l=0}^{L}(2 l+1) z \boldsymbol{Q}_{l}(z) \boldsymbol{\omega}_{l} \boldsymbol{g}_{l}(z)\right]^{-1} \times } \\
& \times\left[z \boldsymbol{Q}_{0}(z)-\sum_{l=0}^{L}(2 l+1) z \boldsymbol{Q}_{l}(z) \boldsymbol{\omega}_{l} \boldsymbol{\rho}_{l}(z)\right],
\end{aligned}
$$

which (with some algebra) can be shown to be

$$
\begin{aligned}
& \boldsymbol{F}_{0}(z)= \\
& =\left[\boldsymbol{Q}_{L}(z) g \varrho_{L+1}(z)-\boldsymbol{Q}_{L+1}(z) g(z)\right]^{-1}\left[\boldsymbol{Q}_{L}(z) \not Q_{L+1}(z)-\boldsymbol{Q}_{L+1}(z) \not \boldsymbol{\beta}_{L}(z)\right]
\end{aligned}
$$

where $f_{l}^{g}\left(\boldsymbol{q}_{z}\right)=(-1)^{l} \boldsymbol{f}_{l}(z)$ with $\boldsymbol{f}_{l}^{\prime /}$ either $\boldsymbol{g}_{l}$ or $\rho_{l}$ and $\boldsymbol{Q}_{l}$ is a diagonal matrix of Legendre functions of the second kind.

A monogenetic form of the above was presented at ICTT23 (A3.ICTT23). 
Coding of this result will be completed in a future effort. As a first example however, the following table represents an initial attempt presented at the Math and Comp 2013 Benchmarking Workshop (A4.MC13.Wkshp).

\section{Zeroth Moment $F_{0 L}$ for Helium: $L=[0(1) 6], B=1$

$$
\text { Convergence in scattering order } / \varepsilon=10^{-\dagger}
$$

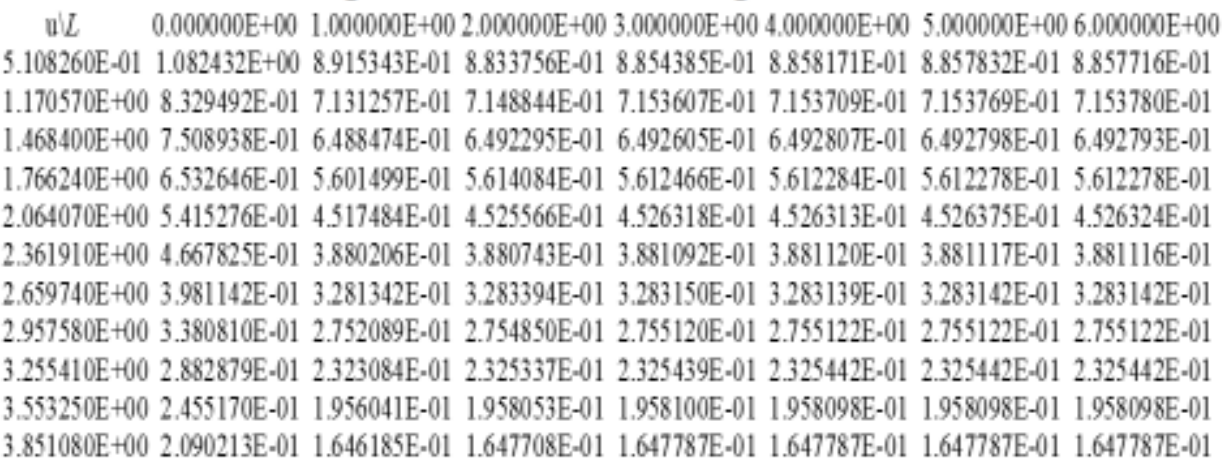

Table 1. Zeroth moment for Helium with increasing anisotropy to $L=6$.

Table 1 gives the zeroth flux moment variation in lethargy $u$ for increasing anisotropy $(L)$ in Helium for the constant cross section approximation and $B_{L}=$ 1 , corresponding to a reactor on the order of width $\pi$. Note that as lethargy increases the scalar flux become less dependent on the anisotropy.

This is one of the first true analytical solutions to the $B_{L}$ equations for isotropic source emission and represents an advance in the numerical determination of flux moments.

\subsection{Generation of ENDF/B cross section data: Automated}

Using the PYTHON scripting language, the cross section data transfer from BNL/NNDC was automated and the following describes the process. This work was presented at ICTT23 held in Santa Fe, NM/9.15.13 and was completed by the UfG software team (Dr. I. Guven and M. Futch). The PPT presentation is reproduced here.

The software tool consists of three parts

Data Retrieval

Analysis

Review of Results 
arranged as shown in Slide 1. The process is essentially the same as in §II.1.2,

\section{The Software Tool}

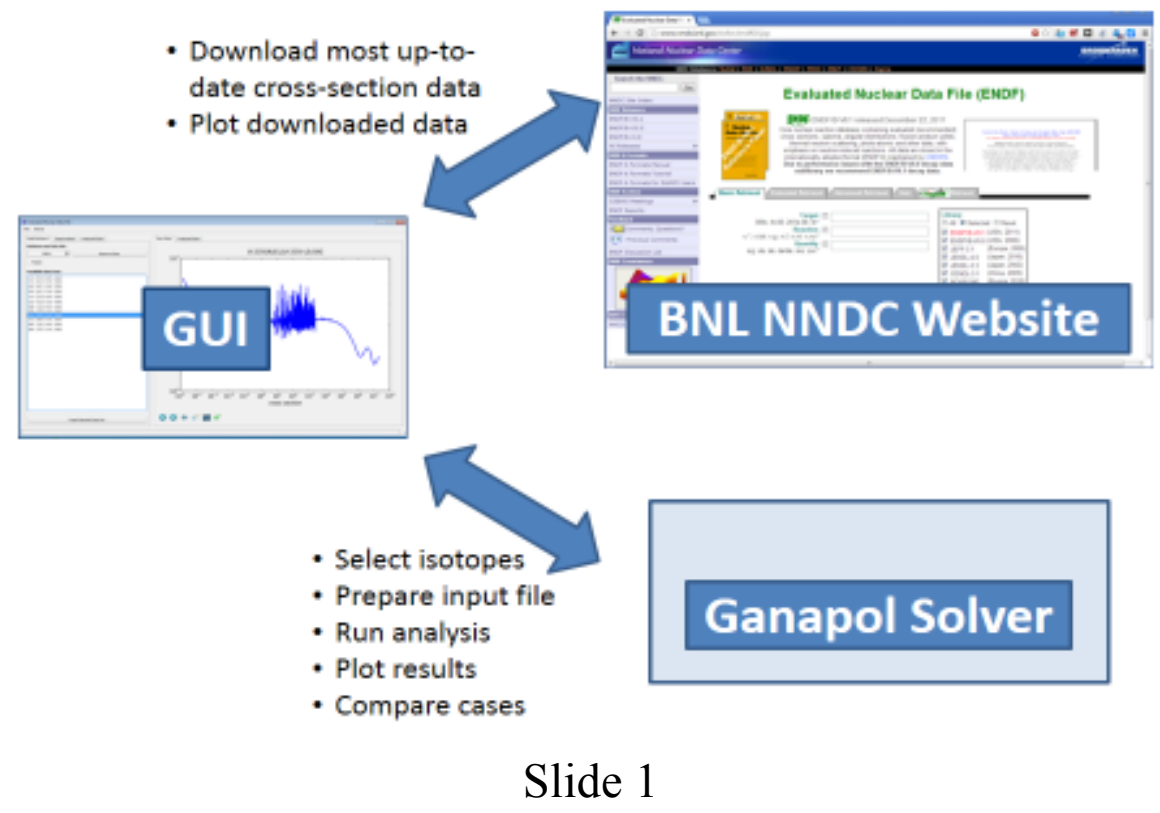

where isotopes are selected and brought locally, processed by the UfG processor $\mathbf{X} 2$ and the flux plotted.

\section{Data Retrieval}

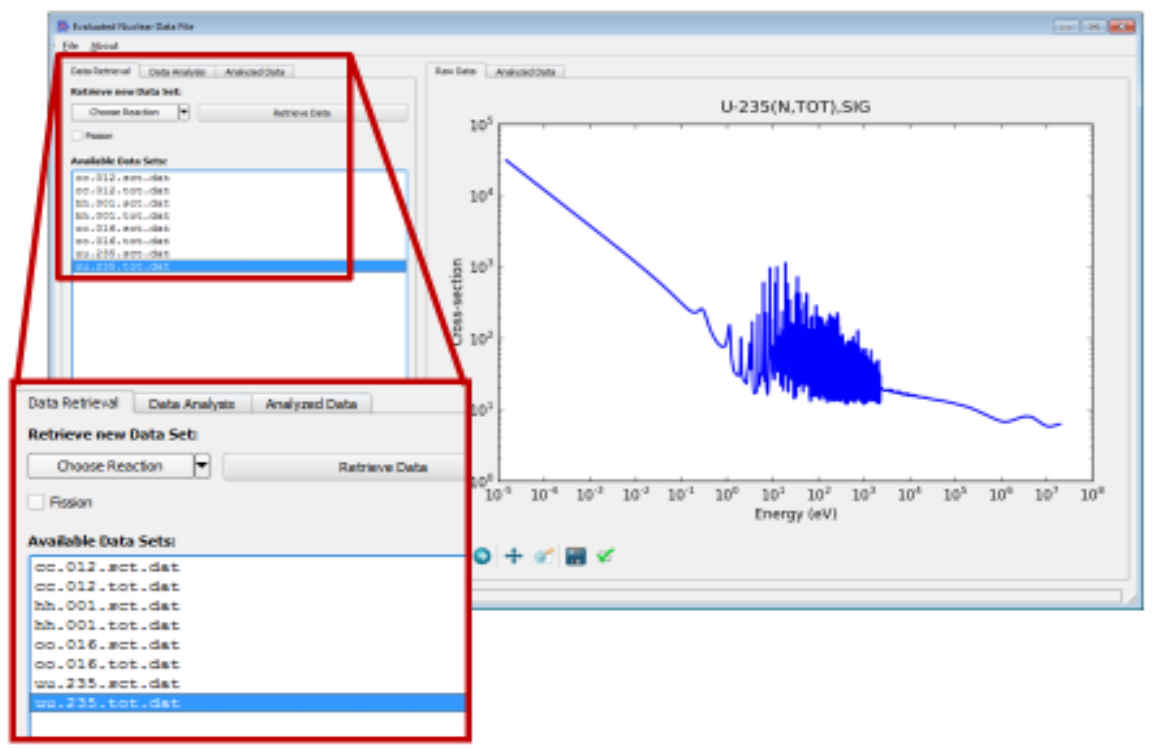

Slide 2 
To begin, "Data Retrieval" (Slide 2) is selected and by selecting "choose element" nuclides are selected from the list that appears as shown in Slide 3. An element

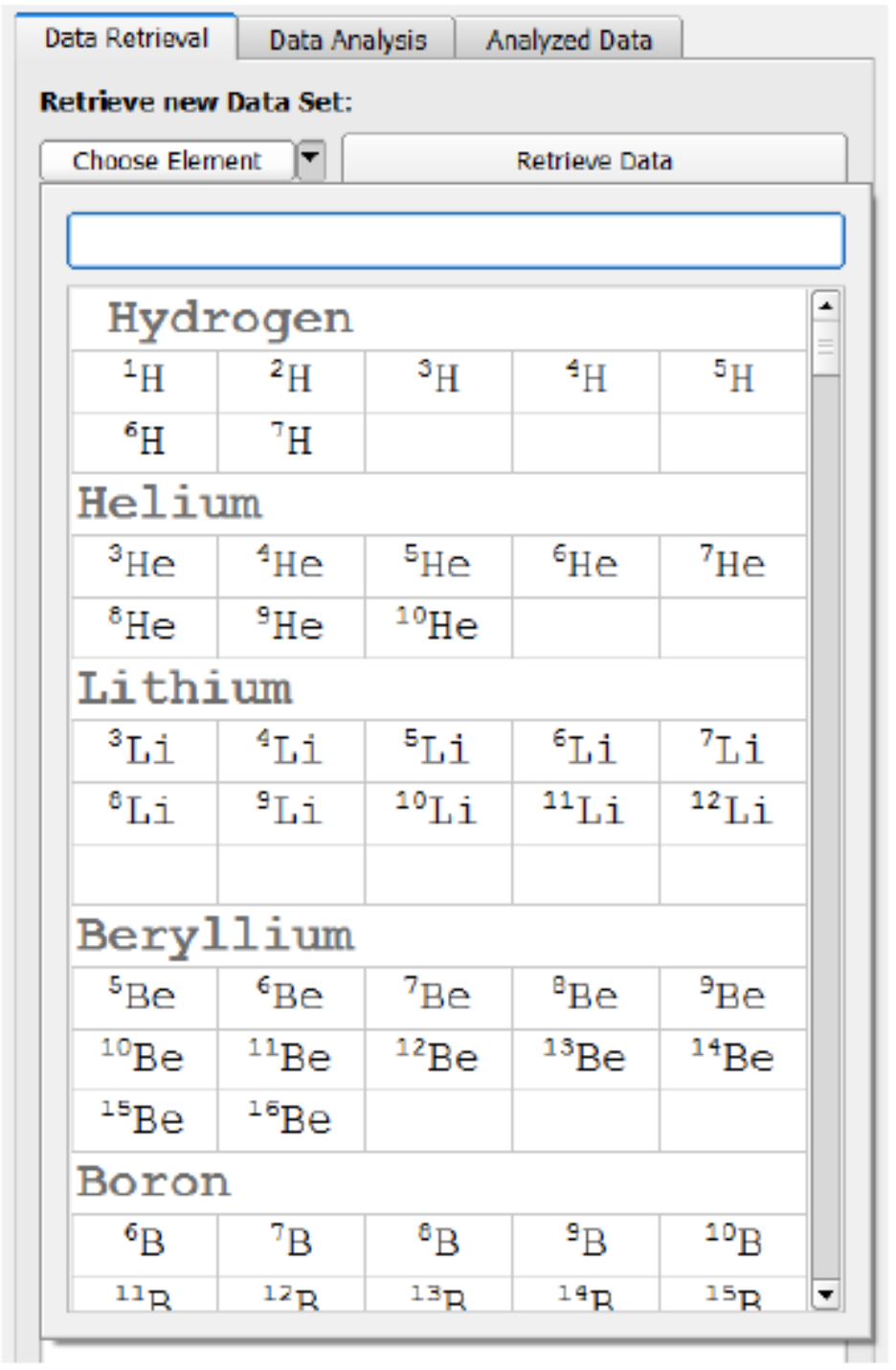

Slide 3

is chosen and total and scattering microscopic cross sections are transferred by selecting "Retrieve Data" (Slide 2). A list of the retrieved data is generated and one can plot individual cross sections if desired by selecting "Plot Data" and a desired entry (Slide 2) on the list. Next, selecting "Data Analysis", a cumulative list of the available nuclides appears for mixing as shown in Slide 4. Those to be mixed according to a specified volume fraction are selected and a volume fraction entered. When mixing is complete, "Analyze" is selected to run the X2 
slowing down UfG processor with the selected data and plot the flux also shown in Slide 4. The results are stored and can be recalled later for plotting as shown in Slide 5, which shows a U235/Carbon slowing down spectrum.

A manual and movie for the installation and operation of the X2/UfG processor GUI are available upon request (GUI, The Manual and GUI, The Movie)

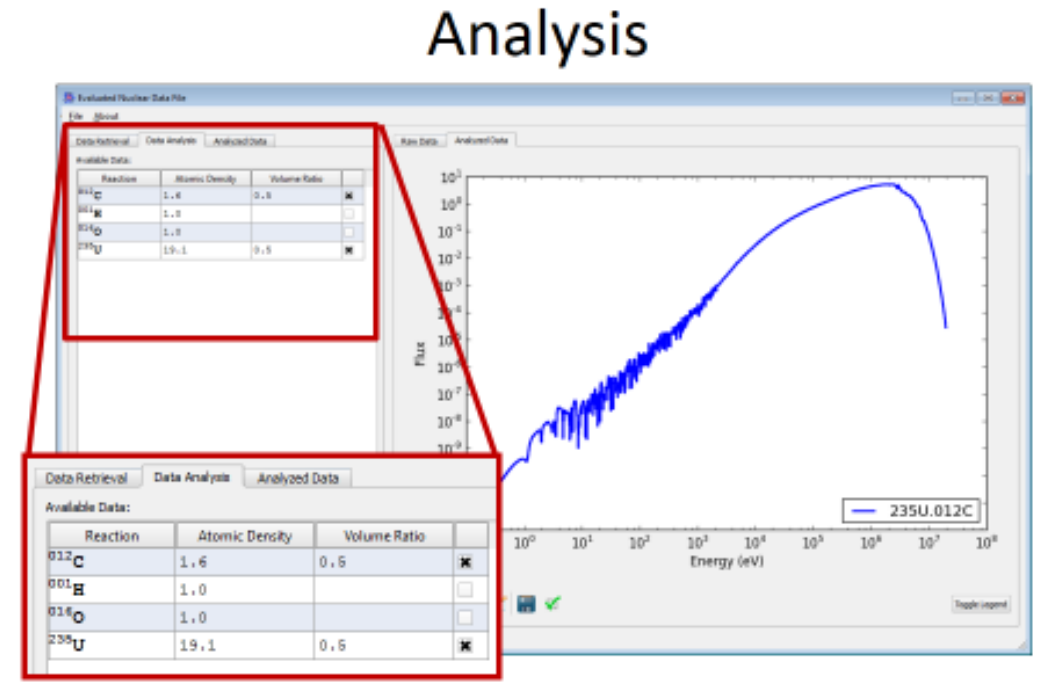

Slide 4

Review Results

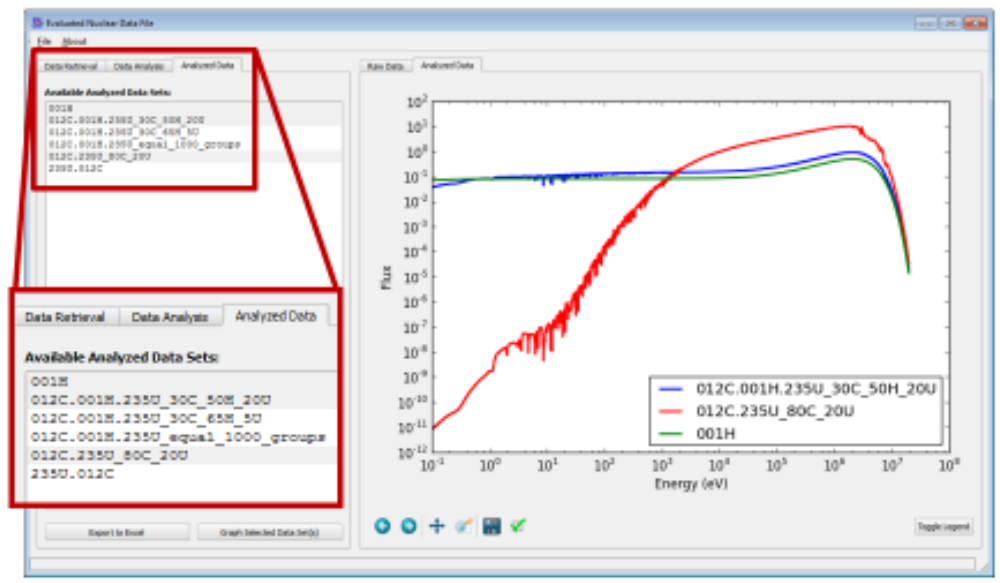

Slide 5 
II.2. TASK 2 Develop the panel algorithm for multimaterials.

While the analysis thus far has passively included multimaterials, there has been no special considerations for their inclusion. During the third year of the grant, visiting student scholar Nicholas Terranova considered how to choose the group structure with regard to multimaterials. In his ANS summary, A5.Adaptive.Mg, he presents a method for a more accurate choice of the multigroup structure for multimaterials. In all previous calculations and demonstrations, a uniform lethargy grid has been assumed, which is most convenient but completely ignores the variation of the cross sections with energy. Also, the uniform grid applies to all materials alike. A more sensible approach is to use the data variation from the ENDF database itself to capture the variation. In addressing Task 1, it was noted that each material for each interaction has cross section data at different energy entries. For a uniform in lethargy grid, this requires interpolation to the chosen grid, again ignoring all variation. If, instead, one grid, based on the cumulative set of all ENDF entries for each material of interest is constructed, then all variation, to the extent it is has been recorded in ENDF/B files, is now addressed. However, this would require millions of groups and would not be feasible. A first step in reconciling this issue would be to perform a thinning process that reduces the total number of energy points to a reasonable number. In this task, we address the overall efficiency of using the uniform grid versus a non-uniform grid based on the actual data entries restricted to a limited number of groups. The procedure that was developed is called Natural MG (NMG).

\subsection{Theory of the NMG approximation}

The theory of the NMG will be presented through consideration of the following slowing down equation in an infinite medium:

$$
\Sigma(E) \phi(E)=\sum_{g^{\prime}=1}^{G} \int_{\Delta E_{g^{\prime}}} d E \Sigma_{s f}\left(E^{\prime} \rightarrow E\right) \phi\left(E^{\prime}\right)+Q(E),
$$

where the scattering and fission terms have been combined and the integration over the energy spectrum partitioned into $G$ energy groups. When we integrate $\mathrm{Eq}(1)$ over the group $g$ and use the integral mean value theorem to remove the flux from the integral, we find the NMG equation 


$$
\begin{aligned}
& \int_{\Delta E_{g}} d E \Sigma(E) \phi(E)= \\
& =\sum_{g^{\prime}=1}^{G} \int_{\Delta E_{g}} d E \int_{\Delta E_{g^{\prime}}} d E^{\prime} \Sigma\left(E^{\prime} \rightarrow E\right) \phi\left(E^{\prime}\right)+\int_{\Delta E_{g}} d E \Sigma(E) Q(E)
\end{aligned}
$$

or in the usual multigroup form

$$
\Sigma_{g} \phi_{g}=\sum_{g^{\prime}=1}^{G} \Sigma_{g g^{\prime}} \phi_{g^{\prime}}+Q_{g}, g=1, \ldots, G
$$

if we define

$$
\begin{aligned}
& \left\{\phi_{g}, Q_{g}\right\} \equiv\left\{\phi\left(E_{g}^{0}\right) \Delta E_{g}, Q\left(E_{g}^{0}\right) \Delta E_{g}\right\} \\
& \Sigma_{g} \equiv \frac{1}{\Delta E_{g}} \int_{\Delta E_{g}} d E \Sigma_{g}(E) \\
& \Sigma_{g g^{\prime}} \equiv \frac{1}{\Delta E_{g^{\prime}}} \int_{\Delta E_{g}} d E \int_{\Delta E_{g^{\prime}}} d E \Sigma_{s f}\left(E^{\prime} \rightarrow E\right) .
\end{aligned}
$$

It should be emphasized that $\mathrm{Eq}(2)$ is exact if the $\mathrm{E}_{g}^{0}$ were known. Also, note that there is no weighting flux to be guessed if it is known. This feature requires further investigation however.

We now analyze the total cross section in the NMG approximation.

A common practice in forming the energy groups $\Delta E_{g}$ is to assume groups uniform in lethargy. While certainly convenient, such a procedure entirely ignores the cross section variation and leads to missing data. This can be especially catastrophic with regard to resonances. For this reason, the NMG follows a more natural and evident path.

To achieve a non-uniform group structure able to better match the data (from the ENDF database) and provide dense coverage near and within resonances, a smart way to construct the groups is to employ the information we already have in the database. For example, one could consider the spectrum as a sequence of edit points, as given to us from the ENDF database. Rather than associate the 
same width to each energy group, we assign the same number of edit points from the original energy structure to the sequence. The right boundary of a group will be midway between the edit points $N$ and $N+1$, where $N$ is the number of edit points per group. The left boundary will obviously coincide with the previous group's right boundary. For the first group, the left boundary will be the minimum of the energy range selected. In this way, because of the ENDF data grouping, next to the resonance, where there will be a high density of values of retrieved cross sections, we will automatically have smaller groups. Since dividing the number of edit points available by the number of groups gives generally a reminder, we can associate these extra points to the last groups considered-- one per group.

\subsection{Numerical demonstration}

A FORTRAN program able to exercise the NMG was written. The code considers three different materials $(\mathrm{U}, \mathrm{O}, \mathrm{H})$. Since in the ENDF database each element has its own energy structure, before implementing our multigroup choice, an interpolation to the same energy boundaries for all materials was performed. We now consider two ranges of energy in order to show the potential of this simple approach to generate a group structure.

In Figs. 1a,b, the NMG group total cross section for U238 has been plotted for energy ranges of $3 \mathrm{eV}-8 \mathrm{eV}$ and $1000 \mathrm{eV}-2200 \mathrm{eV}$. The former shows the group structure regarding a single resonance of the U238; the latter instead shows a series of resonances. By increasing the numbers of groups, it is clear the group cross sections converge to the data values enabling resolution of a significant number of resonances with only a modest number of groups $(<200)$.

In Figs. 2a and 2b, a comparison between the NMG and a uniform lethargy subdivision structure is shown for the two ranges of energy considered. The former is for a 50-group calculation in the $3 \mathrm{eV}-8 \mathrm{eV}$ range, the latter a 200-group calculation for $1000 \mathrm{eV}-2200 \mathrm{eV}$. Both figures show how much the NMG can be more precise than the conventional uniform method. For the range of $3-8 \mathrm{eV}$, in the NMG method, $72 \%$ of the groups reside under the single resonance at $6.67 \mathrm{eV}$, compared to the $20 \%$ for the uniform lethargy grid. In addition, the resonance maxima are more faithfully reproduced with NMG. Thus, the NMG can get more detail from the original nuclear data by increasing the number of groups under the resonances.

Some limitations of the NMG were noted however. For very wide ranges of energies (e.g., $1 \mathrm{eV}-10,000 \mathrm{eV}$ ), including many adjacent resonances, and for a 
relatively small number of groups (e.g. 200), some isolated resonances can be lost, even if the method still shows their position. In fact, the NMG is inclined to concentrate the groups available in the regions of the spectrum with the high density of adjacent resonances. This leads to lower precision where the resonance
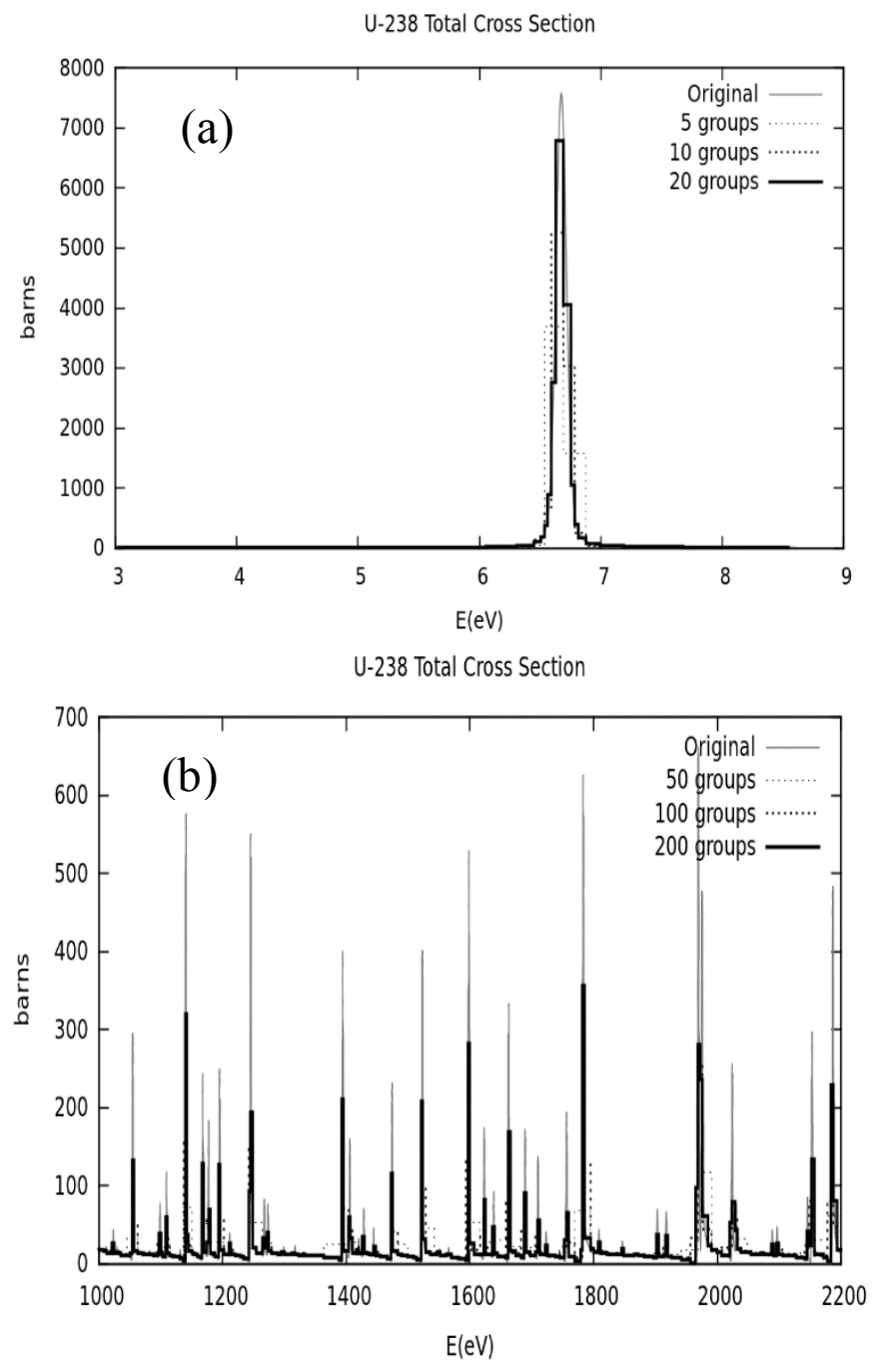

Fig.1. Variation of the number of groups for (a) single and (b) multiple resonances 

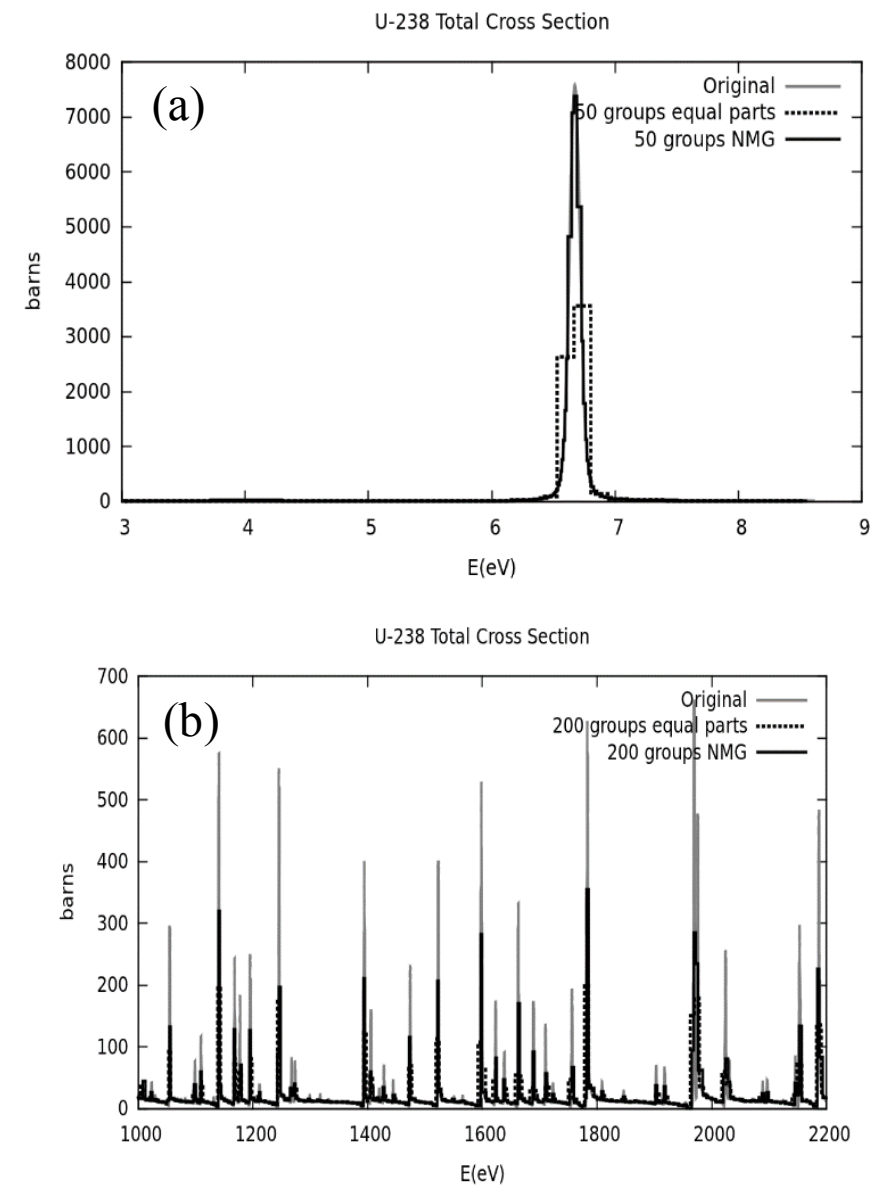

Figs.2. Comparison between the conventional and NMG methods for (a) $G=50$ calculation in $3 \mathrm{eV}-8 \mathrm{eV}$ range. (b) $G=200$ group calculation in range $1000 \mathrm{eV}-2200 \mathrm{eV}$.

density is not large. So if we have ranges of the spectrum with clear differences in resonance densities, the regions with fewer resonances will be penalized. Therefore, a coarse subdivision of the spectrum is desirable before applying the NMG method in order to provide a sufficient number of groups to sense the details

of each resonance.

The NMG method requires further clarification before it can be widely implemented. For this reason, and lack of time, it will possibly become a part of a future effort. After its development, it become known to the PI that this method is actually implemented in the NJOY cross section data processing package [1]. 
II.3. TASK 3 Establish a multigroup/fine-group converged 1D transport and diffusion accelerated algorithm in the panel formalism.

Before realizing full multipanel implementation, one must have an operational single panel option upon which to base the multipanel formulation. For this reason in this task, we primarily considered spatial approximations in the multigroup format for both transport and diffusion and in MATLAB and FORTRAN implementations.

\subsection{Diffusion theory}

The first approach considered by the PI and Elia Battistini, while a visiting scholar from the University of Bologna, was the analytical solution to the diffusion equation. In addition, Elia performed an initial investigation into the corresponding finite difference (FD) solution. These are programmed in MATLAB. With a second visiting scholar, Nicholas Terranova, also from the University of Bologna, an equivalent FORTRAN approach was followed.

\section{a. Analytical/Numerical diffusion theory: MATLAB implementation a1. Analytical solution}

A6.Analytic M\&C MG Diffusion Paper and A7.Analytic M\&C MG Presentation, A8.EB.thesis

The multigroup diffusion equation to be solved is

$$
\left[\boldsymbol{D}_{j} \frac{d^{2}}{d x^{2}}-\boldsymbol{\Sigma}_{j}\right] \boldsymbol{\phi}_{j}(x)+\left[\boldsymbol{\chi} \boldsymbol{v} \boldsymbol{\Sigma}_{f j}^{T}+\boldsymbol{\Sigma}_{s j}\right] \boldsymbol{\phi}_{j}(x)=-\boldsymbol{Q}_{j}(x)
$$

or

$$
\left[\boldsymbol{I} \frac{d^{2}}{d x^{2}}+\boldsymbol{B}_{j}^{2}\right] \boldsymbol{\phi}_{j}(x)=-\boldsymbol{D}_{j}^{-1} \boldsymbol{Q}_{j}(x)
$$

with

$$
\boldsymbol{B}_{j}^{2}=\boldsymbol{D}_{j}^{-1}\left[\boldsymbol{\chi} \boldsymbol{v} \boldsymbol{\Sigma}_{f j}^{T}-\left(\boldsymbol{\Sigma}_{j}-\boldsymbol{\Sigma}_{s j}\right)\right]
$$

Subsequently, diagonalizing the resulting the $G$ by $G$ interaction matrix gives 


$$
\boldsymbol{B}_{j}^{2} \equiv \mathbf{Z}_{j} \boldsymbol{\Omega}_{j} \mathbf{Z}_{j}^{-1}
$$

and for $\mathrm{Eq}(3 \mathrm{~b})$

$$
\left[\boldsymbol{I} \frac{d^{2}}{d x^{2}}+\boldsymbol{\Omega}_{j}\right] \mathbf{Z}_{j}^{-1} \boldsymbol{\Phi}_{j}(x)=-\mathbf{Z}_{j}^{-1} \boldsymbol{D}_{j}^{-1} \boldsymbol{Q}_{j}(x)
$$

where $\boldsymbol{\Omega}_{j}$ is a diagonal matrix of eigenvalues. The solution, expressed as homogeneous and particular parts, is

$$
\begin{aligned}
\boldsymbol{\phi}_{j}(x)= & \boldsymbol{\alpha}_{j} \boldsymbol{h}_{j}^{+}(x) \boldsymbol{\alpha}_{j}^{-1}\left[\boldsymbol{\phi}_{j}\left(x_{j}\right)-\boldsymbol{\phi}_{P j}\right]+ \\
& +\boldsymbol{\alpha}_{j} \boldsymbol{h}_{j}^{-}(x) \boldsymbol{\alpha}_{j}^{-1}\left[\boldsymbol{\phi}_{j}\left(x_{j-1}\right)-\boldsymbol{\phi}_{P j}\right]+\boldsymbol{\phi}_{P j}
\end{aligned}
$$

for a regionally uniform source. $\boldsymbol{h}_{j}^{ \pm}$is a diagonal matrix of homogeneous solutions

$$
\boldsymbol{h}_{j}^{ \pm}(x) \equiv \operatorname{diag}\left\{h_{j k}^{ \pm}(x) ; k=1, \ldots, G\right\}
$$

where, for plane geometry

$$
h_{j k}^{ \pm}(x)=\left\{\begin{array}{l}
{\left[\frac{\sin \left(\Omega_{j k}\left(x-x_{j-1}\right)\right)}{\sin \left(\Omega_{j k} \Delta_{j}\right)}\right]} \\
{\left[\frac{\sin \left(\Omega_{j k}\left(x_{j}-x\right)\right)}{\sin \left(\Omega_{j k} \Delta_{j}\right)}\right]}
\end{array} .\right.
$$

Note that $\boldsymbol{\alpha}_{j} \boldsymbol{h}_{j}^{+}(x) \boldsymbol{\alpha}_{j}^{-1}$ is what is referred to as a matrix function. There are several ways of evaluating this quantity and matrix diagonalization is one possibility.

Similar solutions can be found in Refs. [1] and [2]. 


\section{a1.1 Application of MATLAB version}

Before he applied his spatial diffusion MATLAB version, Elia determined the flux for pure neutron slowing down in an infinite medium of U238 with 300,000 groups shown in Fig. 1. The flux for the entire resolved resonance region is captured requiring less than 6 minutes on a $2.53 \mathrm{GHz}$ Intel RCoreTM2 Duo T9400, 4Gb RAM, using MATLAB 32 bit version 2009b platform. The detail is striking.

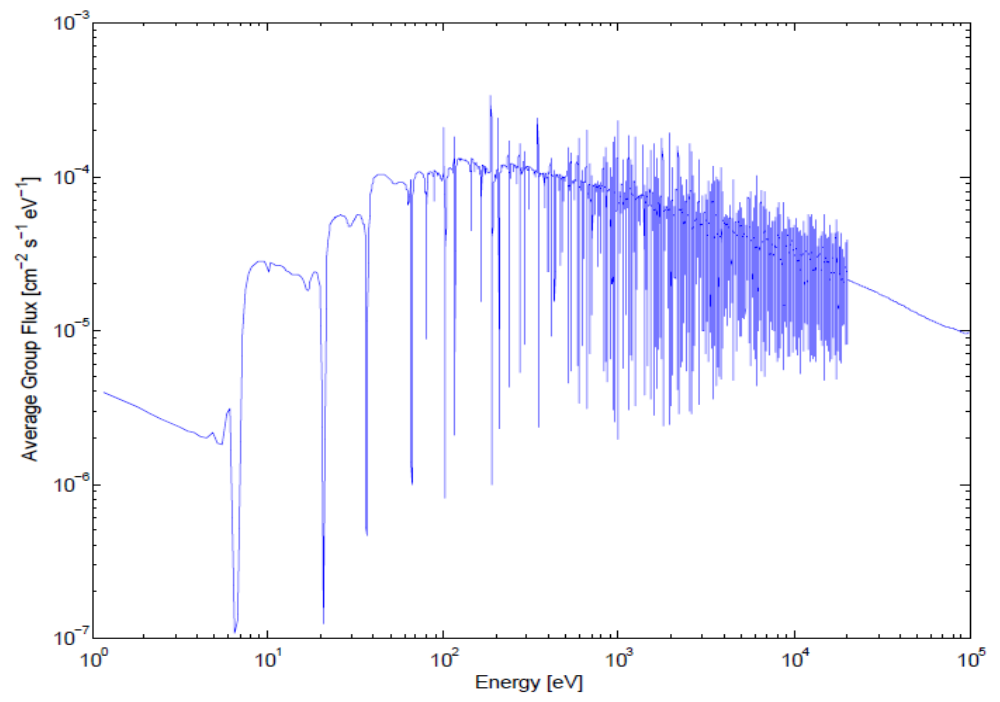

Figure 1 ultra-fine flux calculation involving the whole resolved resonance region of ${ }^{238} \mathrm{U}$.

\begin{tabular}{cccc}
\hline$m$ & Name & Compound & $\begin{array}{c}\text { Thickness } \\
{[\mathrm{cm}]}\end{array}$ \\
\hline 1 & $\mathrm{~F}$ & $\mathrm{UO}_{2}$ & 5 \\
2 & $\mathrm{~W}$ & $\mathrm{H}_{2} \mathrm{O}$ & 15 \\
\hline
\end{tabular}

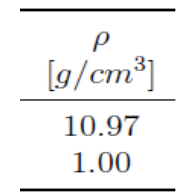

Nuclear composition of the substances the slabs are made of: for $\mathrm{UO}_{2}$ two sets of compositions are given, depending on the presence of the isotope 235 of uranium.

\begin{tabular}{ccccccc}
\hline \multicolumn{3}{c}{$\mathrm{UO}_{2}$} & \multicolumn{3}{c}{$\mathrm{H}_{2} \mathrm{O}$} \\
\hline species & $A$ & $\% w_{1}$ & $\% w_{2}$ & species & $A$ & $\% w$ \\
\hline${ }^{238} \mathrm{U}$ & 238 & 0.865 & 0.881 & ${ }^{1} \mathrm{H}$ & 1 & 0.111 \\
${ }^{235} \mathrm{U}$ & 235 & 0.020 & 0.000 & ${ }^{16} \mathrm{O}$ & 16 & 0.889 \\
${ }^{16} \mathrm{O}$ & 16 & 0.115 & 0.119 & & & \\
\hline
\end{tabular}

Table 1a,b. Medium properties for first test case. 
As a first application of spatial diffusion, a symmetric three-region reactor is assumed with a central depleted $\mathrm{UO}_{2}$ region and water reflectors. A sinusoidal fixed source emits in the central region in each group. The energy range is $[1 \mathrm{eV}, 1000 \mathrm{eV}]$ covered by 10 groups and the slab configuration properties are given in Tables $1 \mathrm{a}, \mathrm{b}$.

Figure 2 shows the results of a benchmark application with the finite difference (FD) solution described in the next section. The FD calculation is run for consecutively reduced mesh discretizations by a factor of 2 . The blue curve gives the groupwise mean relative error over 29 spatial points with respect to the analytical solution for decreasing discretization (increasing number of cells). Note how the error steadily decreases until $10^{5}$ cells and then abruptly increases because of roundoff error. The slope of the curve is two indicating that the FD scheme is of order 2. The other curves represent accelerations of the FD solution to zero discretization as explained in the next section. Observe, all accelerations indeed greatly accelerate convergence to nearly $10^{-12}$; whereas, the FD gives only a $10^{-4}$ relative error.

The above comparison was performed for only 10 groups. If pushed to higher than 20 groups one encounters stability issues with regard to the diagonalization of the interaction matrix. This negative result may indicate that the analytical diffusion

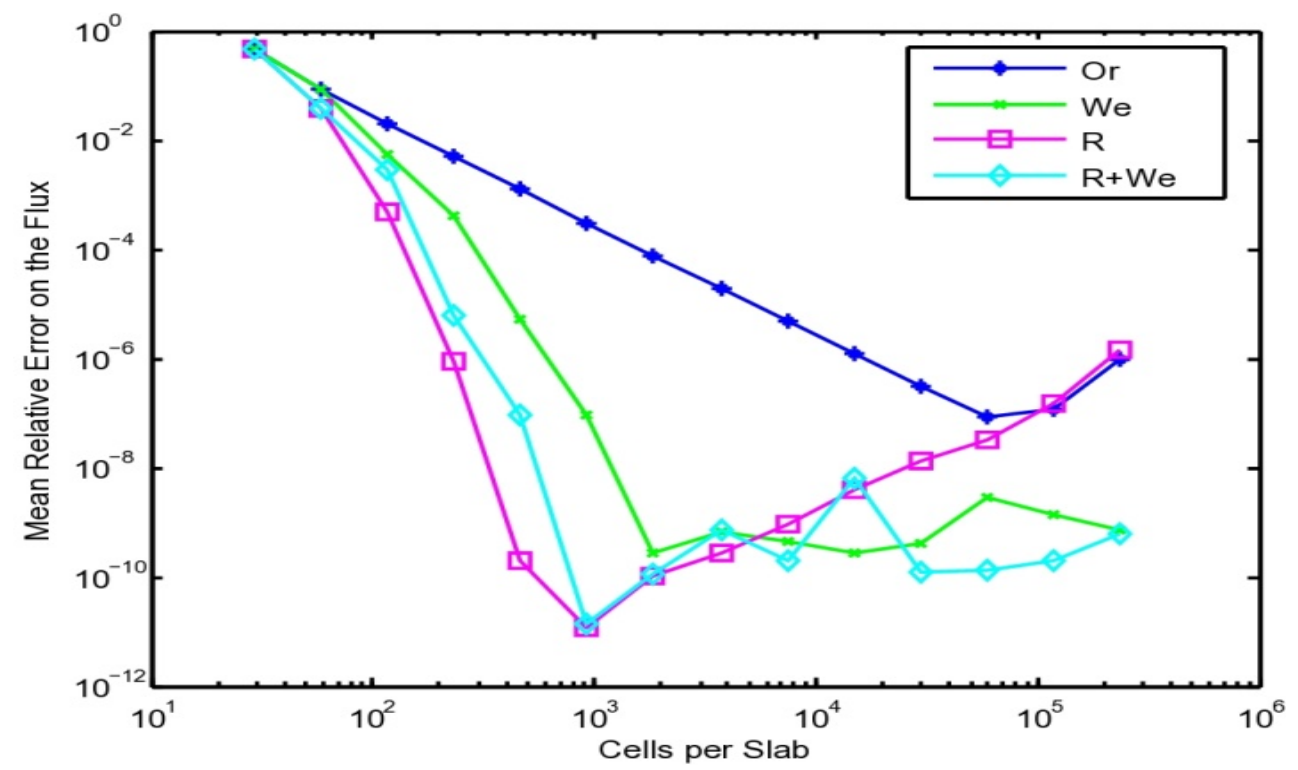

Fig. 2. Behavior of the mean relative error as a function of the number of cells per slab (with increasing iterations) for the 4 solution sequences $(N=10)$. 
solution is not suitable for treating the fine granularity of resonances. Nevertheless, reproducing the spatial variation of the spectrum with such detail and accuracy, even just for a few group calculation, confirms that convergence acceleration is extremely promising not only for diffusion but, as we shall see, also for transport applications as well. Thus, this has to be seen as a first step in including space dependence into FG/UfG benchmark development and should be a guide on how to do FG/UFG in transport theory.

Elia most aptly states his conclusion concerning the analytical form as follows:

......., one final comment needs to be made concerning what has been accomplished here. With a fully analytical solution to the 1D diffusion equation in heterogeneous geometries, one could think that there is no longer a need to use finite difference or finite element methods for the numerical solution of the problem. Given the generality of the new solution presented, there is apparently no need to use an approximate method when the analytical form exists in a concise and elegant fashion.....

Unfortunately, during the implementation of the analytical solution, limits to its applicability have been uncovered. In particular, as anticipated ...., the weak point of this method has proved to be the required inversion of [the eigenvector] matrix..., so it is strictly connected to nuclear data (i.e., [multigroup] parameters). Inverting a matrix is always a delicate numerical procedure; this is particularly true in our case, where the form of the matrix to be inverted is in some way defined by nature and almost any possibility to influence it seems unfeasible.

There is a second difficulty associated with the analytical form. When applied in the panel formalism, the limitation on the diagonalization could be overcome by simply making the panels less than 20 groups each since the analytical form works for this case. Then the analytical solution could be used. However, to maintain the analytical form for panels, the particular solution would have to be found analytically and this has yet to be satisfactorily accomplished.

Finally, even with its shortcomings, as mentioned, the analytical solution can treat few groups in highly heterogeneous media with high accuracy. As we shall see in the FORTRAN development below, multigroup/fine-group solutions to several hundred groups are still possible, which is significant even in today's sophisticated computational environment.

The analytical solution is truly a striking construction, which should have natural potential in the FG/UfG formalism. This has not been found however, which was rather disappointing. For this reason, we have moved forward to consider a 
finite difference implementation, but will not give up on the analytical form so easily.

\section{a2. Finite difference (FD) solution}

\section{(A8.EB.thesis)}

A straightforward finite difference scheme is achieved by simple uniform subdivision defining equidistant knots separated by the step-size $h$ as shown in the following figure:

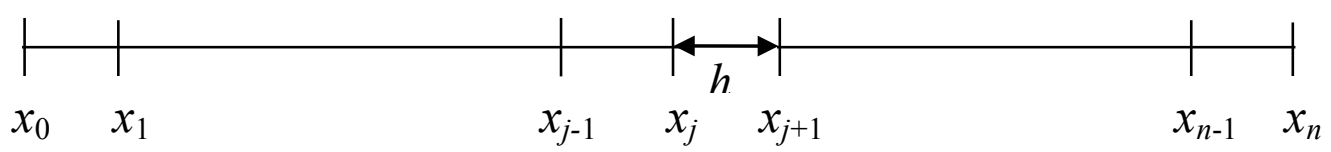

Fig. 3. Uniform grid.

One can now expand each group flux component in a Taylor series on either side of $x$ (at $x \pm h)$ to give

$$
\begin{gathered}
\boldsymbol{\phi}(x \pm h)=\boldsymbol{\phi}(x) \pm h \boldsymbol{\phi}^{(1)}(x)+\frac{h^{2}}{2} \boldsymbol{\phi}^{(2)}(x) \pm \frac{h}{3 !}^{3} \boldsymbol{\phi}^{(3)}(x)+ \\
+\mathbf{O}\left(h^{4}\right) .
\end{gathered}
$$

By adding these equations and truncating the series at the fourth term, we deduce the following cell centered finite difference approximation:

$$
\phi_{\mathbf{j}+1}-\boldsymbol{\alpha} \phi_{\mathbf{j}}+\phi_{\mathbf{j}-\mathbf{1}}=-h^{2} \mathbf{q}_{\mathbf{j}}
$$

The source has been redefined to include the inverse diffusion matrix and $\alpha$ is the $G \times G$ block

$$
\boldsymbol{\alpha} \equiv 2 \mathbf{I}-h^{2} \mathbf{B}^{2}
$$

The unknowns are the $G$ - energy components of the flux for each knot of the spatial mesh, assumed to number $n+1$. While the monoenergetic problem leads to a three-diagonal matrix connecting regions, in the multigroup problem we obtain a block triangular vector equation, whose diagonal elements are $G \times G$ matrices. The solution then becomes a tri- diagonal block matrix inversion. The above three-term recurrence persists within each slab of a heterogeneous medium. Interestingly, both the analytical and FD forms lead to a three-term recurrence at internal interfaces, but with different coefficients.

The one distinct advantage of the FD from of solution is that convergence acceleration can be readily used to achieve high accuracy. This is done by 
solving the FD form with reduced discretization by a factor of two and using either the Richardson or Wynn-epsilon [3] accelerators to accelerate convergence. Richardsons acceleration simply sequentially eliminates higher order error terms to obtain higher accuracy discretizations for the group flux at each spatial point and gives the more accurate solution

$$
R_{k, l}=R_{k, l-1}+\frac{R_{k, l-1}-R_{k-1, l-1}}{2^{2 l}-1},
$$

where $R_{k, l}$ is the $g$-group flux at position $x_{j}$ after the discretization has been reduced by a factor of $2^{k}$. $R_{0,0}$ is the original finite difference solution to $\mathrm{Eq}(1)$. The true solution is then obtained as the discretization approaches zero. This is the same as the limit $\lim _{k \rightarrow \infty} R_{k, l}$ and will generally converge more rapidly than the original FD sequence (for $h / 2^{k}$ ); hence, Richardsons sequence accelerates convergence. The second sequence accelerator, Wynn-epsilon acceleration, is given in the following form:

$$
\begin{aligned}
& \epsilon_{-1}^{(n)}=0, \\
& \epsilon_{0}^{(n)}=s_{n}, \quad n=0, \ldots, N \\
& \epsilon_{k}^{(n)}=\epsilon_{k-2}^{(n+1)}+\frac{1}{\epsilon_{k-1}^{(n+1)}-\epsilon_{k-1}^{(n)}}, \quad k=1, \ldots, N ; \quad n=0, \ldots, N-k,
\end{aligned}
$$

which leads to the tableau

$$
\begin{aligned}
& \epsilon_{0}^{(0)} \quad \epsilon_{1}^{(0)} \quad \epsilon_{2}^{(0)} \quad \ldots \quad \epsilon_{N-1}^{(0)} \epsilon_{N}^{(0)} \\
& \epsilon_{0}^{(1)} \quad \epsilon_{1}^{(1)} \quad \epsilon_{2}^{(1)} \quad \ldots \epsilon_{N-1}^{(1)} \\
& \begin{array}{cccc}
\epsilon_{0}^{(2)} & \ldots & \ldots & \cdots \\
\ldots & \ldots & \epsilon_{2}^{(N-2)} &
\end{array} \\
& \ldots \epsilon_{1}^{(N-1)} \\
& \epsilon_{0}^{(N)}
\end{aligned}
$$

$s_{n}$ is the original sequence and $\varepsilon_{k}^{(n)}$ is an estimate of the limit. 
As an initial test case, again, a symmetric three-region reactor is assumed but with a central depleted U/Carbide region and graphite reflectors. A uniform source emits in the top group in the central region. There are 15 initial cells per slab and the energy range is $[3 \mathrm{eV}, 800 \mathrm{eV}]$. The slab configuration properties are given in Tables $2 \mathrm{a}, \mathrm{b}$. We now consider a fine-group calculation of 5000 groups.

As the mesh size is reduced, we find Fig. 4, which shows the spatially averaged error over all groups between iterates versus number of cells in the $\mathrm{U} / \mathrm{C}$ and graphite slabs. As can be observed the Richardson extrapolation gives an

Table 2a. slab geometrical and physical properties.

\begin{tabular}{cccccc}
\hline$m$ & Name & Compound & $\begin{array}{c}\text { Thickness } \\
{[\mathrm{cm}]}\end{array}$ & & $\begin{array}{c}\rho \\
{\left[\mathrm{g} / \mathrm{cm}^{3}\right]}\end{array}$ \\
\cline { 1 - 3 } \cline { 6 - 6 } & $\mathrm{F}$ & $\mathrm{UC}$ & 5 & & 13.60 \\
2 & $\mathrm{Gr}$ & $\mathrm{C}$ & 15 & & 2.26 \\
\hline
\end{tabular}

Table 2b. slab geometrical and physical properties.

\begin{tabular}{cccccc}
\hline$m$ & Name & Compound & $\begin{array}{c}\text { Thickness } \\
{[\mathrm{cm}]}\end{array}$ & $R$ & $\begin{array}{c}\rho \\
{\left[\mathrm{g} / \mathrm{cm}^{3}\right]}\end{array}$ \\
\hline 1 & $\mathrm{~F}$ & $\mathrm{UC}$ & 5 & 15 & 13.60 \\
2 & $\mathrm{Gr}$ & $\mathrm{C}$ & 15 & 15 & 2.26 \\
\hline
\end{tabular}




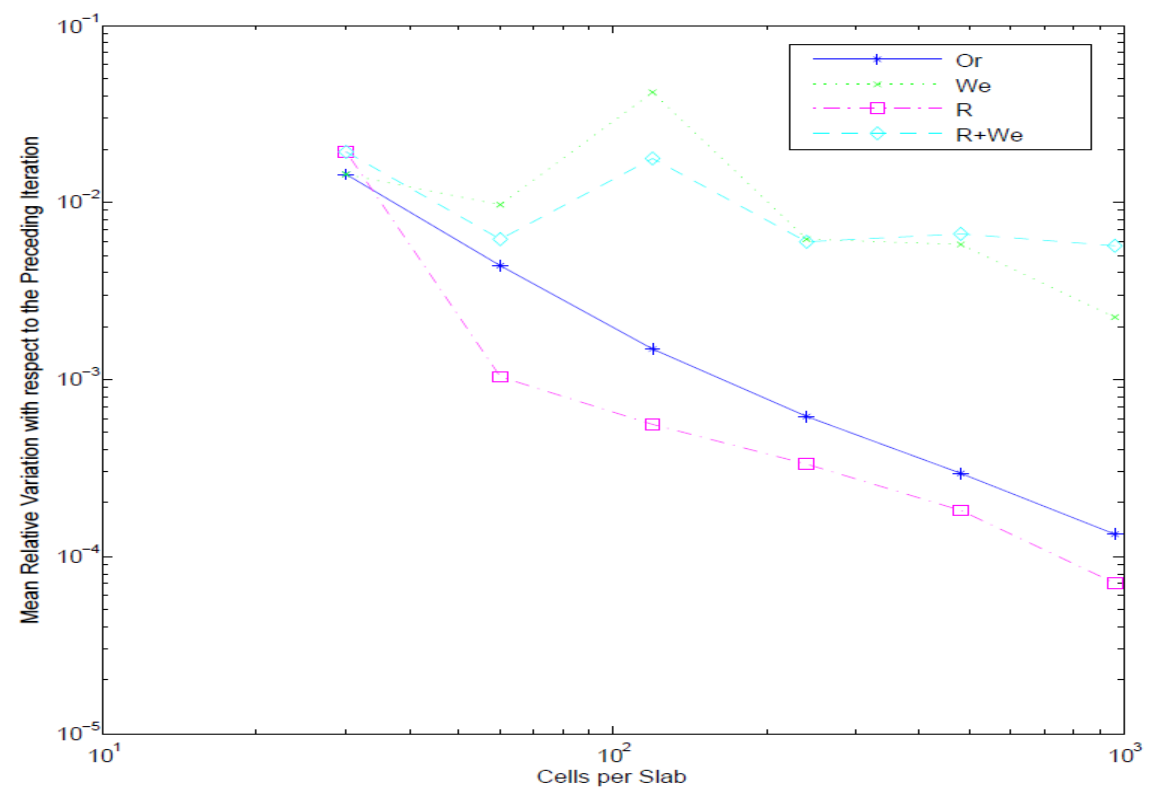

Fig. 4. Application of sequence accelerators.

improvement of about a factor of about two, which is not particularly encouraging. Also, it is not clear why $W$-e behaves so poorly.

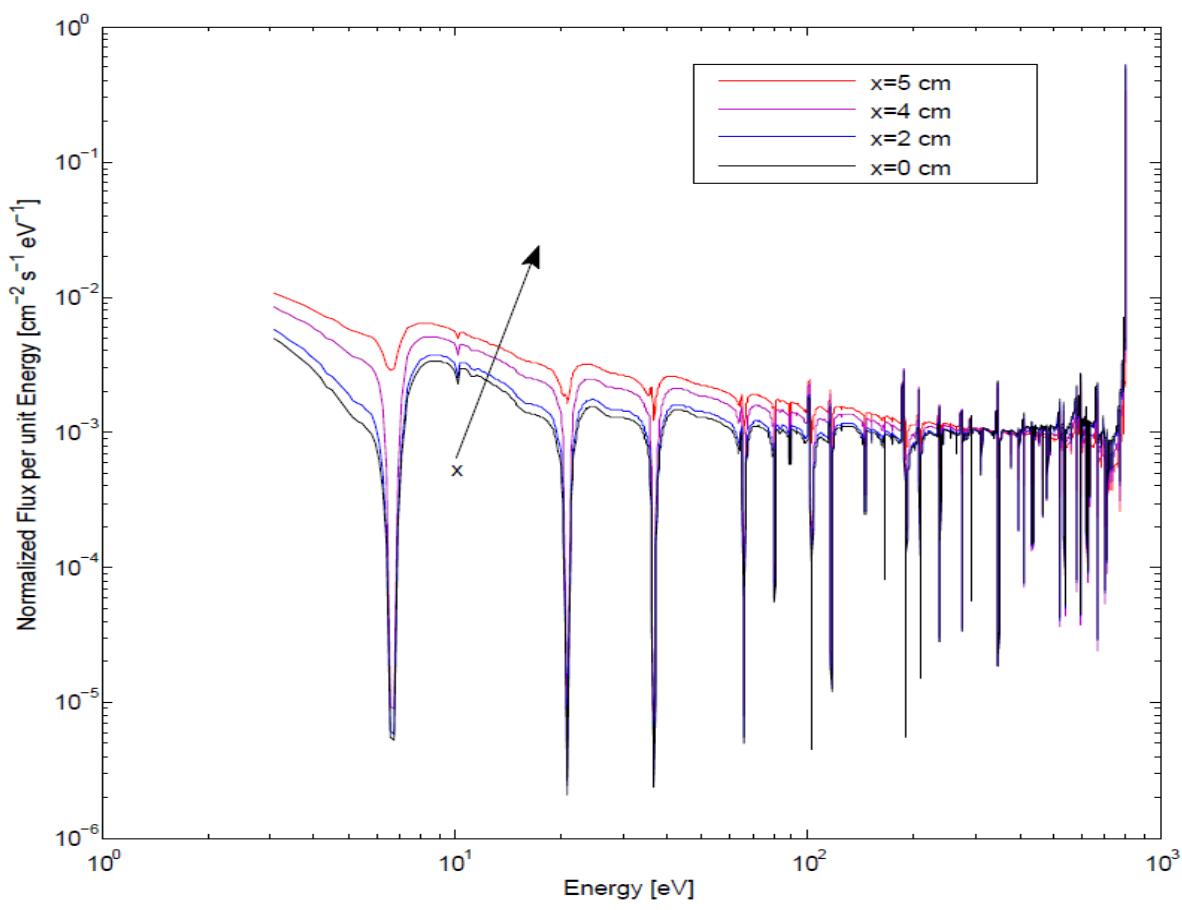

Fig. 5a. Spectrum across the fuel. 


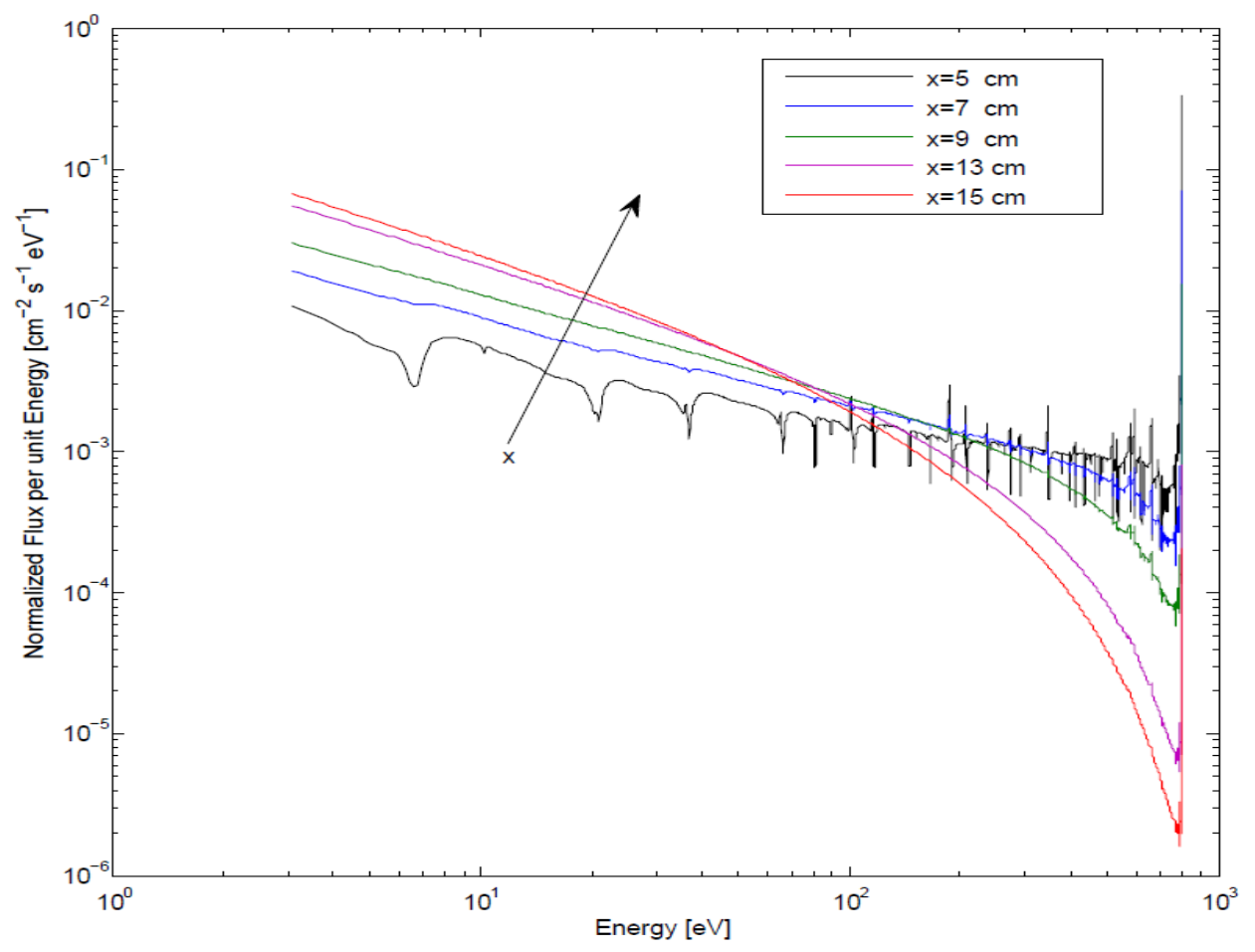

Fig.5b. Spectrum across the reflectors.

In any case, we plot the spectral behavior in Figs. 5a,b across the slabs. These figures are included to observe the change in the spectrum as one moves from a material with many resonances to one with none. In particular, the spectrum for the reflector shows the same resonant behavior as the fuel near the interface. This is what makes homogenization so ineffective since one is averaging over large spectral variation unless the spatial grid is fine enough to capture the variation.

From this investigation, one concludes that convergence acceleration has the potential to provide a distinct advantage over the central FD scheme, which indeed does admit FG solutions; while, MATLAB's implementation of the analytical solution does not.

We now consider the FORTRAN implementation of the analytical and FD solutions.

\section{b. Analytical/Numerical diffusion theory: FORTRAN implementation b1. Analytical solution}

Most likely, the relatively poor performance of the analytical solution is mostly a result of using MATLAB. Even though MATLAB is optimized for matrix operations, it is quite slow for this application and therefore one has to sacrifice 
either in number of groups or in spatial resolution for reasonable computational efficiency. It is prudent therefore to code the analytical solution in FORTRAN, which is the goal of this section. We presented part of this material at the 2009 Hollywood Florida ANS Meeting A.9 Summary and A.10 presentation.

\section{b1.1. Numerical implementation}

There are two primary numerical procedures to be implemented for the evaluation of the analytical form of Eq(a1.1.6a). The first is the diagonalization of the interaction matrix $\mathbf{B}_{j}^{2}$. While seemingly a straightforward matter, this turns out not to be the case as discussed in the last section. The fundamental requirement for diagonalization is that the eigenvectors of the matrix be distinct, which is true when the eigenvalues are distinct. Certainly, for the multigroup diffusion application, this will be the case and therefore all that is required is to solve for the eigenvalues of the interaction matrix. We have identified several eigenvalue solvers including a sequence from Numerical Recipes [4] and the LAPACK option of the LAHEY compiler [5]. The LAPACK option has the advantage that it returns the eigenvectors and is therefore the preferred method. However, for pure down scatter, the eigenvalues are simply the diagonal elements and the eigenvectors follow directly from matrix inversion.

The eigenvalues and eigenvectors are then part of the determination of the expression

$$
\boldsymbol{H}_{j}^{ \pm}(x) \equiv \boldsymbol{\alpha}_{j} \boldsymbol{h}_{j}^{ \pm}(x) \boldsymbol{\alpha}_{j}^{-1}
$$

central to the evaluation of the analytical solution. This seems to require the determination of the inverse of $\boldsymbol{\alpha}_{j}$. However, knowing $\boldsymbol{\alpha}_{j}$ and $\boldsymbol{h}_{j}^{ \pm}(x)$ allows

$$
\boldsymbol{\alpha}_{j}^{T} \boldsymbol{H}_{j}^{ \pm}(x)^{T} \equiv\left[\boldsymbol{\alpha}_{j} \boldsymbol{h}_{j}^{ \pm}(x)\right]^{T}
$$

to be solved for $\boldsymbol{H}_{j}^{ \pm}(x)^{T}$ by LU decomposition, giving $\boldsymbol{H}_{j}^{ \pm}(x)$ on transpose. Thus, one avoids an explicit determination of the inverse of $\boldsymbol{\alpha}_{j}$.

The second numerical procedure is the solution of the block triangular recurrence relation at slab interfaces

$$
\boldsymbol{M}_{j} \boldsymbol{\phi}_{j}-\boldsymbol{N}_{j} \boldsymbol{\phi}_{j-1}-\boldsymbol{P}_{j} \boldsymbol{\phi}_{j-2}=\boldsymbol{f}_{j}, 2 \leq j \leq n,
$$


where the coefficient matrices $\boldsymbol{M}_{j}, \boldsymbol{N}_{j}, \boldsymbol{P}_{j}$, and $\boldsymbol{f}_{j}$ are known [6]. The solution is found most efficiently using a block triangular solver with iterative error estimation.

\section{b1.2. Numerical demonstration}

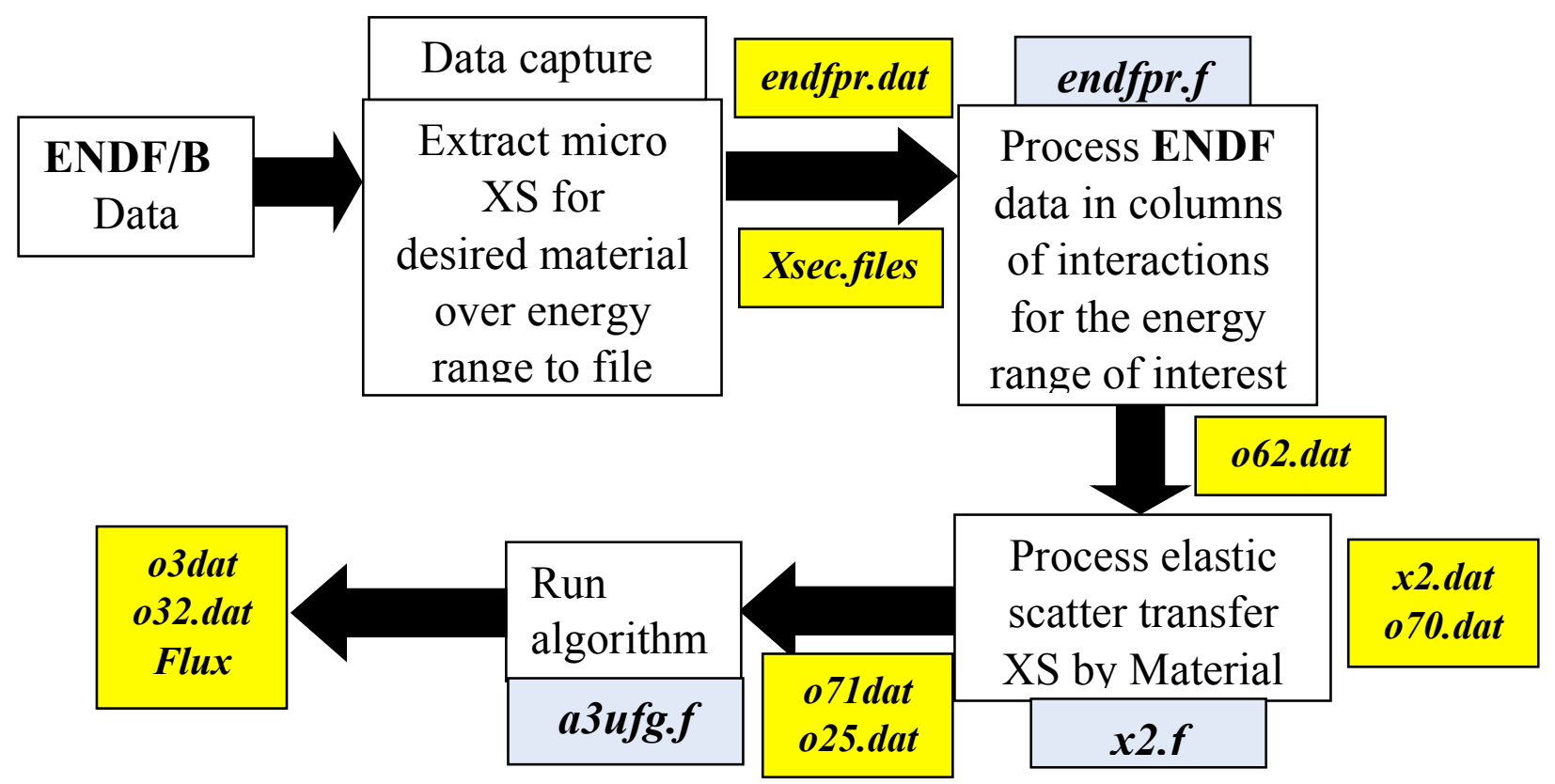

Fig. 1. Flow Chart for the analytical diffusion.

For all demonstrations to follow, homogenous mixtures of fuel and water slabs with a fixed source are assumed. The intent of the demonstration is to show clearly the weakness and strength of the analytical solution.

As shown in Fig.1, the analysis proceeds in several steps, the first of which is the generation of a 64-group cross section set from the ENDF/B cross section library as described in Task 1. The energy range to be investigated is from $0.1 \mathrm{eV}$ to $10 \mathrm{eV}$. It should be emphasized that in this region, absorption in water is relatively small giving rise to an instability in the diagonalization of the interaction matrix for a pure water slab as will be shown.

The second step is the generation of the scattering block assuming elastic scattering, also described in Task 1. A summation of the out-scatter cross sections to all groups has been included as a check on the scattering block. The sum should be the total scattering cross section by group, which is true for nearly all but the last few groups for all elements except elemental hydrogen. The discrepancy arises from the $1 / E$ weighting spectrum, the granularity of the group structure and lack of a catchall dump group. 
The first case considered is for a slab with zero current at the centerline and zero flux at the outer boundary. The slab thickness is $0.5 \mathrm{~cm}$ with a uniform source in the first group over half the slab thickness from the centerline. For simplicity, fission returns neutrons uniformly to all groups, but any fission distribution can be considered. By other means, the critical dimension is found to be $0.52750 \mathrm{~cm}$ so the slab is surely subcritical.

Figure 2 shows the flux spectrum across the slab for decreasing fuel volume fraction (VfF) and correspondingly increasing water volume fraction. Notably, the flux takes on the spectral characteristic of the water, as one would expect with reduced fuel volume fraction.

If, however, we consider a slab of pure water, the analytical solution gives an entirely unrealistic flux distribution as shown in Fig. 3. This is a predictable numerical phenomenon. Table 1 gives the condition numbers for the matrix diagonalization for the fuel volume fractions considered. As observed, the more pure water-like the slab is, the greater the condition number and the more illconditioned the matrix diagonalization is. This is a result of essentially zero absorption of the water causing the elements of the interaction matrix to differ by factors of more than $10^{40}$. With double precision arithmetic, inversion of such a matrix is not possible. However, when the absorption associated with just $10^{-6}$ volume fraction of fuel is added, the calculation stabilizes as shown in the graphical inset in Fig. 3.

The final demonstration is for a heterogeneous slab composed of the three slabs of thicknesses $0.2 \mathrm{~cm}, 0.35 \mathrm{~cm}$ and $0.2 \mathrm{~cm}$ with fuel fractions 0.850 .05 and 0.85 respectively. The configuration is repeated 48 times to give a 144 region heterogeneous medium. There is a uniform source emitting in the top group in every other slab, except for two slabs where the source is in group 23. Figure 4 shows the flux for every $4^{\text {th }}$ group of a $200 \mathrm{~s}$ calculation on a quad-core $2.5 \mathrm{GHz}$ $\mathrm{VAIO} /$ Sony PC. The evaluation is stable for this large diffusion problem and the positions of the asymmetric sources are clearly visible. 

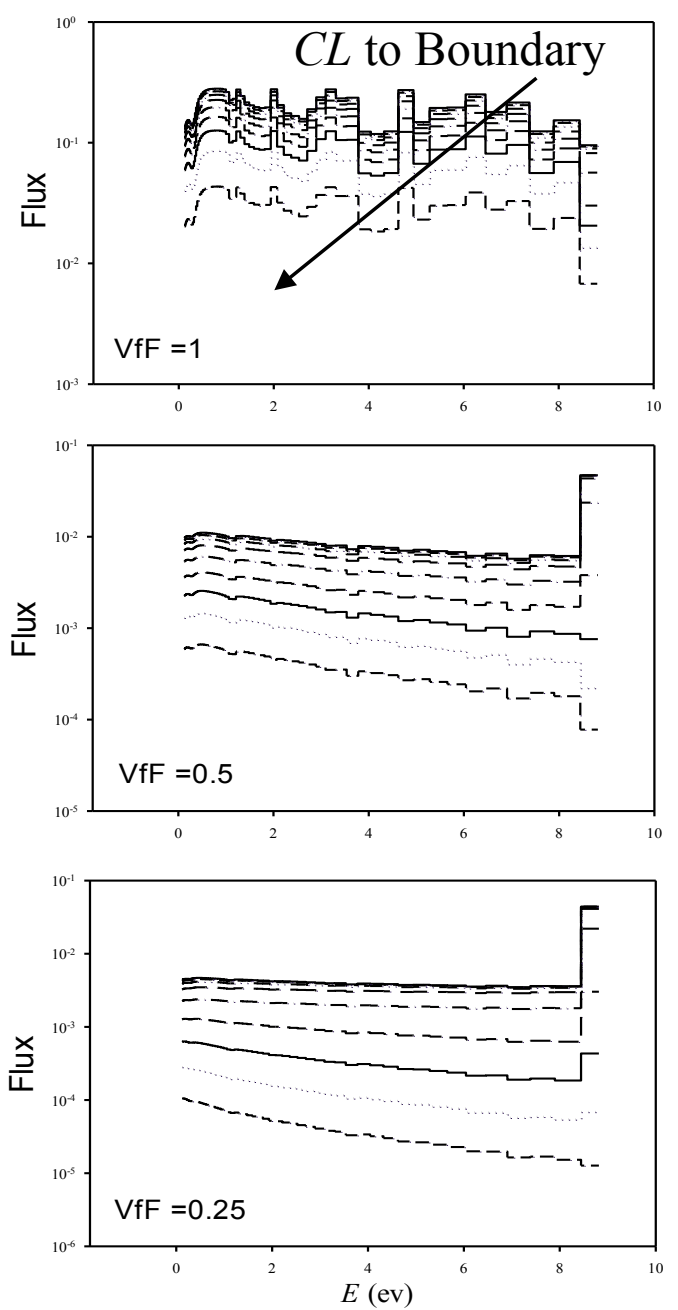

Fig. 2. Flux in a single U235/water slab with increasing volume fraction of water.

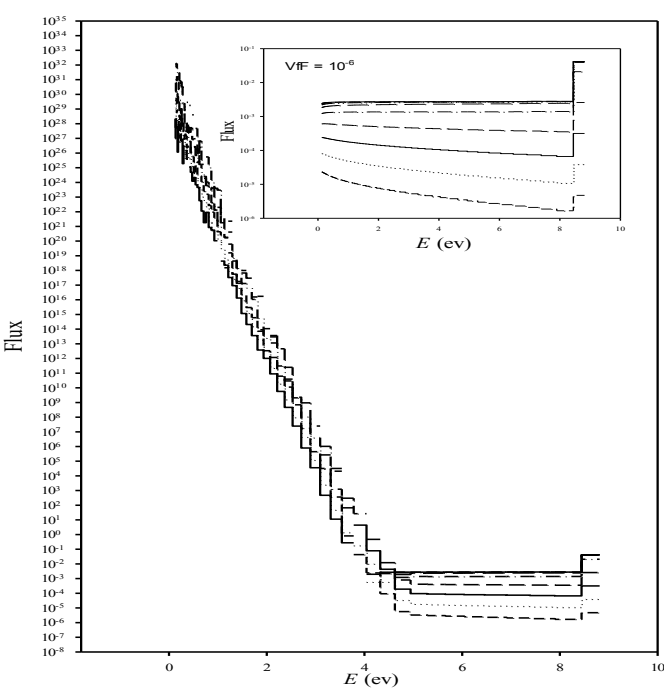

61 
Fig. 3. Failure of the analytical solution for a single water slab.

Table 1

Condition numbers for matrix diagonalization

\begin{tabular}{cc}
\hline VfF & $\begin{array}{c}\text { Condition } \\
\text { Number }\end{array}$ \\
\hline 1.0 & 81.9 \\
0.5 & $1.03 \times 10^{5}$ \\
0.1 & $1.09 \times 10^{6}$ \\
0.01 & $5.77 \times 10^{7}$ \\
$10^{-6}$ & $9.17 \times 10^{11}$ \\
0.0 & $8.19 \times 10^{50}$ \\
\hline
\end{tabular}

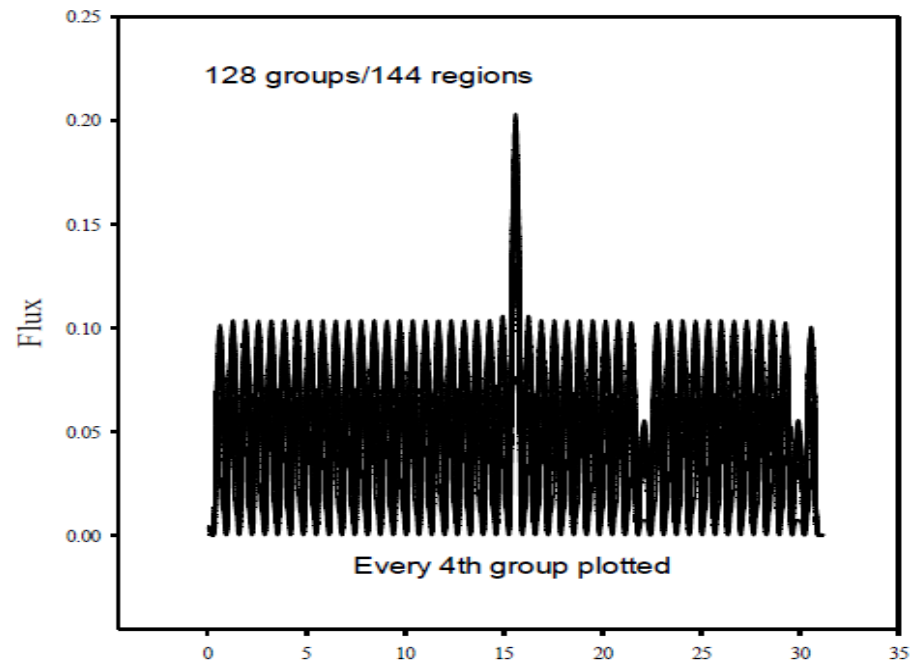

Fig. 4. An analytical diffusion demonstration.

\section{b1.3. Remarks}

While the use of the analytical evaluation will most likely not be possible for a full 70K UFG application, its viability as a reliable analytical solution has been demonstrated here for multigroup and fine-group. Arguably, the calculation for Fig. 4 is most likely the largest of its kind to date. The analytical solution presented, should eventually find its way into the classroom as it represents a true analytical solution to a relatively realistic problem.

\section{b2. Finite difference solution}

\section{(A11.FD.Diff.paper and A12.FD.Diff.Pres) (A13.NT.Thesis)}


With a demonstrated analytical multigroup diffusion calculation for 64-groups, we are in a position to construct a FD solution and test acceleration using the analytical solution for verification as was done with the MATLAB version.

In this section, we conduct several numerical experiments concerning convergence acceleration of the FD approximations implementing Eq(a1.1.6a). Before giving the results, we provide some practical details concerning how one performs the accelerations and comparisons.

Vector sequence acceleration is performed by applying the scalar extrapolation techniques described above to each flux component. Each vector component is considered a single isolated term as we will be accelerating many sequences simultaneously. The un-accelerated components will therefore be those referred to as the original edits of the starting grid (initial discretization level). To compare accelerated FD results and the analytical solution, we have implemented an average relative error over components, which is simply the average of the relative errors of each flux component divided by the number of components.

Performing convergence acceleration of a sequential discretization involves two sources of error. The first is the truncation error that comes directly from keeping only a finite number of terms in the Taylor expansion responsible for the FD algorithm. If stable, the error tends theoretically to zero for an infinitesimal step size. The second is rounding error due to finite machine precision. This greatly influences our accelerations after a certain point, leading to erratic behavior because of subtraction of similar numbers. In the Richardsons extrapolation, we will observe an error explosion after an absolute minimum in the relative error. In Wynn-epsilon $(W-e)$ extrapolation, unstable oscillations also similarly occur. Both instabilities result from numerical round off. In particular, in $W$-e, division by zero can occur and therefore the algorithm must be stopped before this occurs.

Numerical calculations have been performed using FORTRAN programming. Since our primary goal is to develop benchmark solutions using conventional machines, we performed our simulations using a laptop equipped with an Intel Core i7-2630QM processor and 6GB RAM. We used two algorithms written in double precision. The first one evaluates analytical solutions to the 1D multigroup diffusion equation in a heterogeneous medium as in §II.3.1.b.1; the second performs the FD calculations. For the matrix formulation, the block triangular solver (matrix "two sweeps" algorithm) has been implemented using LAPACK [5]. 
Group parameters were determined from the ENDF database as described in Task 1.

\section{b2.1. Multigroup calculations and comparison to analytical solutions}

We start by simulating a single-slab reactor, made of a homogeneous mixture of $10 \%$ U235 and 90\% light water and imposing zero current boundary conditions. We apply a superimposed unit source in the first energy group throughout the slab (energy range $0.1 \mathrm{eV}-10 \mathrm{eV}$ ). In Fig. 1, the true relative error compared to the analytical solution is reported for a 5-group calculation, where for demonstration

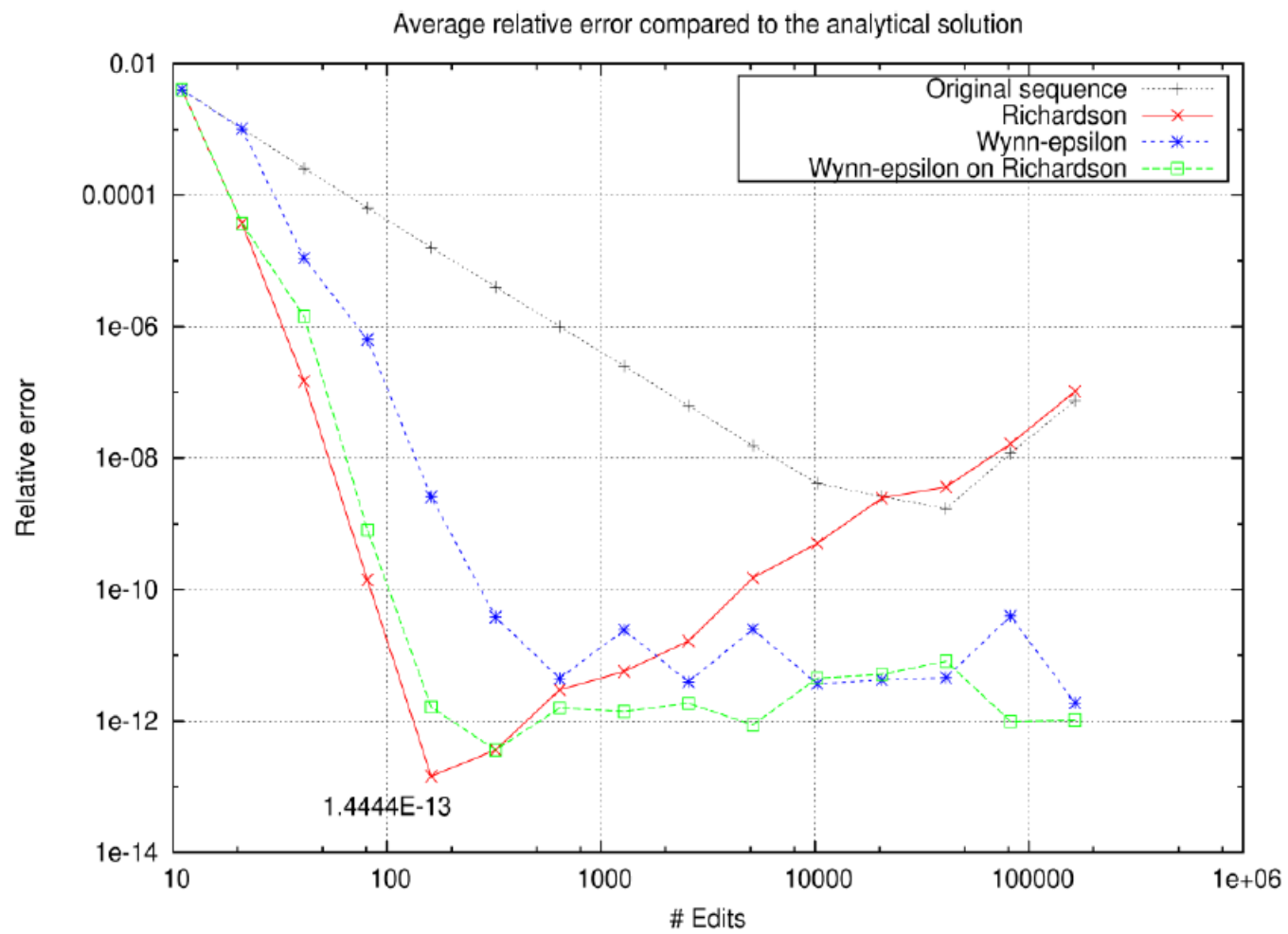

Fig. 1. Spatial average relative error compared to the analytical solution for a 5-group-single slab simulation.

purposes, we forced 15 grid iterations starting from an original mesh of 11 points.

For this simulation, Richardsons extrapolation gives the best relative error $\left(1.4 \times 10^{-13}\right)$ in just 4 grid refinements similar to what was found in $\S I I . a 1.1$. The magnitude of the relative error is indicative of a numerical quality representative of semi-analytical benchmarks. We repeated the calculation to verify if the acceleration procedure is self-consistent, satisfying a practical convergence 
criterion based on the average relative error when referred to the previous iteration since, in general, the analytical solution will not be known. We obtained self-convergence with the same trends and errors of the same magnitude as shown in Fig. 1.

Since numerical evaluation of the analytical solution for many groups (more than 64) are not readily available unless only absorbing materials are considered, we performed a three-slab 64-group simulation using 30\%-enriched $\mathrm{UO}_{2}$ as fuel and $10 \%$-borated water as moderator. In Fig. 2, the relative error of the accelerated MG/FD scheme is presented as compared to the analytical solution. In all cases, we see the accelerated sequences approaching the analytical solution significantly more rapidly than the original sequence, constituted by the original solution obtained through successively finer grid subdivision.

During our simulation campaign, we encountered some difficulties comparing the two solutions when small fluxes values (of the order of $10^{-5} \mathrm{n} / \mathrm{cm}^{2}-\mathrm{s}$ ) were involved, in particular, for fine meshes and for highly absorbing media. Another issue was the tendency of the numerical evaluation of the analytical solution to be affected by ill-conditioning for purely scattering media as referred to in the previous section. No ill-conditioning verification has been carried out for the FD schemes at this point other than in $\S b 1$ above. In any case, we conclude as we did earlier that convergence acceleration is quite effective and that the error between iterations is representative of the true error.

\section{b2.2. Ultra-fine-group calculation for pure slowing down}

In addition, we performed a 10K-group calculation for a three-slab system made of a central fuel slab of 10\%-enriched Uranium Dioxide, and two external water slabs, as shown in Fig. 3. We considered the energy range of $0.1 \mathrm{eV}-10 \mathrm{eV}$. In Figs. 4 and 5, the flux per unit energy in water and in fuel and the relative error as compared to the previous iteration are reported. Convergence acceleration (here, only for Richardsons extrapolation) permits self-convergence significantly more rapidly than the original sequence. Indeed, convergence acceleration enables accuracy to better than six places in less than 1000 points. This is one of the first demonstrations of a FG (Fine-Group) converged accelerated semianalytical diffusion benchmark solution. 


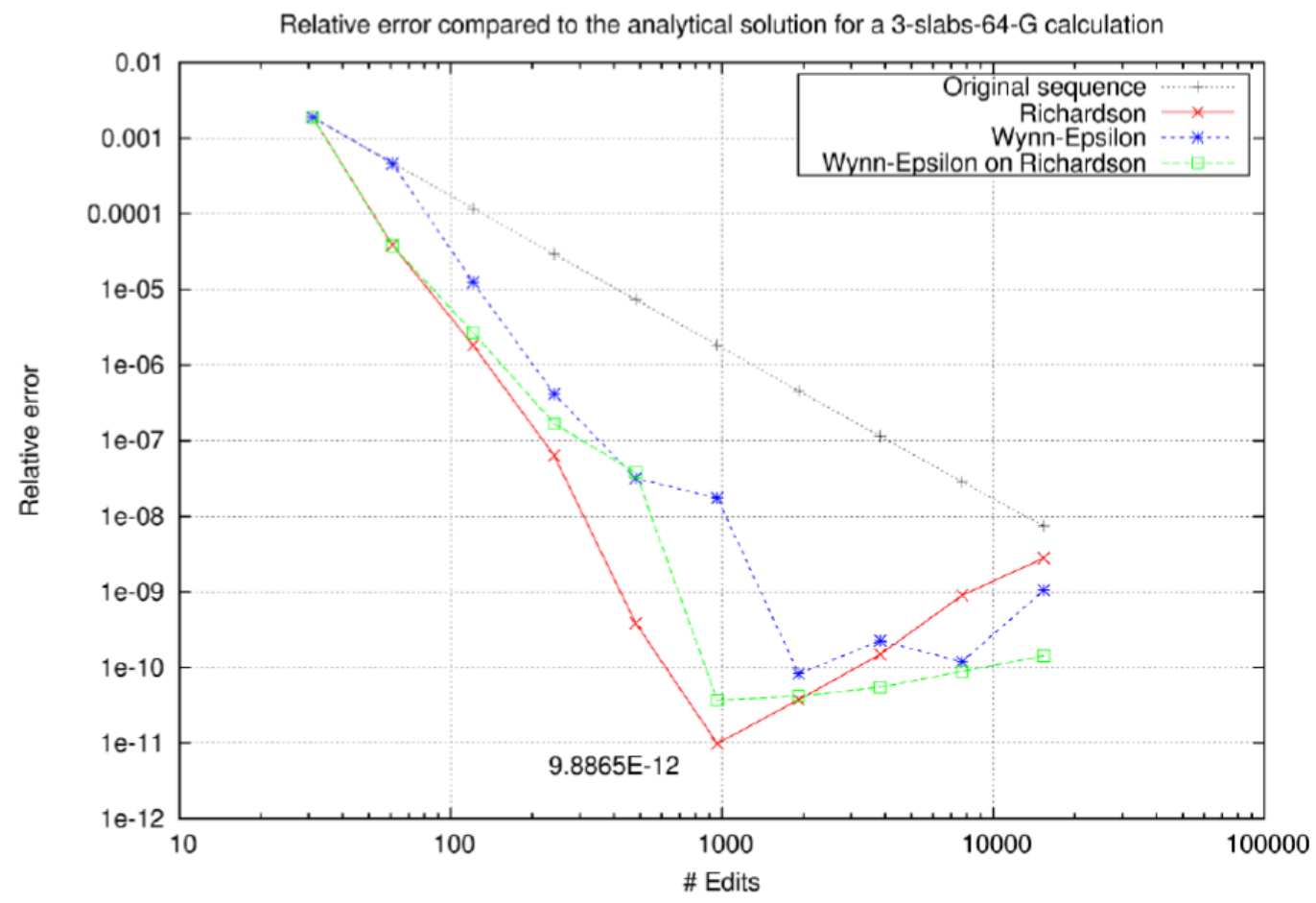

Fig. 2. Comparison for a three-slabs system and 64 energy groups.

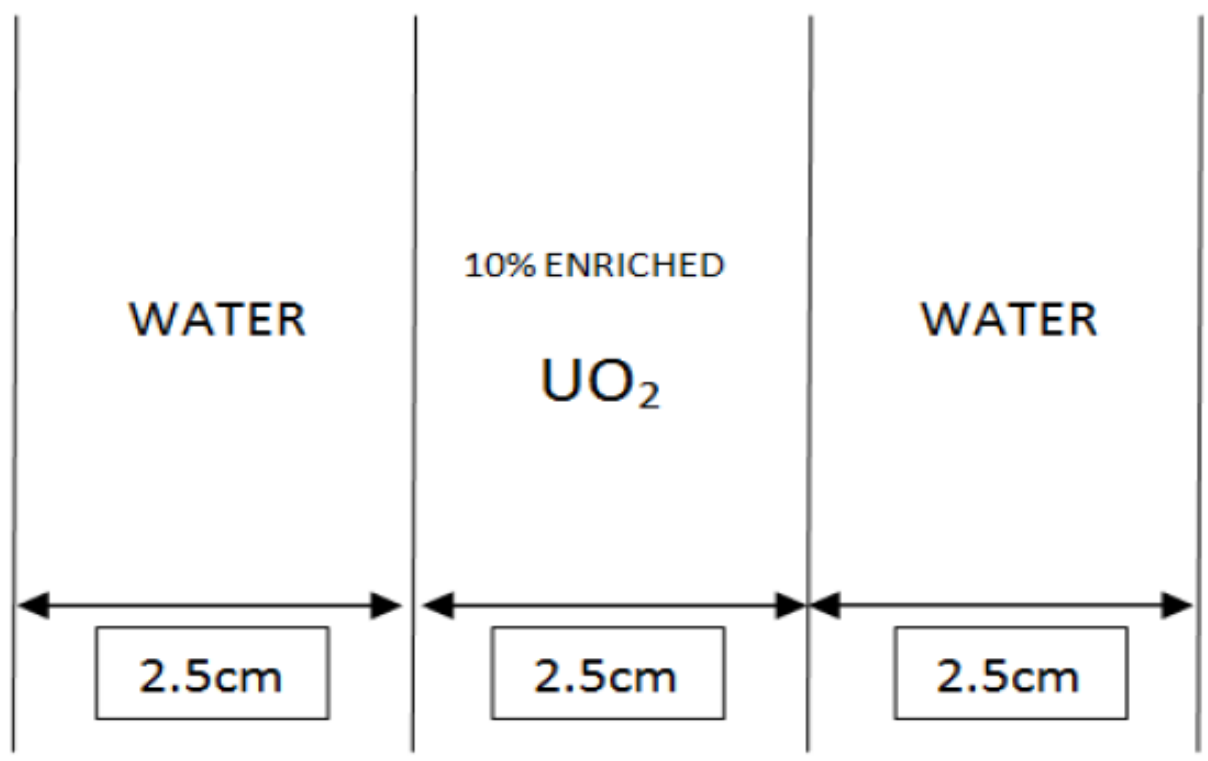

Fig. 3. System used in the ultra-fine simulations.

Next, we attempt a 20K UfG calculation for the three-slab system. In Fig. 6, the flux per unit energy in fuel and in water is presented. Using convergence acceleration, we obtain a self-consistent relative error of $3.4 \times 10^{-6}$ at the fifth iteration using Richardsons extrapolation with 481-edits; while, the original 
sequence produced a $1.6 \times 10^{-3}$ at the same iteration. The calculation took $23,811 s$, acceptable for a one-time semi-analytical benchmark calculation. It must be

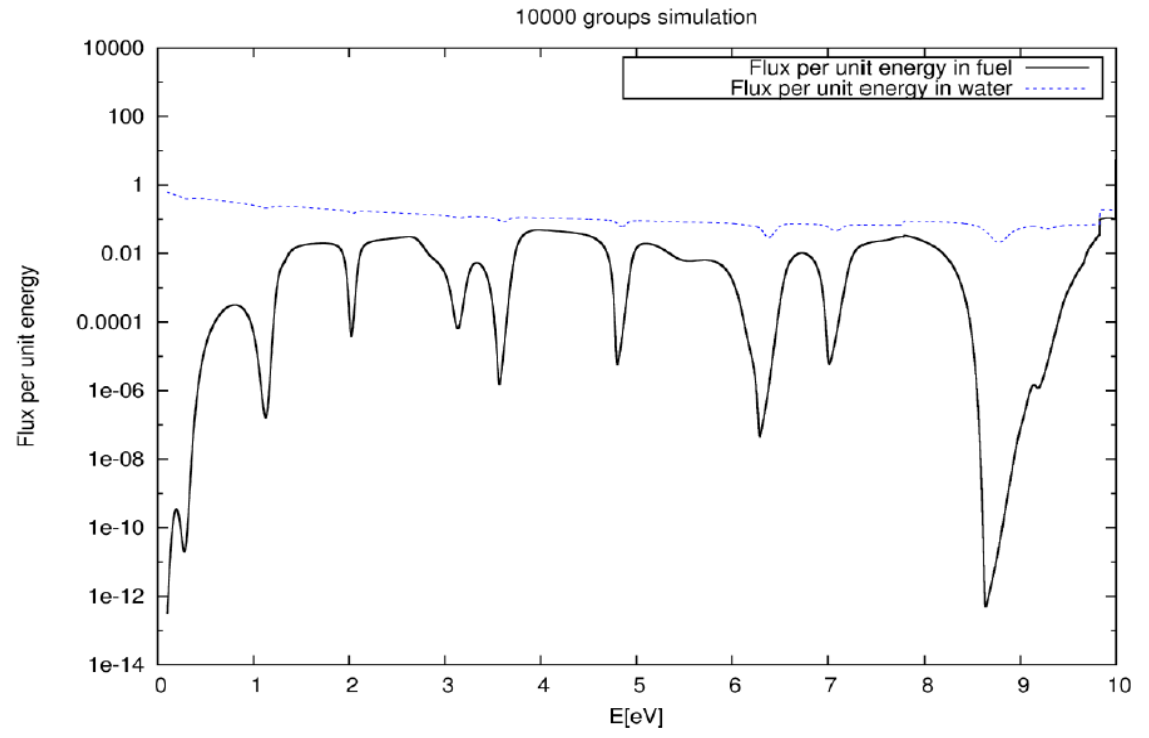

Fig. 4. 10K-group simulation for a three-slabs heterogeneous system. Flux per unit energy in fuel and water.

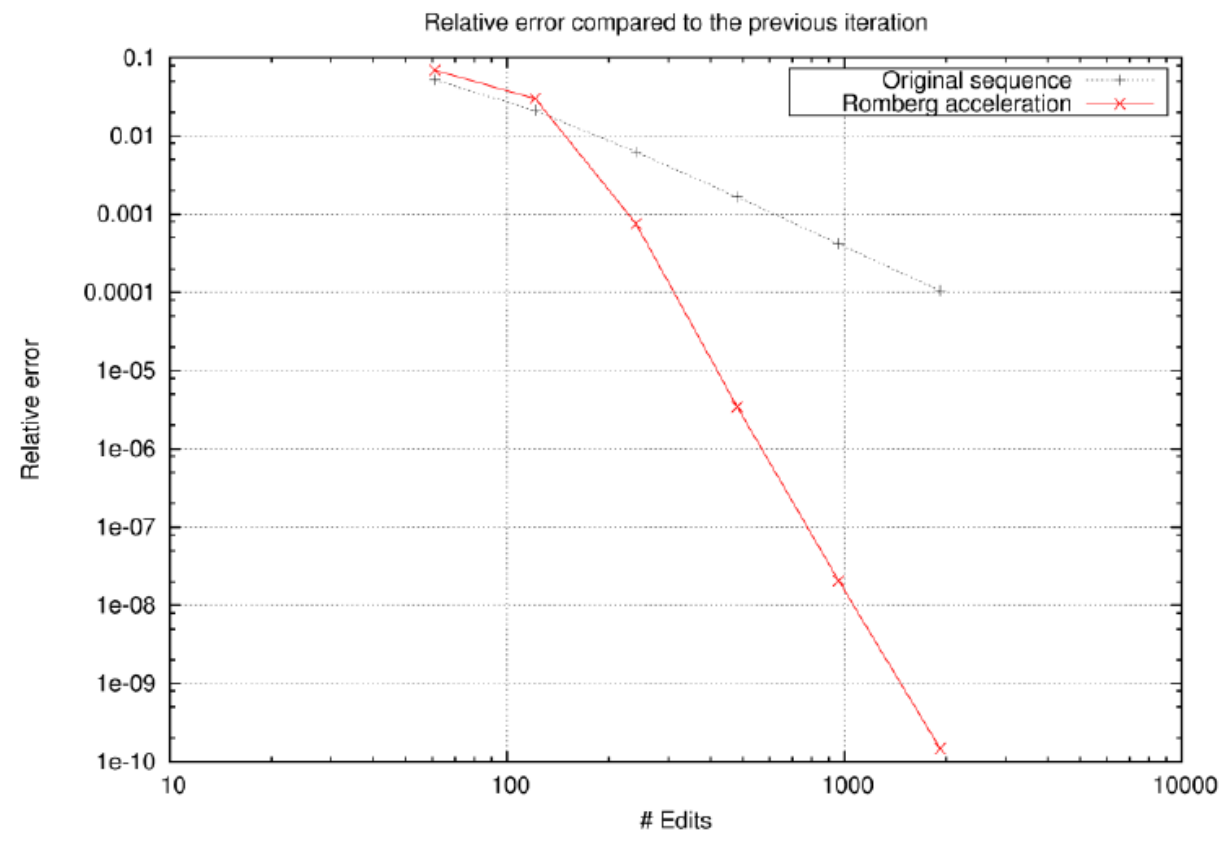

Fig. 5. Relative error compared to the previous iteration for the 10K-group simulation. 


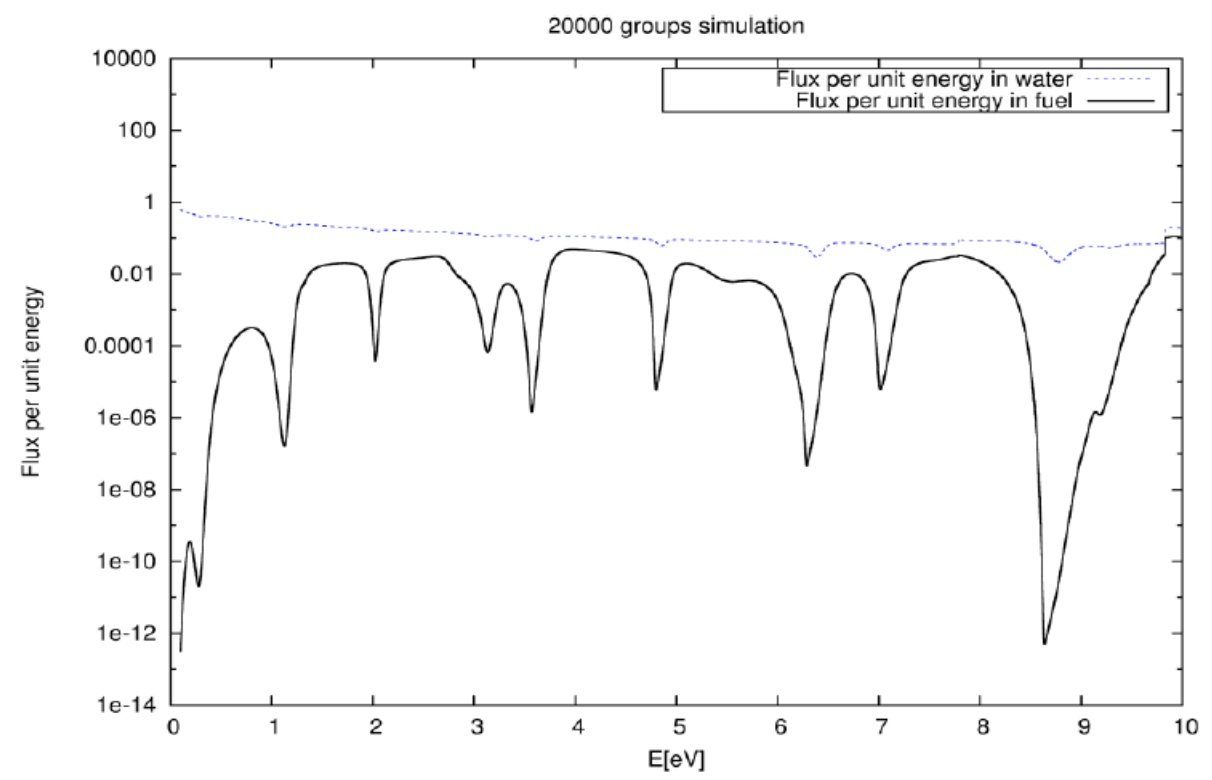

Figure 6. 20K-UfG simulation for a three-slabs heterogeneous system. Flux per unit energy in fuel and water.

remarked that this example did not use the panel formalism per se, as it used a single group-by-group cascade. In any case, this is an example of a diffusion UfG at a practical error and meets one of our task objectives, but still requires excessive CPU time and is further indication of the need for HPC.

\section{b2.3. Remarks}

We have seen how convergence acceleration enables numerically generated semi-analytical multigroup/Fine-group diffusion benchmarks. This was empirically shown through simple examples by comparison to the few- and finegroup analytical solutions. In double precision, we obtained average relative errors for the flux components on the order of $10^{-12}$ for multigroup calculations (64-group ) and multiple slabs of different materials. Since accurate evaluations of the analytical solution are not available for more than several hundred groups at this time, further demonstrations are needed for fine- and ultra-fine energy meshes. Nevertheless, self-convergence has always been achieved in our examples. Convergence acceleration, in fact, allows us to achieve practical selfconvergence in a few grid discretizations even for ultra-fine energy meshes using a cascade procedure (group by group) in a purely slowing down problem. However, it is obvious that HPC will be required for UFG calculations to become routine.

\section{c. Analytical diffusion theory: GPU implementation}

With the development of more affordable, multicore GPUs, highly parallel mathematical problems can be solved in a fraction of the time. Large matrix 
operations is one area that is well suited for GPU implementation. With the acquisition of an NVIDIA Tesla C2075, with 448 CUDA cores, the system can realize 515 Gflops of peak double precision floating-point performance. In this way, we hope to bring supercomputing speed to a standard PC. We had envisioned solving the multi-group problem to upwards of tens of thousands of groups with MATLAB's optimized matrix operations and tight GPU integration however, this did not occur. A basic analytical diffusion solution was programmed on the GPU. The multigroup analytical solution

$$
\boldsymbol{\phi}(x)=\left[\boldsymbol{I}-\boldsymbol{\alpha} \boldsymbol{h}^{+}(x) \boldsymbol{\alpha}^{-1}-\boldsymbol{\alpha} \boldsymbol{h}^{-}(x) \boldsymbol{\alpha}^{-1}\right] \boldsymbol{q}
$$

is for a homogeneous slab with a uniform source in the top group and zero flux at the boundaries. This solution was solved on the CPU using MATLAB's built in matrix functions requiring 61s for 101 spatial edits and 256 groups for U238. Next, a Taylor series form of the matrix functions was run on the CPU requiring $44 \mathrm{~s}$. Finally, the same method on the GPU took $20 \mathrm{~s}$. Thus, we see a speedup of a factor of two. We had expected a factor of 20 to 100 . We have now purchased a second GPU, which should give us the performance we expect.

\subsection{Transport theory}

\section{(A14.MGCSN)}

Since extending diffusion theory to the panel formalism has proven to be elusive, it is quite apparent that doing the same for transport theory will prove equally, if not more, elusive. This section, therefore, is included since, in the future, by incorporating HPC, it should be possible to apply the panel format to both transport and diffusion theory. If true, then a multigroup transport theory capability will be required and this is what we now present. Note that much of this work originated before the start of the grant, but has been continually updated.

Two case are considered. The first is new concept in discrete ordinates algorithms to determine the multiplication factor for a 1D nuclear reactor is presented. The algorithm uses Richardsons and $W$-e accelerators (§II.2.a2) to accelerate a 1D multigroup $S n$ solution and eigenvalue to their continuum limits relative to space and angular discretization. The feasibility of the MultiGroup Converged Sn (MGCSn) method is demonstrated on several criticality benchmarks found in the literature as well as for an infinite lattice. 
The second application is a Fourier transform inversion for infinite medium transport and indicates the essence and potential of the multipanel approach.

\section{a. Determination of the multiplication factor $k$}

The multigroup transport equation to be solved in vector form in a homogeneous medium is

$$
\left[\mu \frac{\partial}{\partial x} \boldsymbol{I}+\boldsymbol{\Sigma}\right] \boldsymbol{\phi}(x, \mu)=\frac{1}{2} \sum_{l=0}^{L} P_{l}(\mu) \boldsymbol{\Sigma}_{s l} \boldsymbol{\phi}(x)+\frac{1}{2} \chi \boldsymbol{v} \boldsymbol{\Sigma}_{f}^{T} \boldsymbol{\phi}_{l}(x)
$$

$\phi(x, \mu)$ is the group angular flux vector for $G$-groups and the Legendre moments are the group vectors

$$
\phi(x) \equiv \int_{-1}^{1} d \mu^{\prime} P_{l}\left(\mu^{\prime}\right) \phi\left(x, \mu^{\prime}\right) .
$$

$\chi$ is the vector for the fission spectrum and $\boldsymbol{v} \boldsymbol{\Sigma}_{f}$ is the vector $\left\{v_{g} \Sigma_{f g}\right\}$. Initially, we assume zero incoming current

$$
\begin{aligned}
& \phi(0, \mu)=0 \\
& \phi(a,-\mu)=0 .
\end{aligned}
$$

From discretization in the spatial and angular variables, the discretized transport equation to solve by sweeping across the slab, first in the positive directions and then the negative directions, is for $\mu_{m}>0$

$$
\boldsymbol{\phi}_{m, j+1}^{k+1}=\left[\boldsymbol{\mu}_{m} \boldsymbol{I}+\frac{h}{2} \boldsymbol{\Sigma}_{j}\right]^{-1}\left\{\left[\boldsymbol{\mu}_{m} \boldsymbol{I}-\frac{h}{2} \boldsymbol{\Sigma}_{j}\right] \boldsymbol{\phi}_{m j}^{k+1}+\frac{h}{2}\left[\boldsymbol{q}_{m, j+1}^{k}+\boldsymbol{q}_{m j}^{k}\right]\right\},
$$

and for $\mu_{m}<0$

$$
\boldsymbol{\phi}_{m, j+1}^{k+1}=\left[\boldsymbol{\mu}_{m} \boldsymbol{I}-\frac{h}{2} \boldsymbol{\Sigma}_{j}\right]^{-1}\left\{\left[\boldsymbol{\mu}_{m} \boldsymbol{I}+\frac{h}{2} \boldsymbol{\Sigma}_{j}\right] \boldsymbol{\phi}_{m j}^{k+1}-\frac{h}{2}\left[\boldsymbol{q}_{m, j+1}^{k}+\boldsymbol{q}_{m j}^{k}\right]\right\},
$$

where the collision source, 


$$
\boldsymbol{q}_{m j}^{k} \equiv \frac{1}{2} \sum_{l=0}^{L} P_{l}\left(\mu_{m}\right) \boldsymbol{\Sigma}_{s l} \boldsymbol{\phi}_{, j}^{k}+\frac{1}{2} \chi_{j} \boldsymbol{v} \boldsymbol{\Sigma}_{f j}^{T} \boldsymbol{\phi}_{0}(x),
$$

is lagged. The slab is discretized and we apply half-range Gauss quadrature abscissae. From integration over all directions and summing over all energy groups, there results for the multiplication factor

$$
k=\frac{\sum_{j=1}^{n} \boldsymbol{e}^{T} \mathrm{~g} \chi_{j} \boldsymbol{v} \boldsymbol{\Sigma}_{f j}^{T} \int_{x_{j}}^{x_{j+1}} d x \phi(x)}{\left[\boldsymbol{e}^{T} \mathrm{~g}\left[\boldsymbol{J}^{+}(a)+\boldsymbol{J}^{-}(0)\right]+\sum_{j=1}^{n} \boldsymbol{e}^{T} \mathrm{~g}\left(\boldsymbol{\Sigma}_{j}-\boldsymbol{\Sigma}_{s j}\right) \int_{x_{j}}^{x_{j+1}} d x \boldsymbol{\phi}(x)\right]} .
$$

For the sum over energy groups, we have taken the inner product with respect to

$$
\boldsymbol{e} \equiv\{1, g=1, \ldots, G\} .
$$

Other possibilities of forming a scalar equation for $k$ exist like including a weighting factor, possibly the flux itself.

All results to follow are found by applying either Richardsons or $W$-e acceleration to the iteration of $\operatorname{Eqs}(3)$ and (4) formed by reducing $h$ by a factor of 2 and increasing $n$ by two until convergence.

\section{a1. Preliminary results}

\section{a1.1 Single homogeneous medium: Few-group}

Here, we consider only a single homogeneous medium. In Ref. [7], there are three

Table 1

Single homogeneous medium two-group cases from Ref. [7]

\begin{tabular}{|c|c|c|c|c|}
\hline $\begin{array}{l}1 \\
2\end{array}$ & $\begin{array}{l}0.2208 \\
0.3360\end{array}$ & $\begin{array}{c}0.0936 \\
0.08544\end{array}$ & $\begin{array}{l}3.10 \\
2.93\end{array}$ & $\begin{array}{l}0.575 \\
0.425\end{array}$ \\
\hline \multicolumn{5}{|c|}{ Transfer cross section matrix } \\
\hline $\mathrm{g}, \mathrm{g}^{\prime}$ & & 1 & & 2 \\
\hline 1 & & 0.0792 & & 0.0432 \\
\hline 2 & & 0.0 & & 0.23616 \\
\hline
\end{tabular}

\section{Case 1}

$x_{c}=1.7956023 \mathrm{~cm}$

Case 2 


\begin{tabular}{|c|c|c|c|c|}
\hline \multicolumn{5}{|c|}{$x_{c}=3.006375 \mathrm{~cm}$} \\
\hline 1 & 0.2160 & 0.06192 & 2.70 & 0.575 \\
\hline 2 & 0.3456 & 0.06912 & 2.50 & 0.425 \\
\hline \multicolumn{5}{|c|}{ Transfer cross section matrix } \\
\hline \multicolumn{2}{|c|}{$\mathrm{g}, \mathrm{g}^{\prime}$} & 1 & \multicolumn{2}{|r|}{2} \\
\hline \multicolumn{2}{|c|}{1} & 0.07824 & \multicolumn{2}{|r|}{0.0720} \\
\hline \multicolumn{2}{|c|}{2} & 0.0 & \multicolumn{2}{|r|}{0.26304} \\
\hline \multicolumn{5}{|c|}{ Case 3} \\
\hline 1 & 0.268165 & 0.15024 & 0.0 & 1.0 \\
\hline 2 & 1.276976 & 0.060706 & 2.830023 & 0.0 \\
\hline \multicolumn{5}{|c|}{ Transfer cross section matrix } \\
\hline \multicolumn{2}{|c|}{$\mathrm{g}, \mathrm{g}^{\prime}$} & 1 & \multicolumn{2}{|r|}{2} \\
\hline \multirow{2}{*}{\multicolumn{2}{|c|}{$\begin{array}{l}1 \\
2\end{array}$}} & 0.247516 & \multicolumn{2}{|r|}{0.020432} \\
\hline & & 0.0 & \multicolumn{2}{|r|}{1.213127} \\
\hline
\end{tabular}

Table 2

Converged results

for homogeneous medium/two-groups: Case 1

\begin{tabular}{cccc}
\hline $\boldsymbol{n}$ & $\boldsymbol{N}$ & Original & $\boldsymbol{W}-\boldsymbol{e}$ \\
\hline 1 & 36 & $8.29692 \mathrm{E}-01$ & $8.29692 \mathrm{E}-01$ \\
2 & 38 & $9.63041 \mathrm{E}-01$ & $9.63041 \mathrm{E}-01$ \\
4 & 40 & $9.90394 \mathrm{E}-01$ & $9.97453 \mathrm{E}-01$ \\
8 & 42 & $9.97562 \mathrm{E}-01$ & $1.00011 \mathrm{E}+00$ \\
16 & 44 & $9.99388 \mathrm{E}-01$ & $1.00001 \mathrm{E}+00$ \\
32 & 46 & $9.99847 \mathrm{E}-01$ & $1.00000 \mathrm{E}+00$ \\
64 & 48 & $9.99962 \mathrm{E}-01$ & $1.00000 \mathrm{E}+00$ \\
128 & 50 & $9.99991 \mathrm{E}-01$ & $1.00000 \mathrm{E}+00$ \\
256 & 52 & $9.99998 \mathrm{E}-01$ & $1.00000 \mathrm{E}+00$ \\
512 & 54 & $1.00000 \mathrm{E}+00$ & $1.00000 \mathrm{E}+00$ \\
\hline
\end{tabular}

Converged results

for homogeneous/two-groups: Case 2

\begin{tabular}{llcc}
\hline$n$ & $N$ & Original & $W-e$ \\
\hline 1 & 12 & $7.75386 \mathrm{E}-01$ & $7.75386 \mathrm{E}-01$ \\
2 & 14 & $9.50168 \mathrm{E}-01$ & $9.50168 \mathrm{E}-01$ \\
4 & 16 & $9.87115 \mathrm{E}-01$ & $9.97019 \mathrm{E}-01$ \\
8 & 18 & $9.96732 \mathrm{E}-01$ & $1.00012 \mathrm{E}+00$ \\
16 & 20 & $9.99179 \mathrm{E}-01$ & $1.00002 \mathrm{E}+00$ \\
32 & 22 & $9.99795 \mathrm{E}-01$ & $9.99998 \mathrm{E}-01$ \\
64 & 24 & $9.99949 \mathrm{E}-01$ & $1.00000 \mathrm{E}+00$ \\
128 & 26 & $9.99987 \mathrm{E}-01$ & $1.00000 \mathrm{E}+00$ \\
256 & 28 & $9.99997 \mathrm{E}-01$ & $1.00000 \mathrm{E}+00$ \\
512 & 30 & $9.99999 \mathrm{E}-01$ & $1.00000 \mathrm{E}+00$ \\
\hline
\end{tabular}


Converged results

for homogeneous two-groups: Case 3

\begin{tabular}{cccc}
\hline$n$ & $N$ & Original & $W-e$ \\
\hline 1 & 12 & $3.44776 \mathrm{E}-01$ & $3.44776 \mathrm{E}-01$ \\
2 & 14 & $8.63737 \mathrm{E}-01$ & $8.63737 \mathrm{E}-01$ \\
4 & 16 & $9.65425 \mathrm{E}-01$ & $9.90206 \mathrm{E}-01$ \\
8 & 18 & $9.91271 \mathrm{E}-01$ & $1.00008 \mathrm{E}+00$ \\
16 & 20 & $9.97807 \mathrm{E}-01$ & $1.00002 \mathrm{E}+00$ \\
32 & 22 & $9.99456 \mathrm{E}-01$ & $1.00001 \mathrm{E}+00$ \\
64 & 24 & $9.99869 \mathrm{E}-01$ & $1.00000 \mathrm{E}+00$ \\
128 & 26 & $9.99973 \mathrm{E}-01$ & $1.00001 \mathrm{E}+00$ \\
256 & 28 & $9.99999 \mathrm{E}-01$ & $1.00001 \mathrm{E}+00$ \\
512 & 30 & $1.00001 \mathrm{E}+00$ & $1.00001 \mathrm{E}+00$ \\
\hline
\end{tabular}

relevant two-group cases shown in Table 1 for determination of the critical dimension $\left(x_{c}\right)$, where $k$ has been set to unity to specify a critical system. The results from the MGCSn code are given in Table 2 indicating exact agreement to these benchmarks at the final iteration for a requested $10^{-10}$ relative error. The ability of the $W$-e algorithm to enhance convergence is demonstrated since fiveplace convergence is achieved (shaded row), where the un-accelerated $k$ has converged only to three-places. Figure 1 shows the fluxes for these cases.

\section{a1.2. Heterogeneous medium}

The final demonstration concerns a four region/four group heterogeneous core/reflector medium. Table 3 gives the nuclear properties for the core and reflector. Each region is $30.48 \mathrm{~cm}$ in width. A multiplication factor of 1.246728 , requiring more than 100 inner iterations per acceleration and completing in about seven accelerations, was found. The number of inner iteration seems excessive, but not so, when one realizes that the code has yet to be optimized and these results are entirely preliminary. The eigenflux is shown in Fig. 2a normalized to the number of fission neutrons. This core was also modeled previously with diffusion theory [6], where the multiplication factor was found to be 1.246368 , which is in relatively close to the transport result. This should be expected since the reactor is large. 


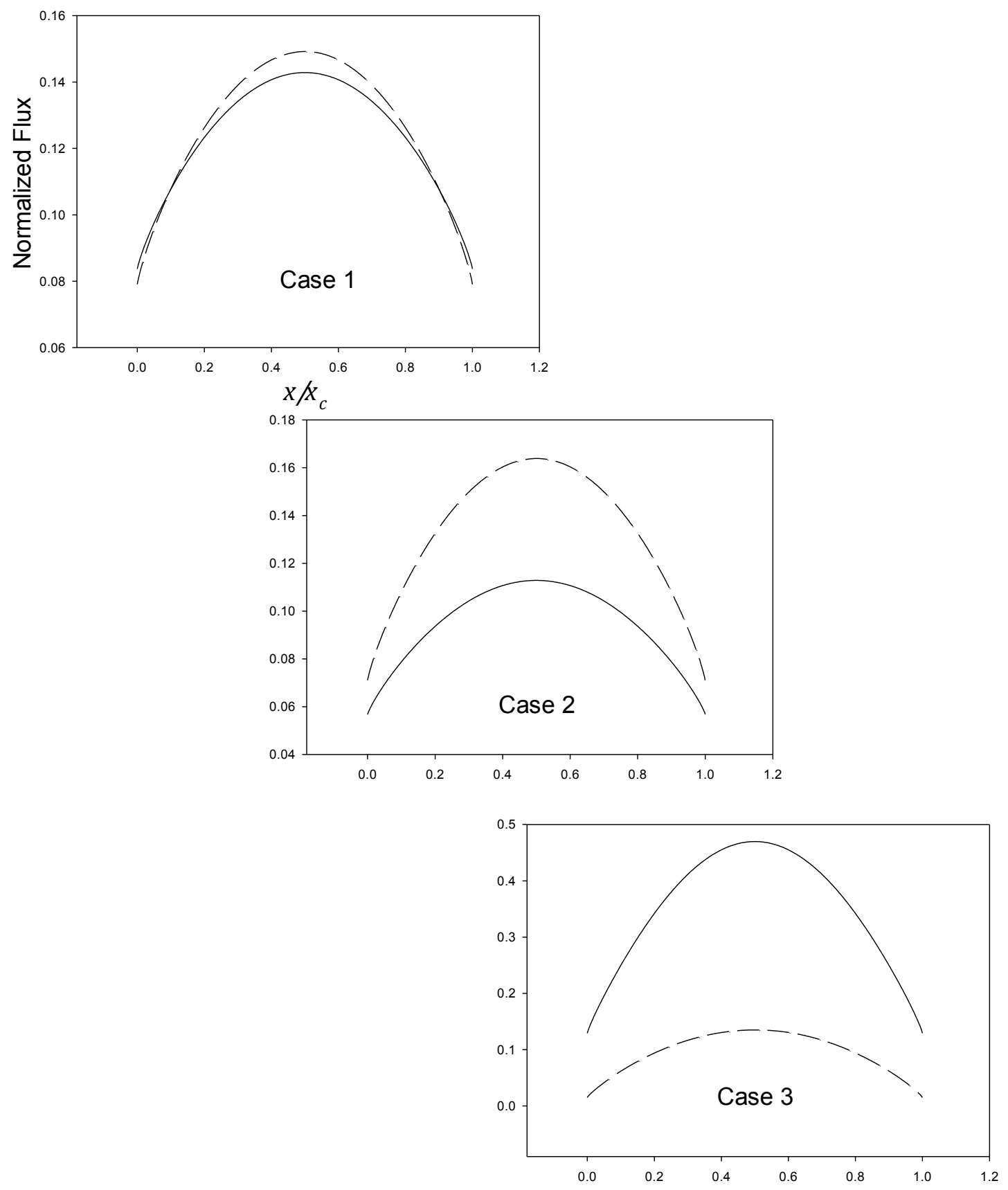

Fig. 1. Normalized fluxes for homogeneous 2- group benchmarks. 
Table 3

Nuclear properties for 4-group/2-region reactor Fuel

\begin{tabular}{|c|c|c|c|c|c|c|c|c|c|}
\hline$g$ & \multicolumn{2}{|c|}{ Sigt } & \multicolumn{2}{|c|}{ (Nu)Sigf } & \multicolumn{2}{|l|}{ Sigr } & \multicolumn{2}{|c|}{$n u$} & Chi \\
\hline 1 & \multicolumn{2}{|c|}{$2.4449 \mathrm{E}+00$} & \multicolumn{2}{|c|}{$9.7350 \mathrm{E}-04$} & \multicolumn{2}{|c|}{$5.89386 \mathrm{E}-02$} & \multicolumn{2}{|c|}{$1.0000 \mathrm{E}+00$} & $7.3760 \mathrm{E}-01$ \\
\hline 2 & \multicolumn{2}{|c|}{$1.2272 \mathrm{E}+00$} & \multicolumn{2}{|c|}{$1.1530 \mathrm{E}-03$} & \multicolumn{2}{|c|}{$6.72016 \mathrm{E}-02$} & \multicolumn{2}{|c|}{$1.0000 \mathrm{E}+00$} & $2.6220 \mathrm{E}-01$ \\
\hline 3 & \multicolumn{2}{|c|}{$1.0764 \mathrm{E}+00$} & 1.7560 & E-02 & \multicolumn{2}{|c|}{$7.30780 \mathrm{E}-02$} & \multicolumn{2}{|c|}{$1.0000 \mathrm{E}+00$} & $2.0000 \mathrm{E}-04$ \\
\hline 4 & \multicolumn{2}{|c|}{$3.7650 \mathrm{E}-01$} & 2.3800 & E-01 & \multicolumn{2}{|c|}{$1.75600 \mathrm{E}-01$} & \multicolumn{2}{|c|}{$1.0000 \mathrm{E}+00$} & $0.0000 \mathrm{E}+00$ \\
\hline & \multicolumn{9}{|c|}{ Transfer xsecs } \\
\hline & 1 & \multicolumn{2}{|c|}{$0.000000 \mathrm{E}+00$} & \multicolumn{2}{|c|}{$5.824000 \mathrm{E}-02$} & \multirow{2}{*}{\multicolumn{2}{|c|}{$0.000000 \mathrm{E}+00$}} & \multicolumn{2}{|c|}{$0.000000 \mathrm{E}+00$} \\
\hline & 2 & \multirow{2}{*}{\multicolumn{2}{|c|}{$0.000000 \mathrm{E}+00$}} & \multicolumn{2}{|c|}{$0.000000 \mathrm{E}+00$} & & & \multicolumn{2}{|c|}{$0.000000 \mathrm{E}+00$} \\
\hline & 3 & & & \multicolumn{2}{|c|}{$0.000000 \mathrm{E}+00$} & \multicolumn{2}{|c|}{ 6.644200E-02 } & \multicolumn{2}{|c|}{$5.751800 \mathrm{E}-02$} \\
\hline & 4 & \multicolumn{2}{|c|}{$\begin{array}{l}0.000000 \mathrm{E}+00 \\
0.000000 \mathrm{E}+00\end{array}$} & \multicolumn{2}{|c|}{$0.000000 \mathrm{E}+00$} & \multicolumn{2}{|c|}{$0.000000 \mathrm{E}+00$} & \multicolumn{2}{|c|}{$0.000000 \mathrm{E}+00$} \\
\hline & \multicolumn{9}{|c|}{ Reflector } \\
\hline$g$ & \multicolumn{2}{|c|}{ Sigt } & $(\mathrm{Nu}) \mathrm{Si}$ & & Sigr & & $n u$ & & Chi \\
\hline 1 & 1.624 & $\overline{E+00}$ & $0.0000 \mathrm{I}$ & $E+00$ & 6.2000 & $0 \mathrm{E}-02$ & 0.0000 & $E+00$ & $0.0000 \mathrm{E}+00$ \\
\hline 2 & 6.620 & E-01 & $0.0000 \mathrm{H}$ & $E+00$ & 4.4600 & 0E-02 & 0.0000 & $E+00$ & $0.0000 \mathrm{E}+00$ \\
\hline 3 & 5.000 & E-01 & $0.0000 \mathrm{H}$ & $E+00$ & 3.6500 & 0E-02 & 0.0000 & $\mathrm{E}+00$ & $0.0000 \mathrm{E}+00$ \\
\hline 4 & 3.448 & E-01 & $0.0000 \mathrm{~F}$ & $E+00$ & 3.5000 & OE-03 & 0.0000 & $E+00$ & $0.0000 \mathrm{E}+00$ \\
\hline
\end{tabular}

Transfer xsecs

\begin{tabular}{lllll}
\hline 1 & $0.000000 \mathrm{E}+00$ & $6.930000 \mathrm{E}-02$ & $0.000000 \mathrm{E}+00$ & $0.000000 \mathrm{E}+00$ \\
2 & $0.000000 \mathrm{E}+00$ & $0.000000 \mathrm{E}+00$ & $4.450000 \mathrm{E}-02$ & $0.000000 \mathrm{E}+00$ \\
3 & $0.000000 \mathrm{E}+00$ & $0.000000 \mathrm{E}+00$ & $0.000000 \mathrm{E}+00$ & $3.640000 \mathrm{E}-02$ \\
4 & $0.000000 \mathrm{E}+00$ & $0.000000 \mathrm{E}+00$ & $0.000000 \mathrm{E}+00$ & $0.000000 \mathrm{E}+00$ \\
\hline
\end{tabular}

We now use the above core configuration to demonstrate a lattice cell calculation. With reflective boundary conditions, the multiplication factor of $\mathrm{Eq}(4)$ becomes for a lattice cell

$$
k=\frac{\sum_{j=1}^{n} \boldsymbol{e}^{T} \mathrm{~g} \chi_{j} \boldsymbol{v} \boldsymbol{\Sigma}_{f j}^{T} \int_{x_{j}}^{x_{j+1}} d x \phi(x)}{\left.\sum_{j=1}^{n} \boldsymbol{e}^{T} \mathrm{~g} \boldsymbol{\Sigma}_{j}-\boldsymbol{\Sigma}_{s j}\right) \int_{x_{j}}^{x_{j+1}} d x \boldsymbol{\phi}(x)}
$$

since the boundary current vanishes. Figure $2 \mathrm{~b}$ shows the eigenflux for this case, where the zero current condition at the outer surfaces is clear. $k$ is 1.247781 and compares well with the diffusion theory result [6] of 1.247483 . 


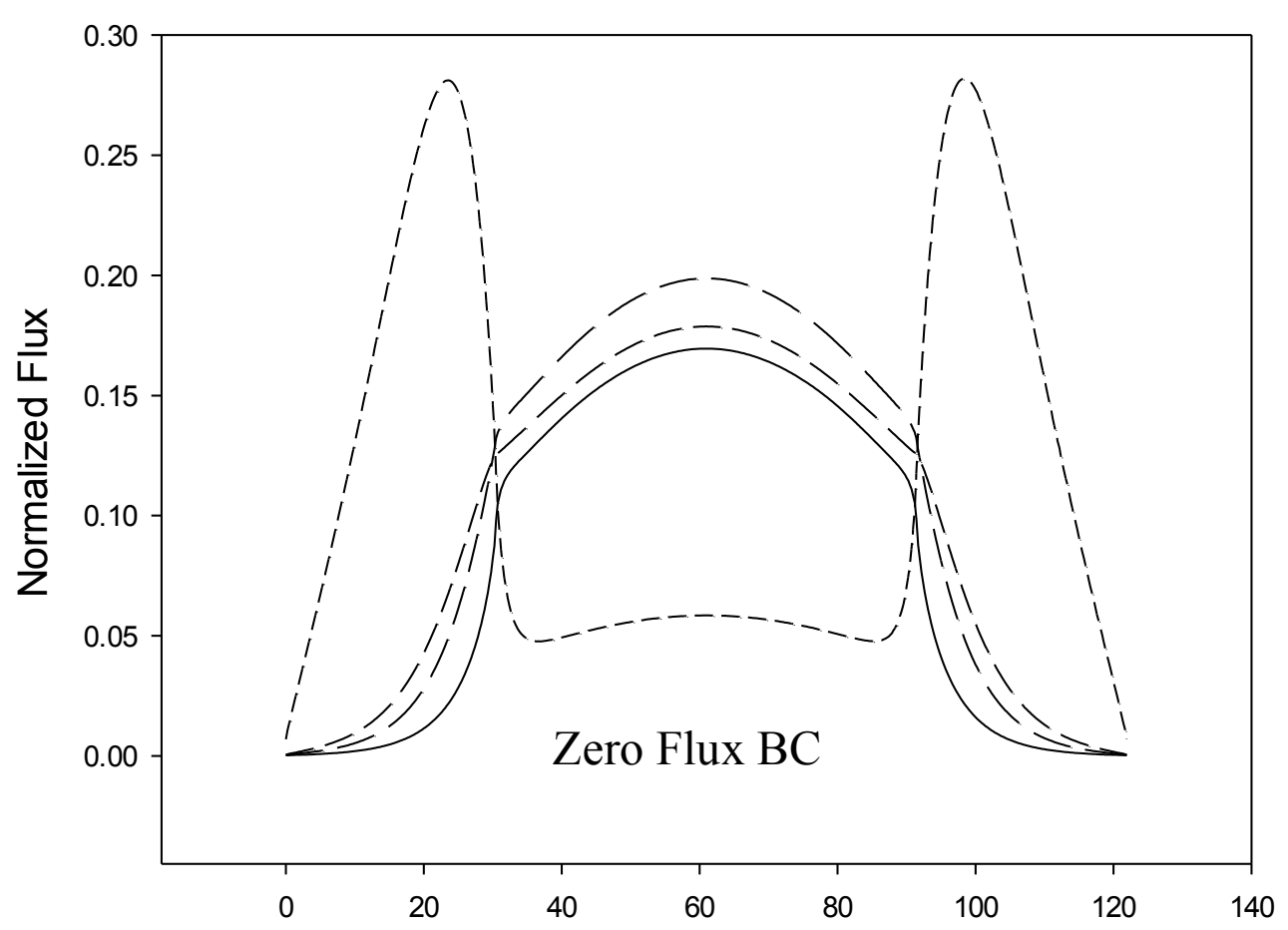

Fig.7a. Eigenflux for the four group/four region model.

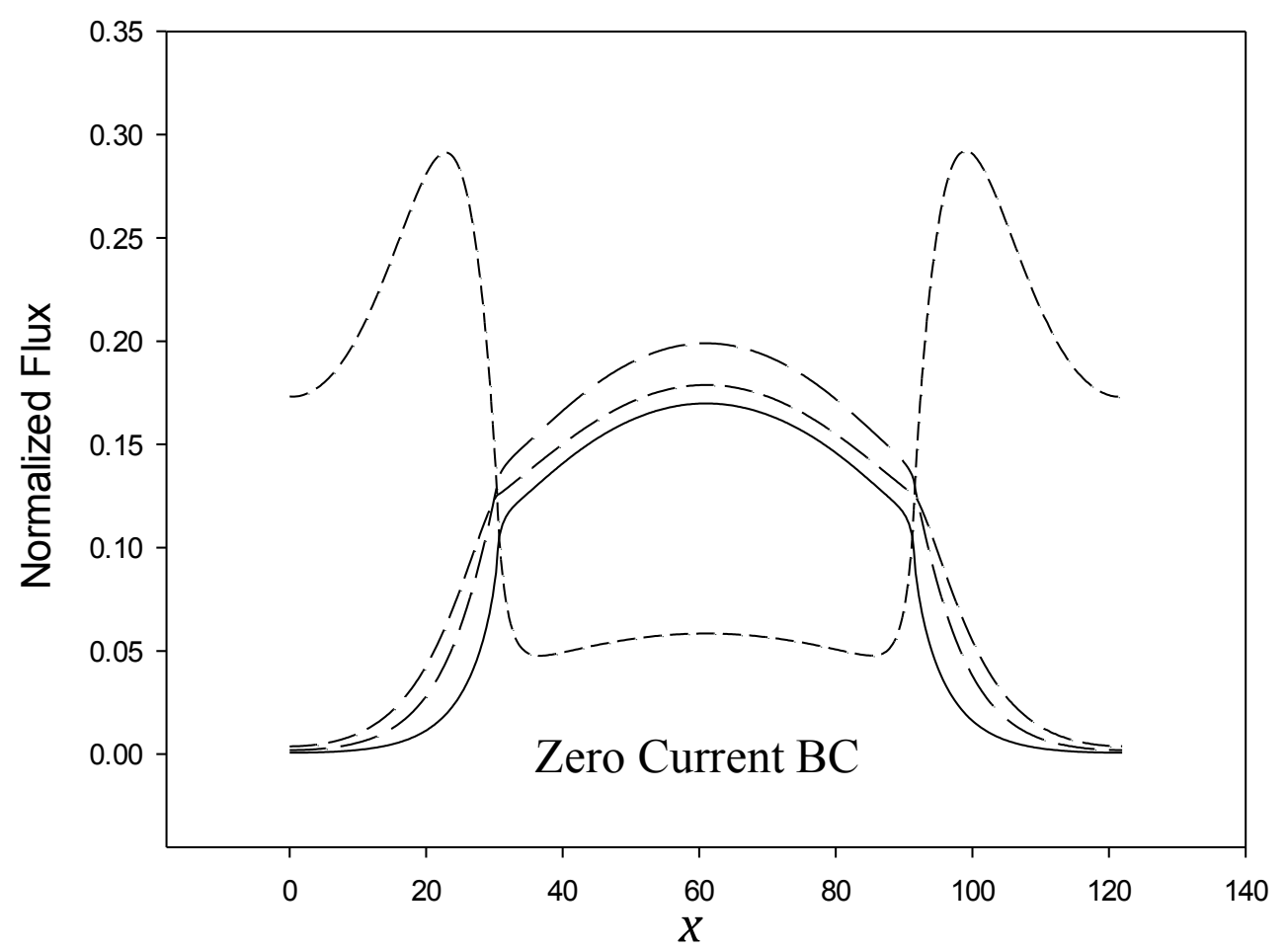

Fig.7b. Eigenflux for the four group/four region model.

Since, with the vanishing of the boundary current, the cell becomes isolated, we 
have an excellent intuitive benchmarking opportunity. We can assemble adjacent unit cells and check if the multiplication factor remains unchanged, which should provide a stringent test of any algorithm. This was done for $2,4,8,16$ cells with the multiplication factor remaining constant to six places as shown in Table 4. The anomaly at two cells is somewhat curious however. If, for this case, each region is further divided in two and the computation repeated, then the two-cell result becomes 1.247780, which compares well with the rest.

As shown in Fig. 3 , the flux for 16 cells behaves intuitively as expected.

Table 4

Multiplication factor for 4-region/4-group lattice cells

\begin{tabular}{cc}
\hline Cells & $k$ \\
\hline 1 & 1.247781 \\
2 & 1.247488 \\
4 & 1.247781 \\
8 & 1.247779 \\
16 & 1.247781 \\
\hline
\end{tabular}

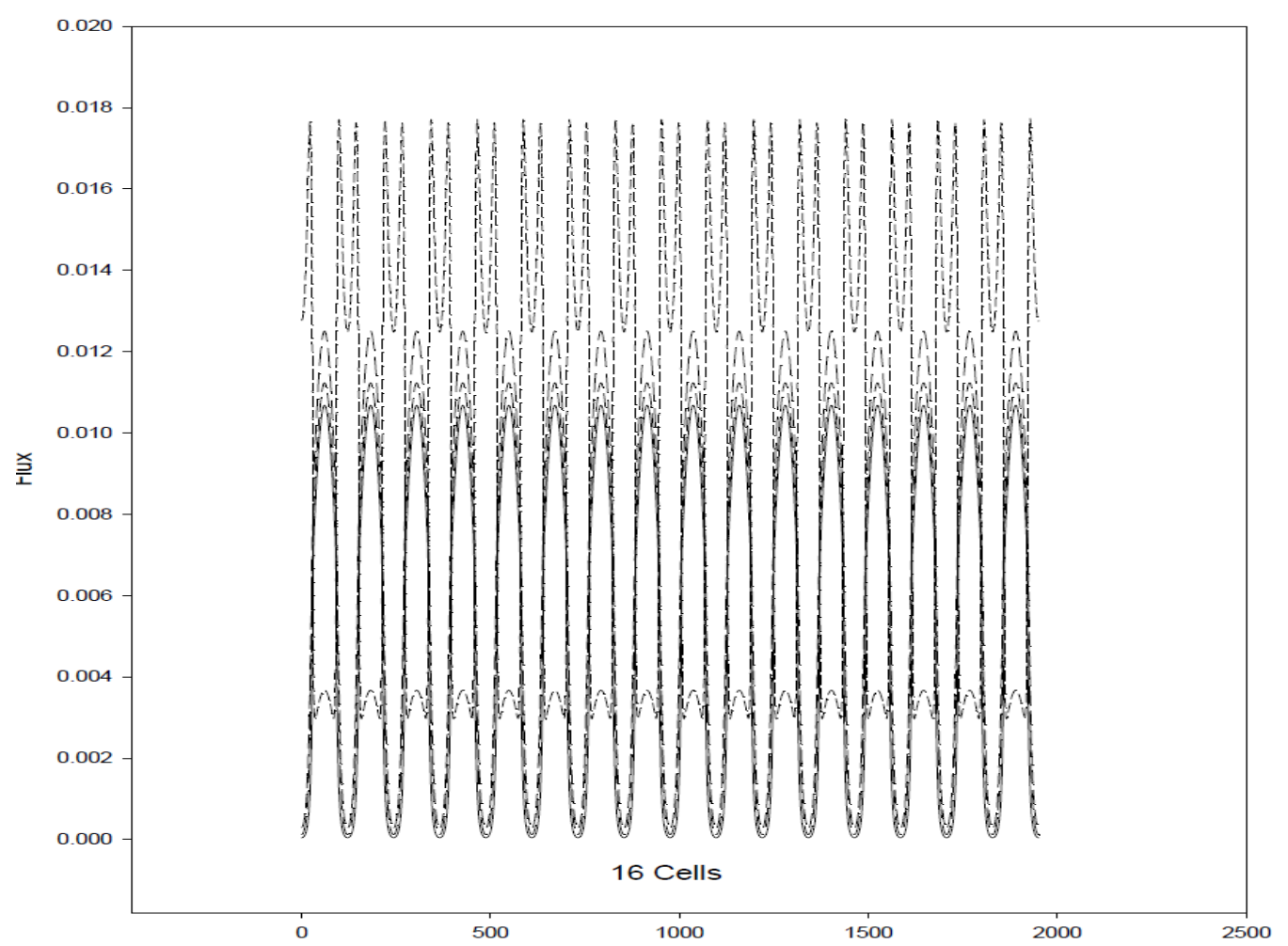

Fig. 3. Infinite lattice of 16 cells. 


\section{b. MG/FG/UfG transport solution in an infinite medium}

MG.FG.Inf.Medium

\section{b1. Single panel solution}

Here, we suggest a possibility for a FG/UfG solution using the panel formalism for slowing down in an 1D infinite plane medium. The presentation will essentially be PPT slides from this past summer's short course developed for INL.

We begin with the MG solution as the precursor to FG/UfG solutions. The multigroup transport equation is shown on the first slide. This is the Green's function in plane geometry. With the substitution shown, the bottom equation is

- Infinite medium MG Green's function (no fission)

- Beam source at center plane in direction $\mu$

$$
\begin{aligned}
& {\left[\mu \underline{\underline{I}} \frac{\partial}{\partial x}+\Sigma\right] \Phi\left(x, \mu, \mu^{\prime}\right)=\frac{1}{2} \sum_{l=0}^{L}(2 l+1) P_{l}(\mu) \Sigma_{s l} \Phi_{l}\left(x, \mu^{\prime}\right)+\delta(\mu-\mu l) \delta(x) \boldsymbol{q}} \\
& \Sigma \equiv \operatorname{diag}\left[\Sigma_{g}, g=1, \ldots, G\right] \\
& \Phi\left(x, \mu ; \mu^{\prime}\right)=\underline{\Psi}\left(x, \mu ; \mu^{\prime}\right) \boldsymbol{q} \\
& {\left[\mu \boldsymbol{I} \frac{\partial}{\partial x}+\Sigma\right] \Psi\left(x, \mu, \mu^{\prime}\right)=\frac{1}{2} \sum_{l=0}^{L}(2 l+1) P_{l}(\mu) \Sigma_{s} \Psi_{l}\left(x, \mu^{\prime}\right)+\delta\left(\mu-\mu \mu^{\prime}\right) \delta(x) \boldsymbol{I}} \\
& \quad+\text { In matrix form }
\end{aligned}
$$

Slide 1

- Take a Fourier transform

$$
\Psi\left(k, \mu ; \mu^{\prime}\right) \equiv \int_{-\infty}^{\infty} d x e^{-i k x} \Psi\left(x, \mu ; \mu^{\prime}\right)
$$

+ To give (note similarity to previous)

$$
\begin{aligned}
& {[\Sigma+i k \mu \boldsymbol{I}] \Psi\left(k, \mu ; \mu^{\prime}\right)=} \\
& \quad=\frac{1}{2} \sum_{l=0}^{L}(2 l+1) P_{l}(\mu) \boldsymbol{\Sigma}_{s l} \Psi_{l}\left(k ; \mu^{\prime}\right)+\delta\left(\mu-\mu^{\prime}\right) \boldsymbol{I}
\end{aligned}
$$




\section{Slide 2}

the fundamental matrix equation to be solved in direct space.

In slide 2, a Fourier transform is taken to bring the equation to a matrix set in transform space. Now it is a matter of projection to find the transformed moments as shown in slide 3 . This is the first of two moments equation, which can be solved directly for all required moments.

\section{- First moments eqn}

$$
\begin{gathered}
\sum_{l=0}^{L}\left[\delta_{j l} \boldsymbol{I}-(2 l+1) z \boldsymbol{L}_{j l}(z \Sigma) \Sigma_{s l}\right] \Psi_{l}\left(k ; \mu^{\prime}\right)=z[z \Sigma+\mu \boldsymbol{I}]^{-1} P_{j}\left(\mu^{\prime}\right) \\
\boldsymbol{L}_{j l}(z \Sigma) \equiv\left\{\frac{1}{2} \int_{-1}^{1} d \mu \frac{P_{j}(\mu) P_{l}(\mu)}{z \Sigma-\mu} ; l, j=1, \ldots, G\right\} \\
z \equiv \frac{1}{i k}
\end{gathered}
$$

Slide 3

- Second moments eqn

+ Project transform eqn over $P_{j}(\mu)$

$\int d \mu P_{j}(\mu)[\Sigma+i k \mu \boldsymbol{I}] \Psi\left(k, \mu ; \mu^{\prime}\right)=$

$=\frac{1}{2} \int_{-1}^{1} d \mu P_{j}(\mu) \sum_{l=0}^{L}(2 l+1) P_{l}(\mu) \Sigma_{s l} \Psi_{l}\left(k ; \mu^{\prime}\right)+P_{j}\left(\mu^{\prime}\right) \boldsymbol{I}$

$z \boldsymbol{h}_{j} \Psi_{j}\left(k ; \mu^{\prime}\right)+(j+1) \Psi_{j+1}\left(k ; \mu^{\prime}\right)+j \Psi_{j-1}\left(k ; \mu^{\prime}\right)=$

$$
=(2 j+1) z P_{j}\left(\mu^{\prime}\right) \boldsymbol{I}
$$

$$
\boldsymbol{h}_{j} \equiv(2 l+1)\left(\boldsymbol{\Sigma}-\boldsymbol{\Sigma}_{s l}\right)
$$

Slide 4 
A second moments equation is derived by simply projecting the bottom equation on Slide 2 over Legendre polynomials to give the last equation on Slide 4. This moments equation is not closed; but is a three-term recurrence with the analytical solution shown as the second equation on Slide 4 with the scalar flux $\Psi_{0}\left(k ; \mu^{\prime}\right)$

$$
\begin{gathered}
+ \text { Now } \\
\boldsymbol{S}_{j}\left(z, \mu^{\prime}\right)=(2 j+1) P_{j}\left(\mu^{\prime}\right) \boldsymbol{I} \\
\bullet \text { Implies } \\
\quad \Psi_{j}\left(k ; \mu^{\prime}\right)=\boldsymbol{g}_{l}(z) \Psi_{0}\left(k ; \mu^{\prime}\right)-\boldsymbol{\chi}_{l}\left(z ; \mu^{\prime}\right) \\
\boldsymbol{\chi}_{l}\left(z ; \mu^{\prime}\right)=\boldsymbol{\rho}(z)- \\
-\sum_{j=1}^{l-1}(2 j+1)\left[\boldsymbol{g}_{l}(z) \boldsymbol{g}_{j}^{-1}(z)-\boldsymbol{\rho}_{l}(z) \boldsymbol{\rho}_{j}^{-1}(z)\right] \boldsymbol{W}_{j}^{-1}(z) z P_{j}\left(\mu^{\prime}\right) \\
W_{j}(z) \equiv j\left[\boldsymbol{\rho}_{j-1}(z) \boldsymbol{\rho}_{j}^{-1}(z)-\boldsymbol{g}_{j-1}(z) \boldsymbol{g}_{j}^{-1}(z)\right]
\end{gathered}
$$

\section{Slide 5}

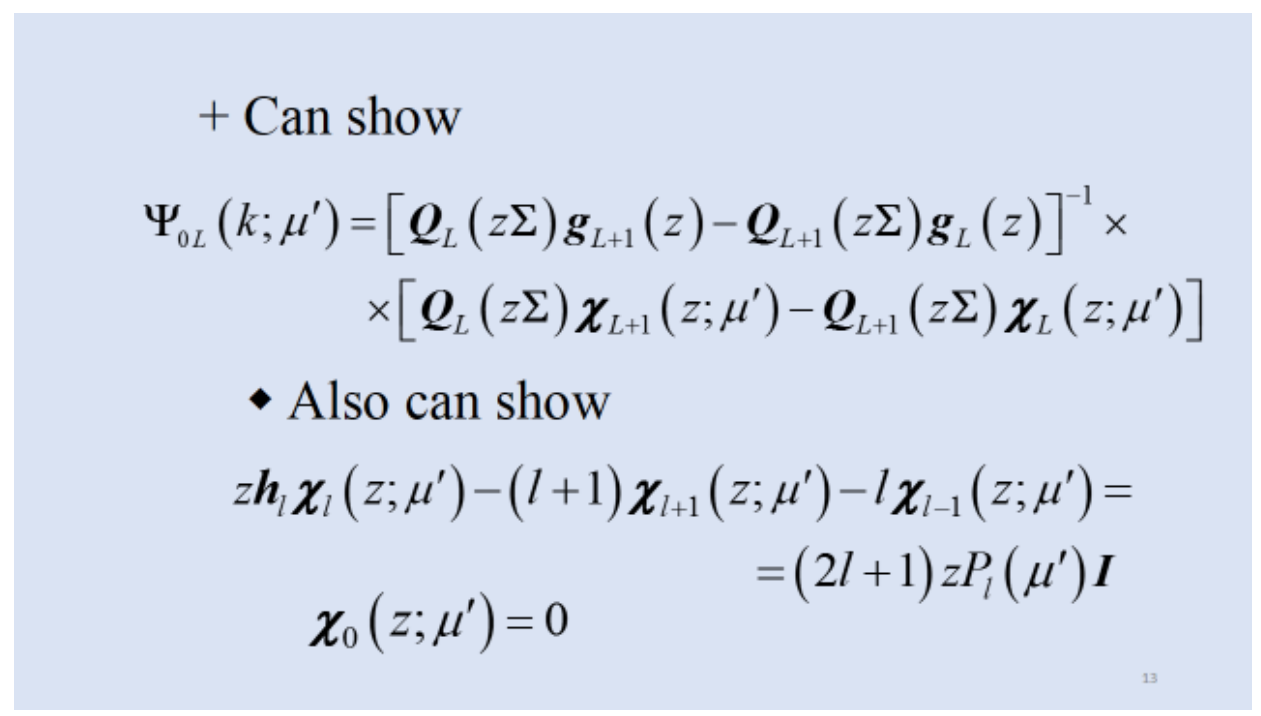

\section{Slide 6}

to be determined. The $\boldsymbol{g}_{l}(z)$ and $\boldsymbol{\rho}_{l}(z)$ are the Chandrasekhar matrices of the first and second kinds respectively. $\Psi_{0}\left(k ; \mu^{\prime}\right)$ is found by substitution of the first moments equation for $j=0$ into the second and, after some algebra, one can 
show the first equation on Slide 6, where $\boldsymbol{Q}_{l}(z \boldsymbol{\Sigma})$ is a diagonal matrix of Legendre functions of the second kind of order $l$.

Finally, we take the inverse Fourier transform shown on Slide 7. As indicated, the inversion will be performed numerically [3], about which more will be said.

+ Take Fourier transform inversion

$$
\Phi\left(x, \mu, \mu^{\prime}\right) \equiv \frac{1}{2 \pi} \int_{-\infty}^{\infty} d k e^{j k} \boldsymbol{\Psi}\left(k, \mu, \mu^{\prime}\right) \boldsymbol{q}
$$

- Follow similar procedure to LT

- Form as an infinite series

- Quadrature for each term

- Accelerate convergence

\section{Slide 7}

\section{b2. FG/UfG transform solution for an isotropic source}

If, in the solution on Slide $8 \boldsymbol{q} \equiv\left\{\delta_{l, 1}, l=1, \ldots, p G\right\}$ and we multiply by $1 / 2$ and integrate over $\mu^{\prime}$, the scalar flux transform vector for isotropic source emission results

$$
\begin{aligned}
\Phi_{0 L}(k)=\left[\boldsymbol{Q}_{L}(z \boldsymbol{\Sigma}) \boldsymbol{g}_{L+1}(z)-\boldsymbol{Q}_{L+1}(z \boldsymbol{\Sigma}) \boldsymbol{g}_{L}(z)\right]^{-1} \times \\
\times\left[\boldsymbol{Q}_{L}(z \boldsymbol{\Sigma}) \rho_{L+1}(z)-\boldsymbol{Q}_{L+1}(z \boldsymbol{\Sigma}) \rho_{L}(z)\right] \boldsymbol{q},
\end{aligned}
$$

which we must invert. This equation is also

$$
\boldsymbol{A}(k) \Phi_{0 L}(k)=\boldsymbol{B}(k) \boldsymbol{q}(k),
$$

where 


$$
\begin{aligned}
\boldsymbol{A}(k) & \equiv\left[\boldsymbol{Q}_{L}(z \boldsymbol{\Sigma}) \boldsymbol{g}_{L+1}(z)-\boldsymbol{Q}_{L+1}(z \boldsymbol{\Sigma}) \boldsymbol{g}_{L}(z)\right] \\
\boldsymbol{B}(k) & \equiv\left[\boldsymbol{Q}_{L}(z \boldsymbol{\Sigma}) \rho_{L+1}(z)-\boldsymbol{Q}_{L+1}(z \boldsymbol{\Sigma}) \rho_{L}(z)\right]
\end{aligned}
$$

Note that for pure down scatter, all the matrices in $\mathrm{Eq}(2)$ are lower triangular and the $\mathrm{Eq}(2 \mathrm{a})$ schematically has the form shown in Slide 8. Horizontal panels have been superimposed on the full lower triangular matrix. Each panel naturally, partitions into a leading triangular matrix along with trailing blocks.

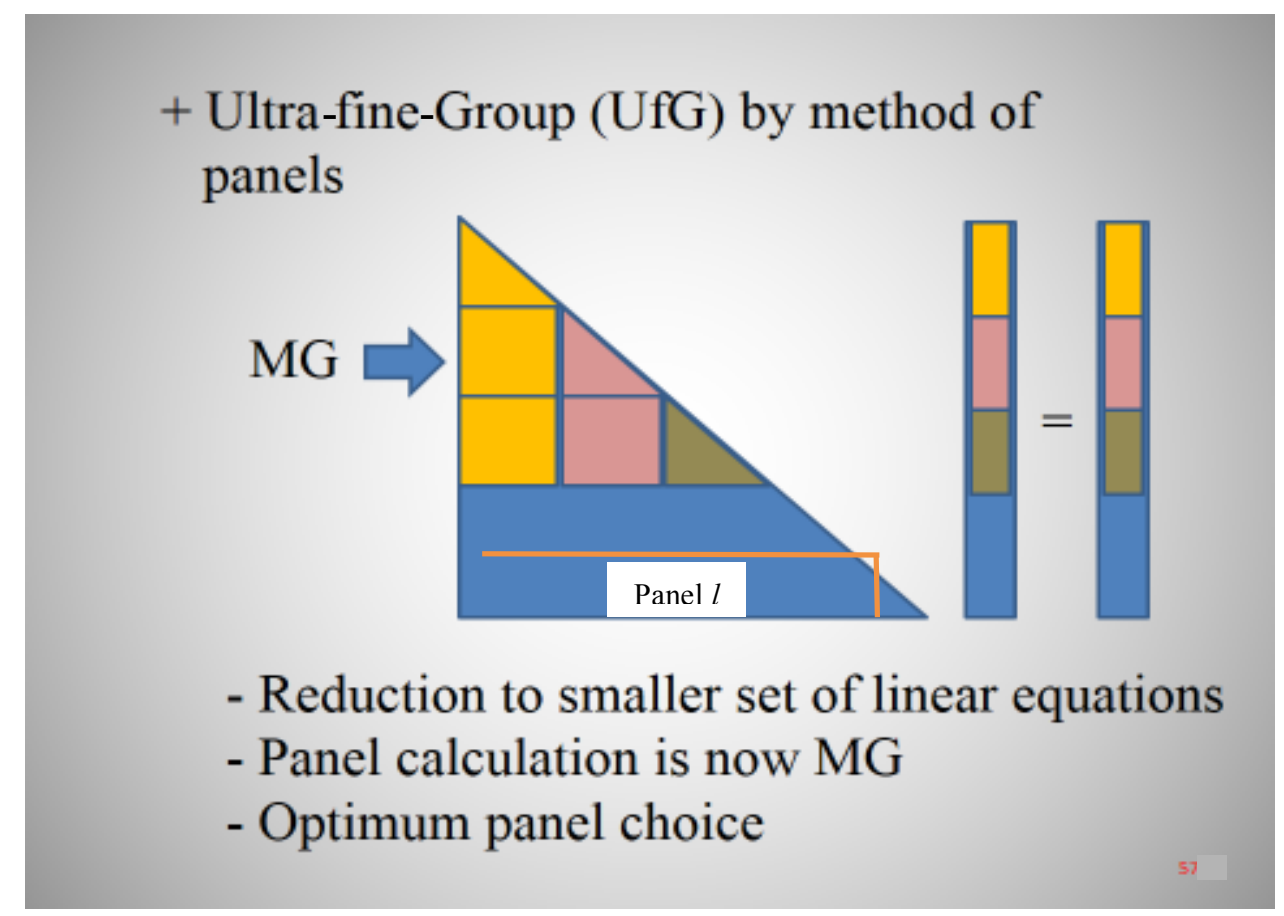

Slide 8

When the flux vector is partitioned into vectors by panel

$$
\Phi_{0 L}(k)=\left[\phi_{1}(k), \phi_{2}(k), \ldots ., \phi_{p}(k)\right]
$$

for $p$ panels each of $G$ groups, the scalar flux in panel $l$ becomes

$$
\boldsymbol{\phi}(k)=\boldsymbol{A}_{l, l}^{-1}(k)\left[(\boldsymbol{B q})_{l}-\sum_{j=1}^{l-1} \boldsymbol{A}_{l, j}(k) \boldsymbol{\phi}_{j}(k)\right] .
$$


$\boldsymbol{A}_{l, j}, j=1, \ldots, l-1$ are the matrix blocks in panel $l$ and $\boldsymbol{A}_{l, l}$ is the leading lower triangular matrix. $(\boldsymbol{B q})_{l}$ is the $l^{\text {th }}$ term on the RHS of $\mathrm{Eq}(2 \mathrm{a})$. This shows the economy of the panel method, where $p \bullet(G x G)$ inversions must be performed rather than a full $p G x p G$ inversion.

\section{b3. FG/UfG transform inversion for an isotropic source}

We now must invert the transform in $\mathrm{Eq}(4)$ to give

$$
\phi(x)=\frac{1}{2 \pi} \int_{-\infty}^{\infty} d k e^{i k x} \phi(k)
$$

for the scalar flux spectral/spatial distribution. The procedure is as follows:

1. A sequence of positive spatial points, $x_{j}$, is chosen.

2. The flux for the first point is determined from the numerical Fourier transform inversion with the integrand $\phi(k)$ saved in the order called by the inversion as it proceeds to completion.

3. The flux for the second point is determined using the stored integrand. If additional integrands are required for convergence of the inversion, they are added to the list in storage.

4. This procedure is repeated for all positions.

In this way, only a minimum of evaluations of $\mathrm{Eq}(4)$ are necessary at the expense of storage.

While this algorithm has yet to be tested, it shows promise at least in the FG application. A conference paper based on this algorithm is currently in progress for the PHYSOR2014 meeting to demonstrate FG. 


\section{II.4. TASK 4 Verification of CENTRM in infinite geometry.}

\section{(A15.ICONE12.paper, A16.ICONE12.Presentation)}

The purpose of this task is to provide a verification the CENTRM (Continuous ENergy TRansport Module) code, an option in the ORNL/SCALE code package, with an entirely independent slowing down transport model. The function of CENTRM is to produce an ultra-fine-group characterization of the neutron spectrum as neutrons slow down to become thermalized. This is the most comprehensive attempt to date to treat neutron absorption in the almostimpossible-to-characterize resolved resonance region. This task is the primary responsibility of UTK guided by Dr. Maldonado with the work performed by graduate student R. Joseph both of whom have access to SCALE and CENTRM as well as the UfG code, X2.f, developed in Task 1.

\subsection{Preliminaries}

\section{a. SCALE cross sections}

To begin the comparison to CENTRM [1], microscopic cross sections (total and scattering) were obtained from the BNL/NNDC according to the prescription given in Task 1. We also obtained the total and scattering cross sections from SCALE [2]. This was done using the modules 'pickeze' and 'charmin' from the AMPX code package (available from RSICC).

The comparison between the microscopic cross sections from BNL and those of SCALE 6.0 for U235 total and scattering are shown in Figs. 1a,b. The energy range is $1000 \mathrm{eV}$ to $2500 \mathrm{eV}$. The comparison indicates that the cross sections are indeed different. This is, to some extent, due to the larger number of the cross sections accessed by SCALE in the range of interest than available from the BNL data. The BNL data has approximately 6,200 data points; whereas, the SCALE data has almost 137,300 points. This inconsistency gives different interpolated values as is observed. In addition, SCALE obtains its data from ENDF/B-VIII-CE (continuous energy); whereas, X2 from ENDF/B-VII, which, because of data, may explain part of the difference, but certainly not all.

The CENTRM module is a part of the SCALE 6.0 package and is usually run in a shielding or criticality study; however, here it is run as a standalone code in order to compare the results to the $\mathbf{X} \mathbf{2}$ code. The particular case to be considered is for a mixture of U235 and graphite. The CENTRM code outputs the flux and macroscopic (total and scattering) as a function of energy. To ensure data consistency, it was confirmed that the CENTRM case was for zero scattering order, no inelastic scattering and $0^{\circ} \mathrm{K}$ temperature. 


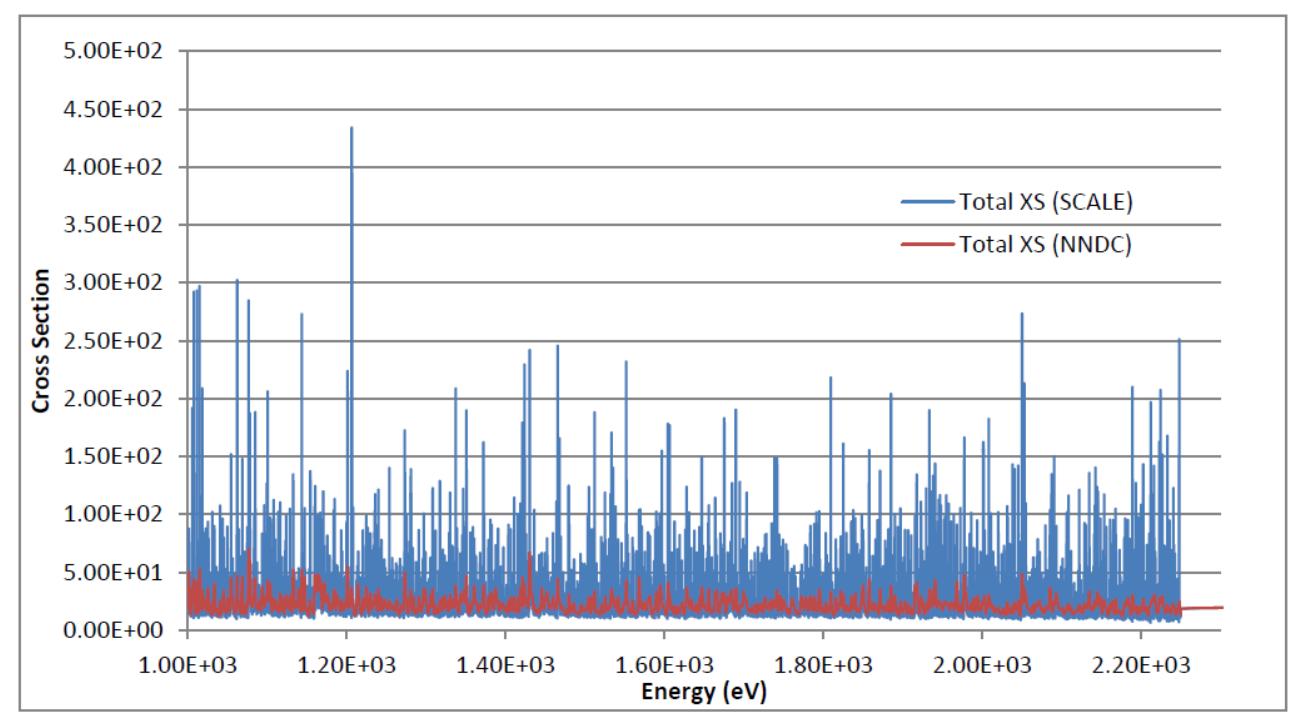

Fig. 1a. Comparison of total microscopic cross sections between SCALE and BNL.

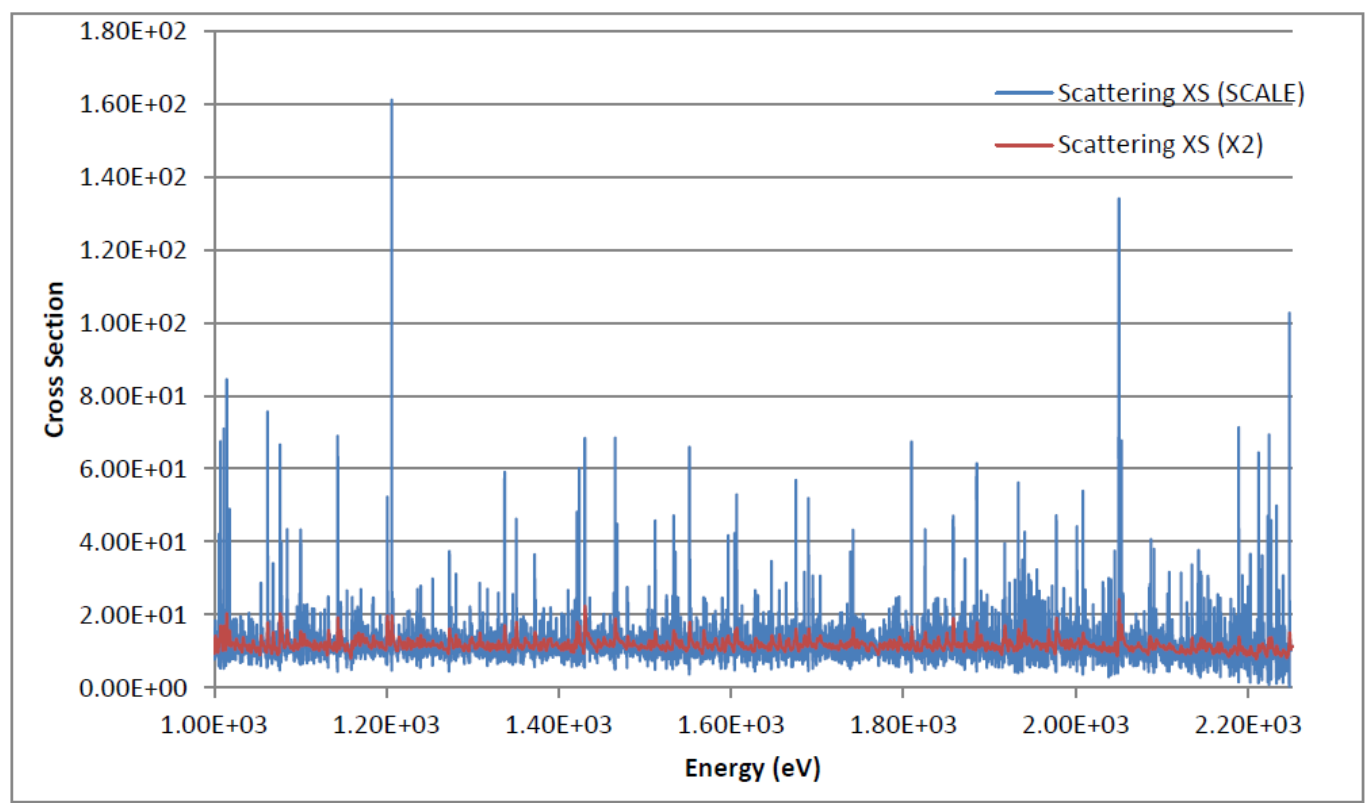

Fig. 1b. Comparison of scattering microscopic cross sections between SCALE and BNL.

\subsection{CENTRM/X2 comparison}

The macroscopic cross sections obtained from CENTRM are shown in Figure 2. For uniformity and to avoid the microscopic cross section discrepancy observed above, the $\mathbf{X} 2$ cross section preparation code ENDFPR was run for the same mixture with the microscopic cross sections generated by SCALE. This ensures that the same cross sections are used as input to both codes. The resulting 
macroscopic cross sections from ENDFPR and CENTRM are compared in Figure 3. The comparison confirms that the ENDFPR and CENTRM codes are now calculating with similar macroscopic cross sections. The slight differences in peaks are due to the different energy edits, where cross sections are recorded in the two codes.

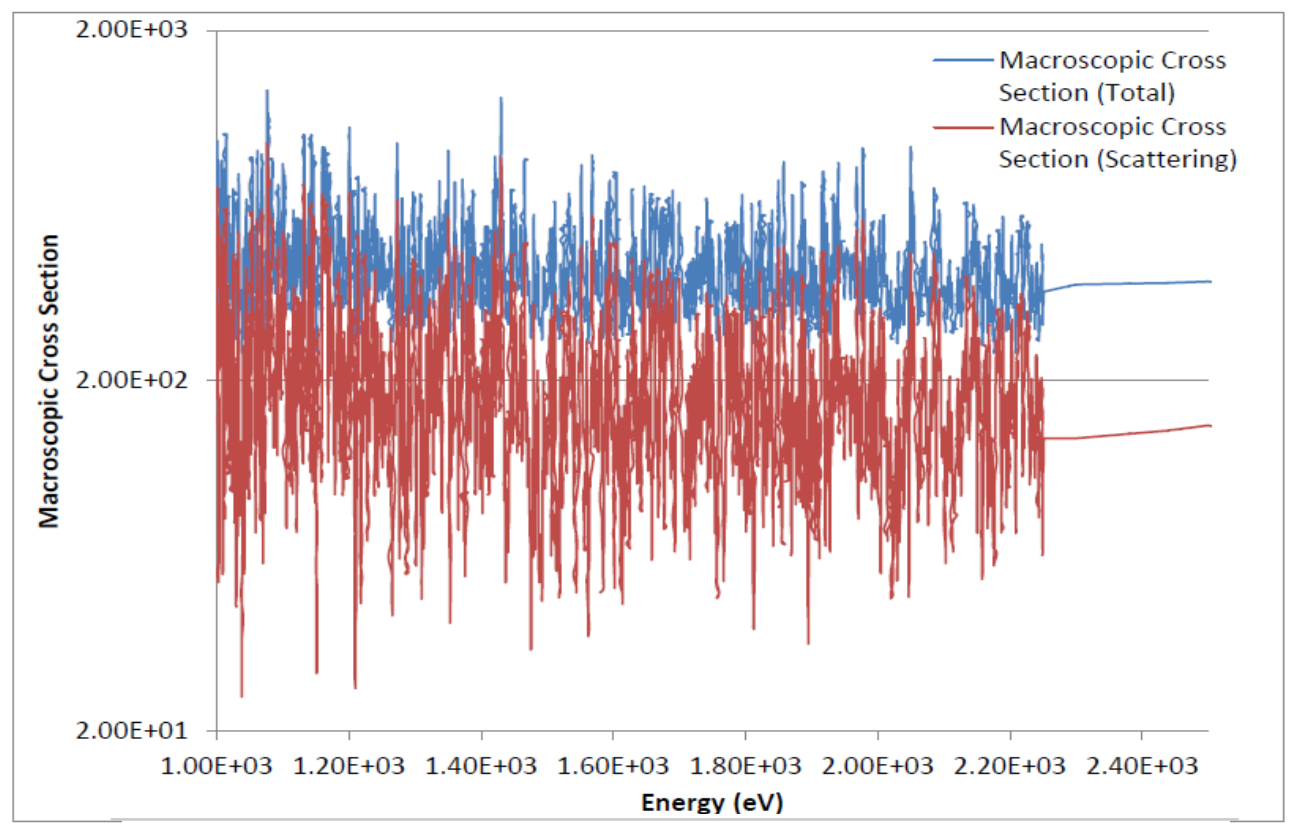

Fig. 2. Macroscopic cross sections from CENTRM.

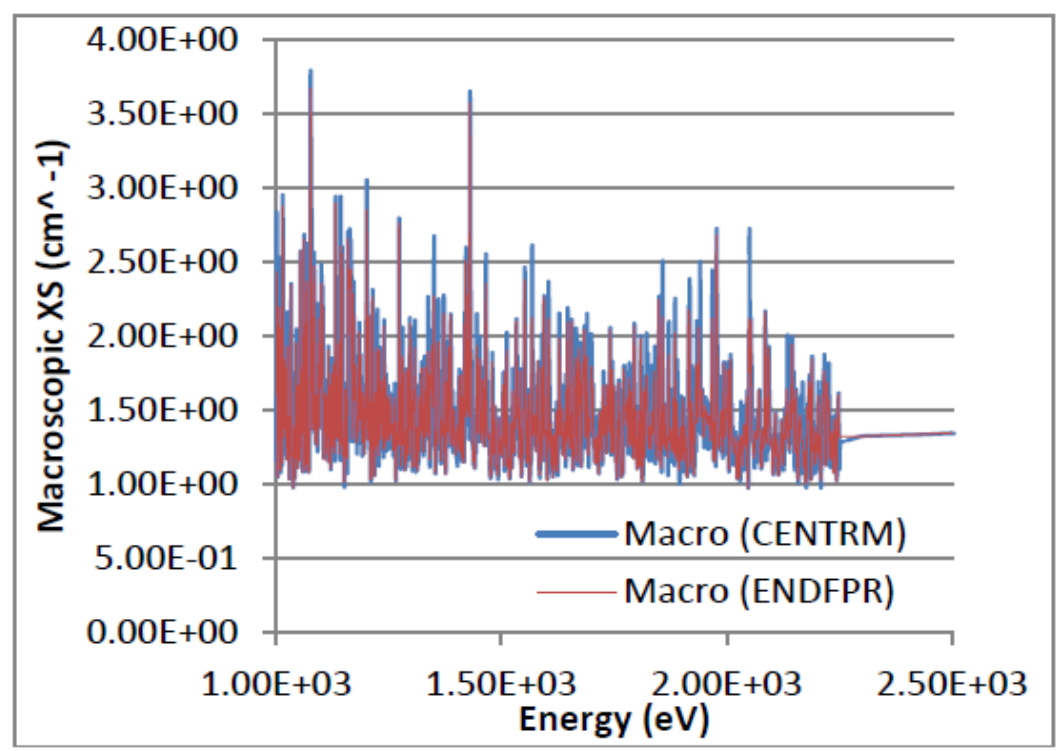

Fig. 3. Comparison of calculated macroscopic cross sections in X2 and CENTRM. 
To provide a further check, a case with just Carbon was simulated with SCALE cross sections in both codes to verify fundamental slowing down. The resulting energy flux (group flux per energy) distribution is shown in Fig. 4 in the range $[1000 \mathrm{eV}, 2500 \mathrm{KeV}]$ indicating the expected $1 / E$ flux variation. Similar $1 / E$ behavior is seen at other energies. The lack of exact agreement is most likely a scale factor due to source normalization. This study shows that CENTRM and X2 are correctly simulating elastic scattering, at least, in Carbon to a source normalization.

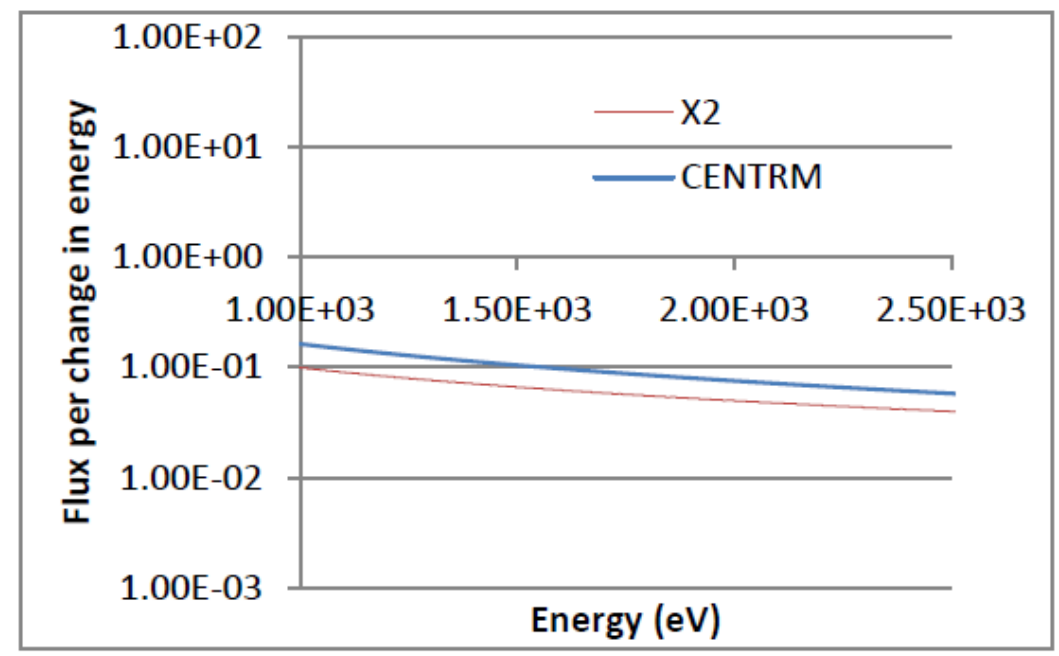

Fig. 4. Comparison of fluxes in X2 and CENTRM for slowing down in Carbon.

X2 with the same mixture of U235 and graphite was run with the cross sections from SCALE. The resulting fluxes from X2 and CENTRM are compared for the energy range $[1000 \mathrm{eV}, 2500 \mathrm{eV}]$ in Figure 5a. Generally, good agreement between the flux distributions is observed. One notable difference is in the way the source is specified giving discrepancy at high energy. Given that the source is specified in a range above $2500 \mathrm{eV}$ in CENTRM [2580e, $3000 \mathrm{eV}$ ] and at a point at $2500 \mathrm{eV}$ in $\mathbf{X} 2$, the data was reported starting at $2200 \mathrm{eV}$ so that the source should not greatly influence the result. In addition, CENTRM's computational methods are more complex that X2's, so slight differences in the results are to be expected.

X2 with the same mixture of U235 and graphite as before, was then run in a lower energy range. This energy range has fewer resonances, and thus should allow the resonances to be more precisely resolved. The resulting flux comparison is given in Fig. $5 \mathrm{~b}$ for the energies between $80 \mathrm{eV}$ and $100 \mathrm{eV}$. Now, we observe very good agreement, but there still seems to be a scale error. 


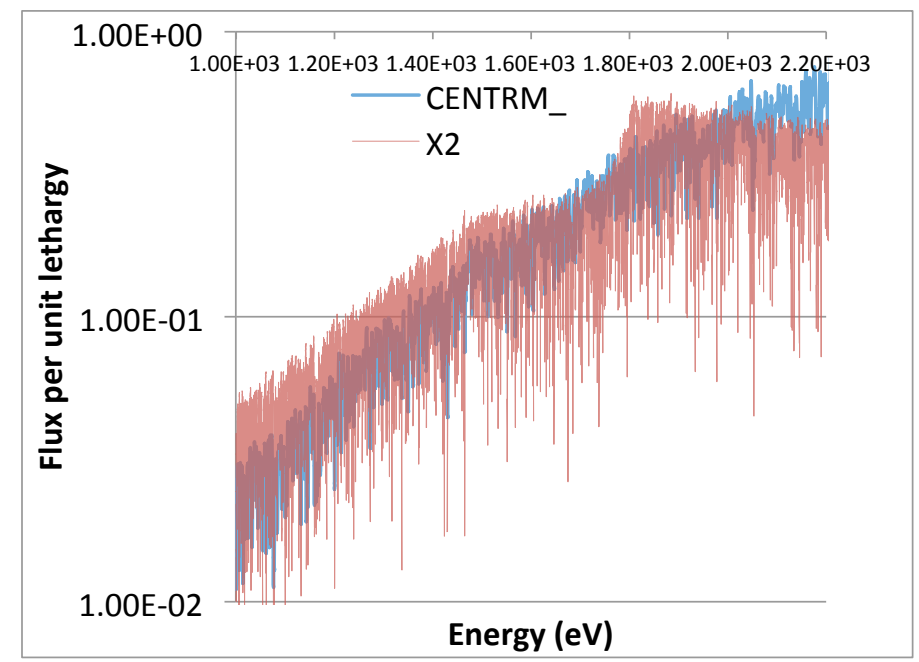

Fig. 5a. Comparison of fluxes in X2 and CENTRM $[1000 \mathrm{eV}, 2200 \mathrm{eV}$.

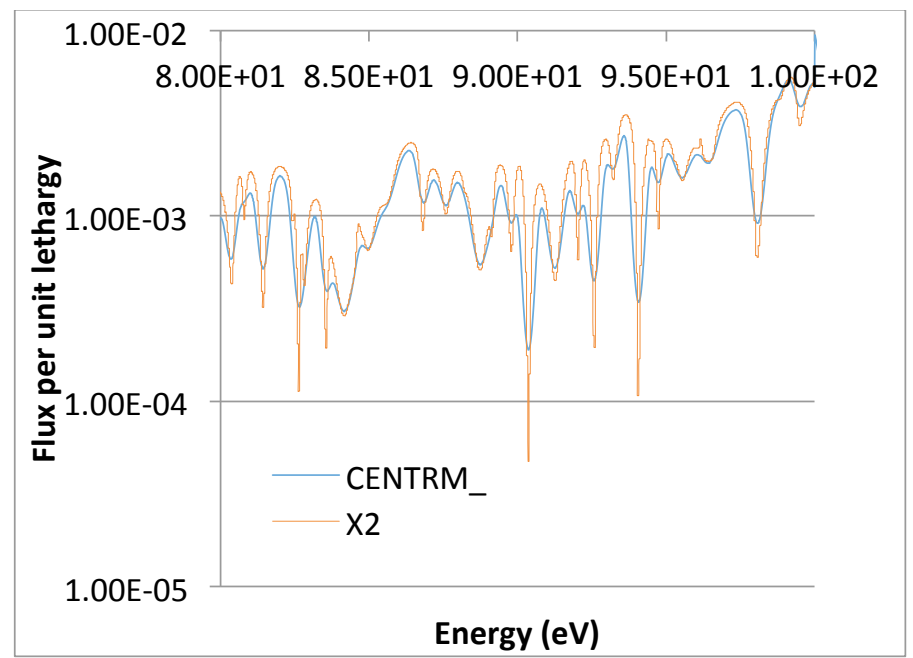

Fig. 5b. Comparison of fluxes in X2 and CENTRM $[80 \mathrm{eV}, 100 \mathrm{eV}]$.

In an attempt to improve upon the results, the source energy was made similar in both codes by a slight modification to the $\mathbf{X} \mathbf{2}$ code. Because there is no way to change the source energy in CENTRM, the X2 code was modified so that the source group could be specified by the user. For this case, the source was placed between $2580 \mathrm{eV}$ and $3000 \mathrm{eV}$. The improved results are shown below in Fig. 6a. 


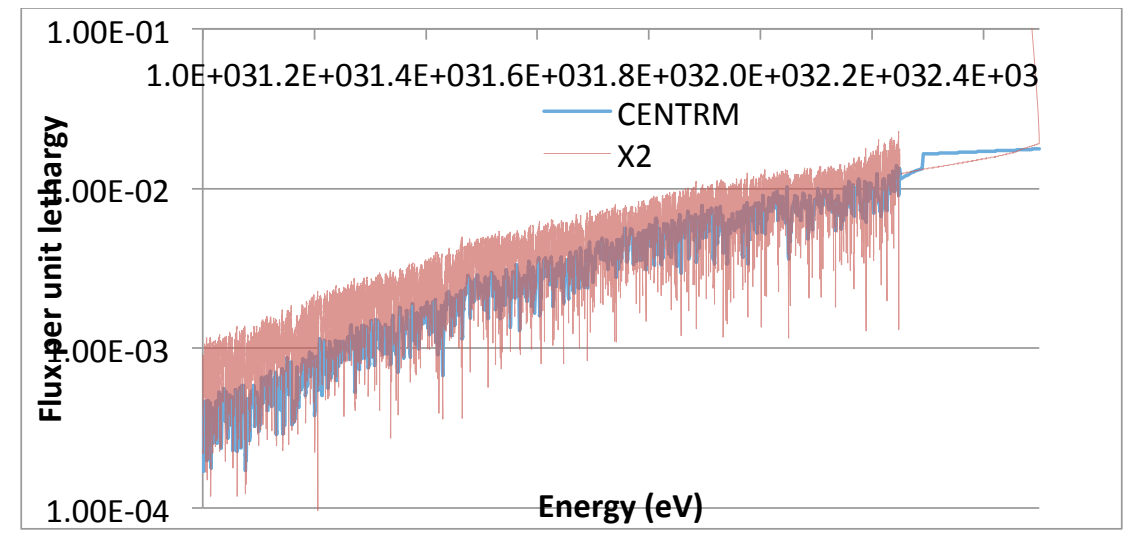

Fig. 6a. Comparison of fluxes in X2 and CENTRM with user-specified source capability $[1000 \mathrm{eV}, 2200 \mathrm{eV}]$.

As can be seen from the figure the flux comparison (normalized differently than in Fig. 5a) has significantly improved with the user-specified source capability in X2. As one would expect, the sources being defined similarly in both problems is important for a true comparison.

As done previously, we re-ran $\mathbf{X} \mathbf{2}$ with the added capability at lower energies. As expected, the results, shown below in Fig. $6 \mathrm{~b}$ are similar to those previously.

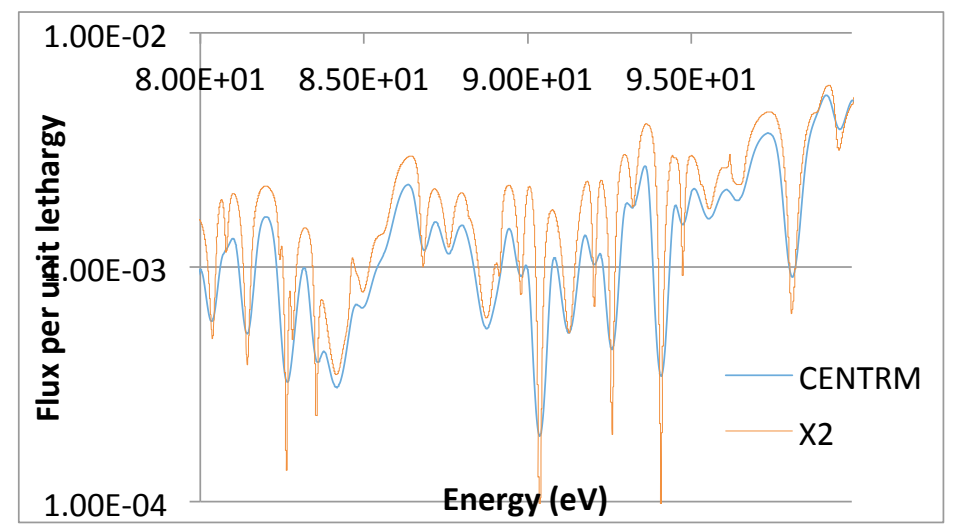

Fig. 6b: Comparison of fluxes in X2 and CENTRM with user-specified source capability $[80 \mathrm{eV}, 100 \mathrm{eV}]$.

While we do not achieve relative errors representative of analytical benchmarks, we do see graphical agreement between the two different slowing down formulations. The flux distributions generally follow the resonance shapes relatively closely. Given data variability and that the algorithms were developed entirely independently, better agreement should not be expected. 


\subsection{X2/MCNP comparison}

For further confirmation, a Monte Carlo calculation was performed with MCNP5 to compare to the results of our UfG code. "Energy bins" were placed in MCNP5 to estimate the flux as a function of energy. The results of this comparison are presented in Fig. 7. Again, general agreement is noted.

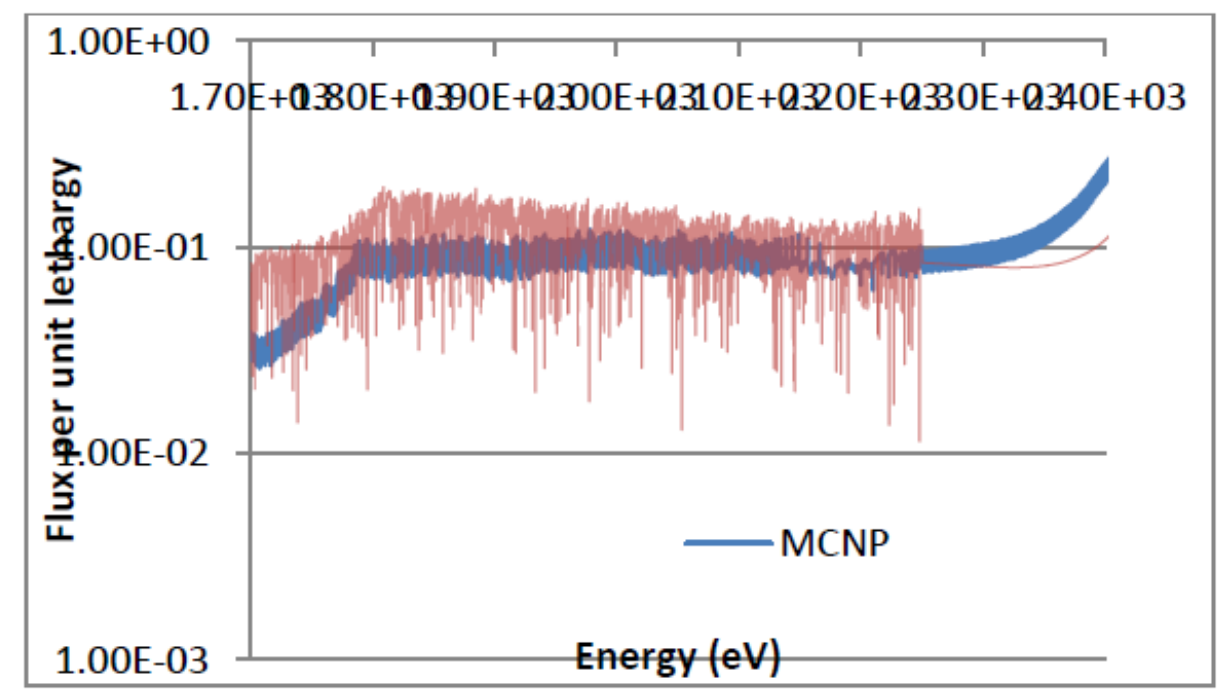

Fig.7. X2/MCNP5 comparison.

\subsection{Summary}

One conclusion from the CENTRM verification study is that no better than a graphical comparison is possible. Benchmarking to more than two digits will not be possible because of several reasons. Most prominent among these is that the cross section data is simply too variable. Also, group generation is different between the two calculations with the $\mathbf{X} 2$ generated set most likely more accurate than that of SCALE. 
II.5. TASK 5 Creation and testing of the diffusion multigroup algorithm in spherical and cylindrical geometries.

(A17.Analytic M\&C MG Diffusion Paper, A18.Analytic M\&C MG Pres) 5.1 Analytical fine-group solution in spherical and cylindrical geometries For this task, the analytical diffusion solutions in spherical and cylindrical geometries are derived. These geometries are appropriate for pebble bed fueled and conventional cylindrical fuel rodded reactors. Here, we test only the analytical solution in cylindrical geometry for the multigroup/fine-group approximation. In addition, and more of theoretical interest, the finite difference solution to the multigroup cylindrical diffusion formulation is presented and shown to limit to the analytical solution.

\section{a. Analytical diffusion solutions}

\section{a1. Spherical and cylindrical geometries}

Consider the general diffusion equation in a homogeneous region $j$ of a heterogeneous medium

$$
\left[\boldsymbol{D}_{j} \boldsymbol{\nabla}^{2}-\boldsymbol{\Sigma}_{j}\right] \boldsymbol{\phi}_{j}(x)+\left[\boldsymbol{\chi} \boldsymbol{v} \boldsymbol{\Sigma}_{f j}^{T}+\boldsymbol{\Sigma}_{s j}\right] \boldsymbol{\phi}_{j}(x)=-\boldsymbol{Q}_{j}(x)
$$

with the usual nuclear data in matrix form

$$
\begin{aligned}
& \boldsymbol{D}_{j} \equiv \operatorname{diag}\left\{D_{g j} ; g=1, \ldots, G\right\} \\
& \boldsymbol{\Sigma}_{j} \equiv \operatorname{diag}\left\{\Sigma_{g j} ; g=1, \ldots, G\right\} \\
& \boldsymbol{\Sigma}_{s j} \equiv\left\{\Sigma_{g g^{\prime} j} ; g, g^{\prime}=1, \ldots, G\right\} \\
& \chi \boldsymbol{v} \Sigma_{f j}^{T} \equiv\left\{\chi_{g} v \Sigma_{f g^{\prime} j} ; g, g^{\prime}=1, \ldots, G\right\}
\end{aligned}
$$

and the group flux and source vectors

$$
\boldsymbol{\phi}_{j}(x) \equiv\left[\begin{array}{l}
\phi_{1 j}(x) \\
\phi_{2 j}(x) \\
\phi_{3 j}(x) \\
\cdots \\
\phi_{G j}(x)
\end{array}\right] \quad \boldsymbol{Q}_{j}(x) \equiv\left[\begin{array}{l}
Q_{1 j}(x) \\
Q_{2 j}(x) \\
Q_{3 j}(x) \\
\cdots \\
Q_{G j}(x)
\end{array}\right]
$$


The analytical solution obtained by diagonalization of the interaction matrix (A19.Anal.MG) is

$$
\begin{aligned}
\boldsymbol{\phi}_{j}(x) & =\boldsymbol{\alpha}_{j} \boldsymbol{h}_{j}^{+}(x) \boldsymbol{\alpha}_{j}^{-1}\left[\boldsymbol{\phi}_{j}-\boldsymbol{\phi}_{P j}\left(x_{j}\right)\right]+ \\
& +\boldsymbol{\alpha}_{j} \boldsymbol{h}_{j}^{-}(x) \boldsymbol{\alpha}_{j}^{-1}\left[\boldsymbol{\phi}_{j-1}-\boldsymbol{\phi}_{P j}\left(x_{j-1}\right)\right]+\boldsymbol{\phi}_{P j}(x)
\end{aligned}
$$

For down scatter and no fission neutrons appearing in the energy region of interest, the eigenvector matrix takes the following form:

$$
\alpha_{j} \equiv\left[\begin{array}{ccccc}
1 & 0 & \ldots & \ldots & 0 \\
\alpha_{21}^{j} & 1 & 0 & \ldots & \ldots \\
\ldots & \ldots & \ldots & \ldots & \ldots \\
\ldots & \ldots & \ldots & 1 & 0 \\
\alpha_{G 1}^{j} & \ldots & \ldots & \ldots & 1
\end{array}\right]
$$

With $\boldsymbol{B}_{j}^{2}$ as the eigenvalue matrix of the interaction matrix diagonalization

$$
\boldsymbol{B}_{j}^{2} \equiv \operatorname{diag}\left\{B_{j k}^{2} ; k=1, \ldots, G\right\}
$$

the independent solutions to the diffusion equation are

$$
\boldsymbol{h}_{j}^{ \pm}(x) \equiv \operatorname{diag}\left\{h_{j k}^{ \pm}(x) ; k=1, \ldots G\right\}
$$

or rearranged in terms of primitive solutions

$$
\begin{aligned}
& h_{j k}^{+}(x) \equiv\left[\frac{\Phi_{h, j}^{-}\left(B_{j k} x_{j-1}\right) \Phi_{h, j}^{+}\left(B_{j k} x\right)-\Phi_{h, j}^{+}\left(B_{j k} x_{j-1}\right) \Phi_{h, j}^{-}\left(B_{j k} x\right)}{\Phi_{h, j}^{+}\left(B_{j k} x_{j}\right) \Phi_{h, j}^{-}\left(B_{j k} x_{j-1}\right)-\Phi_{h, j}^{+}\left(B_{j k} x_{j-1}\right) \Phi_{h, j}^{-}\left(B_{j k} x_{j}\right)}\right] \\
& h_{j k}^{-}(x) \equiv\left[\frac{\Phi_{h, j}^{+}\left(B_{j k} x_{j}\right) \Phi_{h, j}^{-}\left(B_{j k} x\right)-\Phi_{h, j}^{-}\left(B_{j k} x_{j}\right) \Phi_{h, j}^{+}\left(B_{j k} x\right)}{\Phi_{h, j}^{+}\left(B_{j k} x_{j}\right) \Phi_{h, j}^{-}\left(B_{j k} x_{j-1}\right)-\Phi_{h, j}^{+}\left(B_{j k} x_{j-1}\right) \Phi_{h, j}^{-}\left(B_{j k} x_{j}\right)}\right] .
\end{aligned}
$$


For 1D plane, spherical and cylindrical geometries $\Phi_{h, j}^{ \pm}$are the independent solutions to Helmholtz equation appropriate to each geometry. Specifically, these are for

a. Spherical geometry

$$
\Phi_{h, j}^{ \pm}(x)=\frac{B_{j k}}{x}\left\{\begin{array}{l}
\sin (x) \\
\cos (x)
\end{array}\right.
$$

b. Cylindrical geometry in the central region,

$$
\Phi_{h, j}^{ \pm}(x)=\left\{\begin{array}{l}
J_{0}(x) \\
Y_{0}(x),
\end{array}\right.
$$

or for the outer regions

$$
\Phi_{h, j}^{ \pm}(x)=\left\{\begin{array}{l}
H_{0}^{(1)}(x) \\
H_{0}^{(2)}(x),
\end{array}\right.
$$

where $J, Y, H$ are Bessel functions of the first, second and third kinds. Note that $\boldsymbol{h}_{j}^{ \pm}(x)$ has been constructed to be

$$
\begin{aligned}
\boldsymbol{h}_{j}^{+}\left(x_{j}\right) & =1, \boldsymbol{h}_{j}^{+}\left(x_{j-1}\right)=0 \\
\boldsymbol{h}_{j}^{-}\left(x_{j}\right) & =0, \boldsymbol{h}_{j}^{-}\left(x_{j-1}\right)=1 .
\end{aligned}
$$

The particular solution $\boldsymbol{\phi}_{P j}(x)$ is constant for an assumed constant source over region $j$. The conditions at material boundaries determine the interfacial fluxes $\boldsymbol{\phi}_{j-1}$ and $\boldsymbol{\phi}_{j}$.

\section{a2. Numerical demonstration in cylindrical geometry}

Now for a demonstration of the evaluation of the analytical solution in cylindrical geometry. All the input detail is presented in order to serve as a reference for the deliverable and future developments. 
Essentially, the same evaluation procedure is used as for plane geometry with the program flow chart reproduced in Fig. 1 for convenience. A 6-region cylindrical

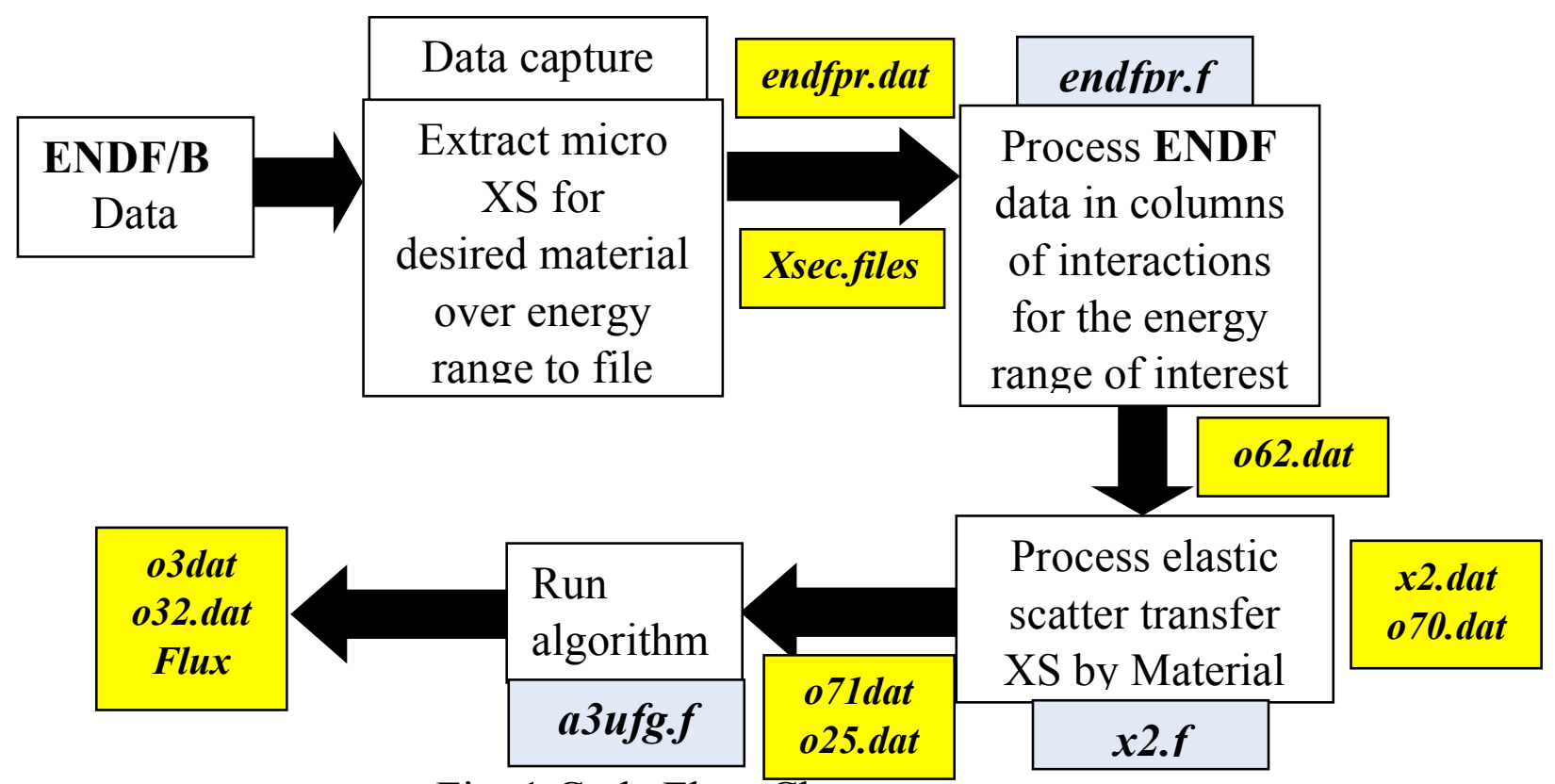

Fig. 1 Code Flow Chart

core composed of alternating fuel and water will be simulated as shown in Fig. 2.

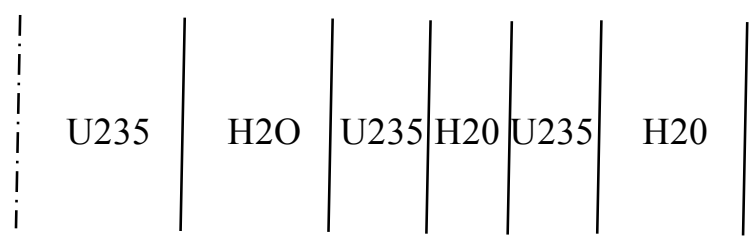

Fig. 2. Simulated cylindrical core.

The following is the detailed input to the modified $\boldsymbol{a} \mathbf{u} \boldsymbol{u f g} . \boldsymbol{f}$ :

1. Make Xsec.files for energy range of interest from ENDF/B

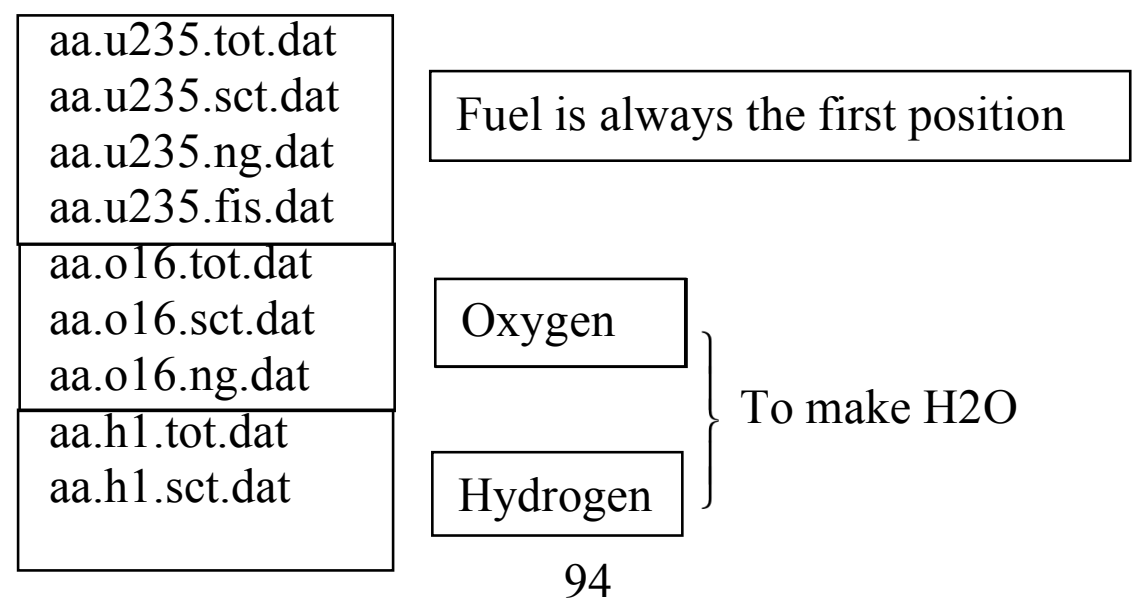


aa.h1.ng.dat

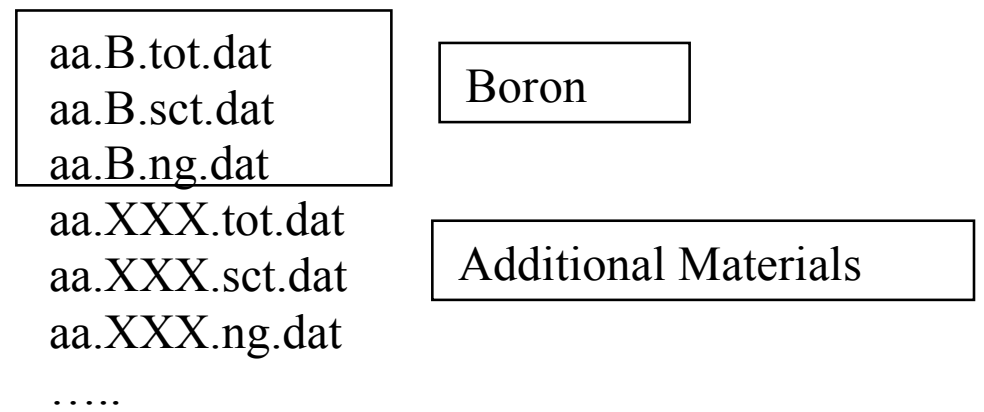

Note that Boron has been include to be able to add sufficient absorption if the matrix diagonalization becomes unstable.

2. endfpr.f: Xsec processing endfpr.dat

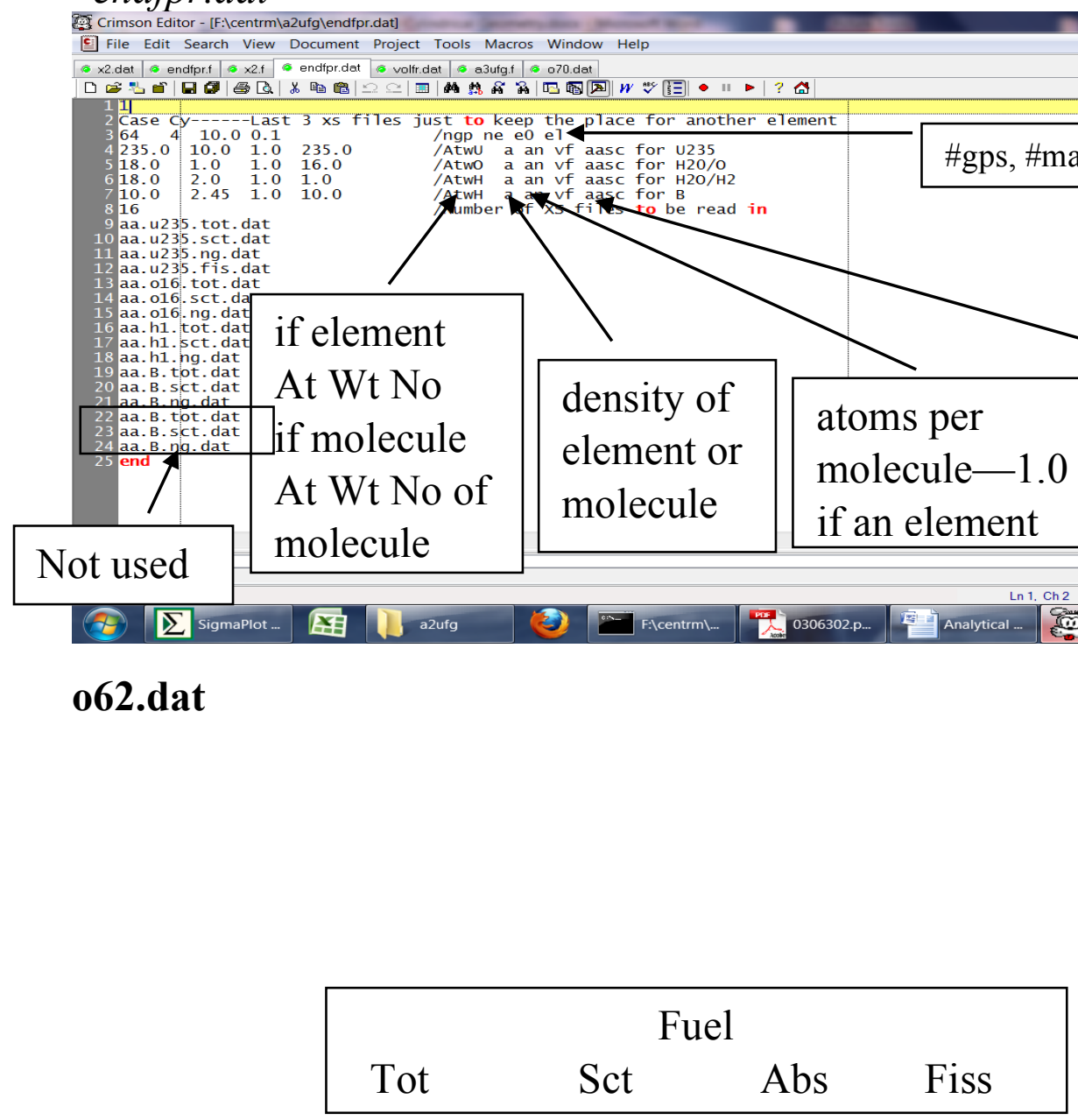




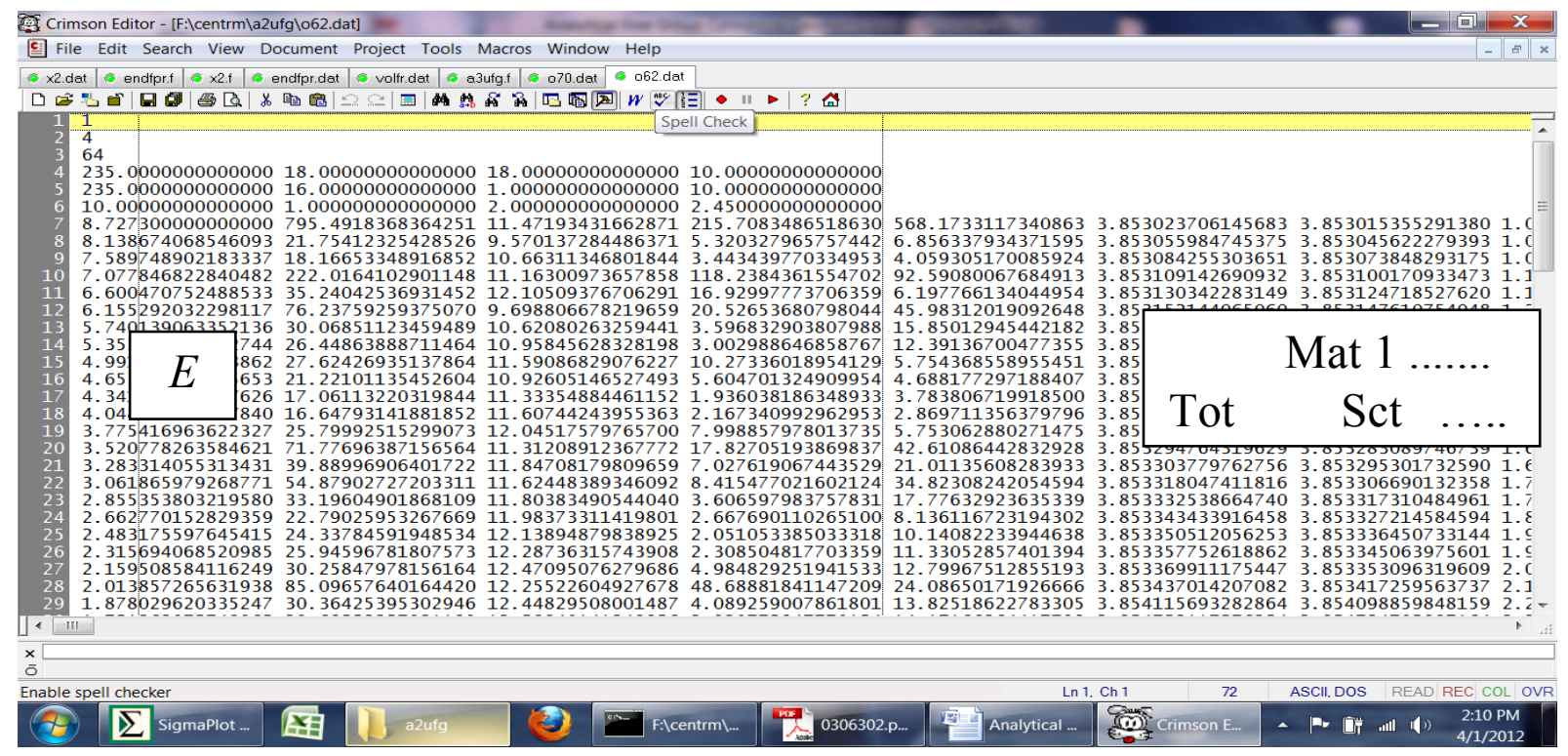

\section{3. x2.f: UfG Processor}

\section{x2.dat}

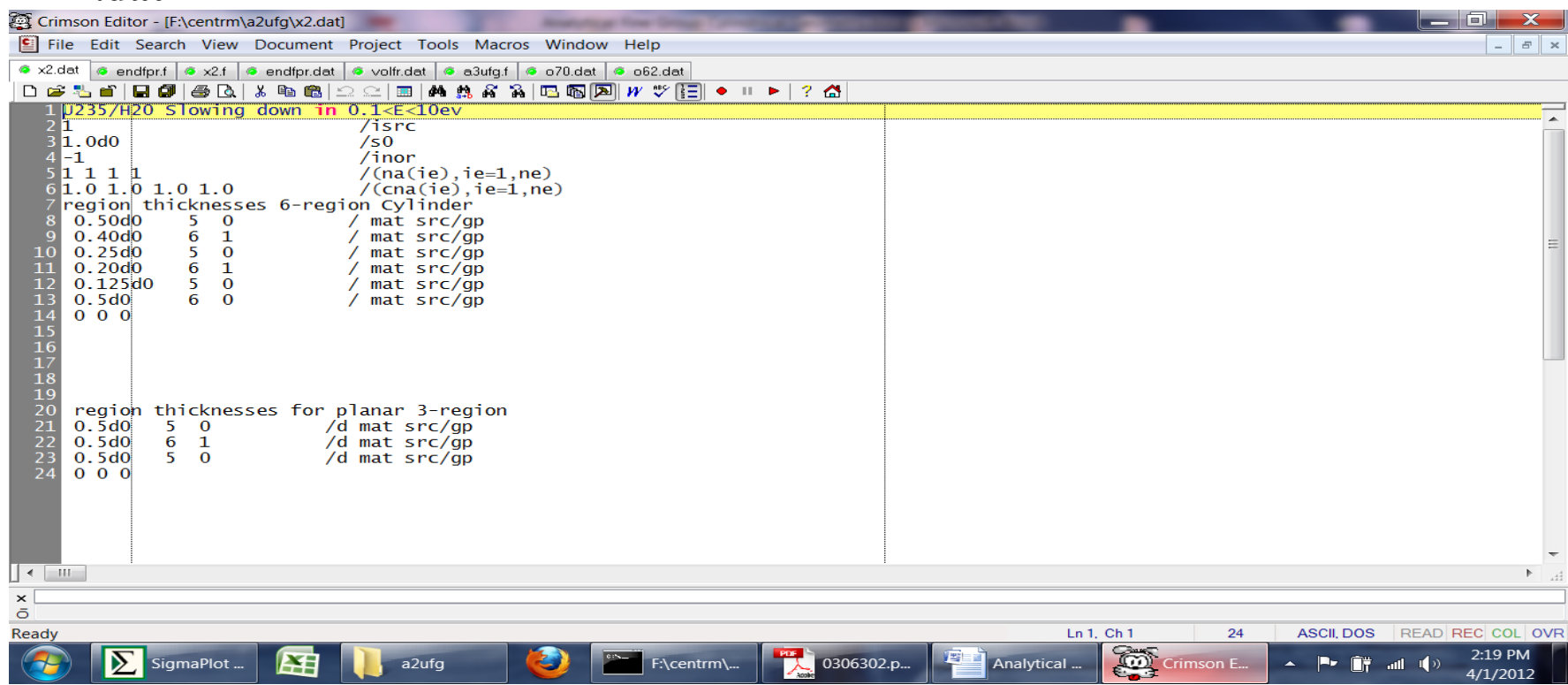

\section{o70.dat (Input in $\boldsymbol{a} 3 \boldsymbol{u} \boldsymbol{f g}$.f)}

This file becomes the header to o71.dat

2

$-1$
$/ 1 \mathrm{pl} / 2 \mathrm{cy} / 3$

/lapack

$\begin{array}{llllll}-1 & -1 & -1 & -1 & 3 & / \text { ng itml itmr nor ick isp(3) }\end{array}$

1.0d-10 $25 \quad 1 \quad$ /err,ns,litr
1.00 .0
/ak, ra (Not used)

$\mathrm{xx}$ gp test case for diagonalization

transfer cross sections

o71.dat: Note a 4-gp example—specifies all Xsec and region bdrys to solver 


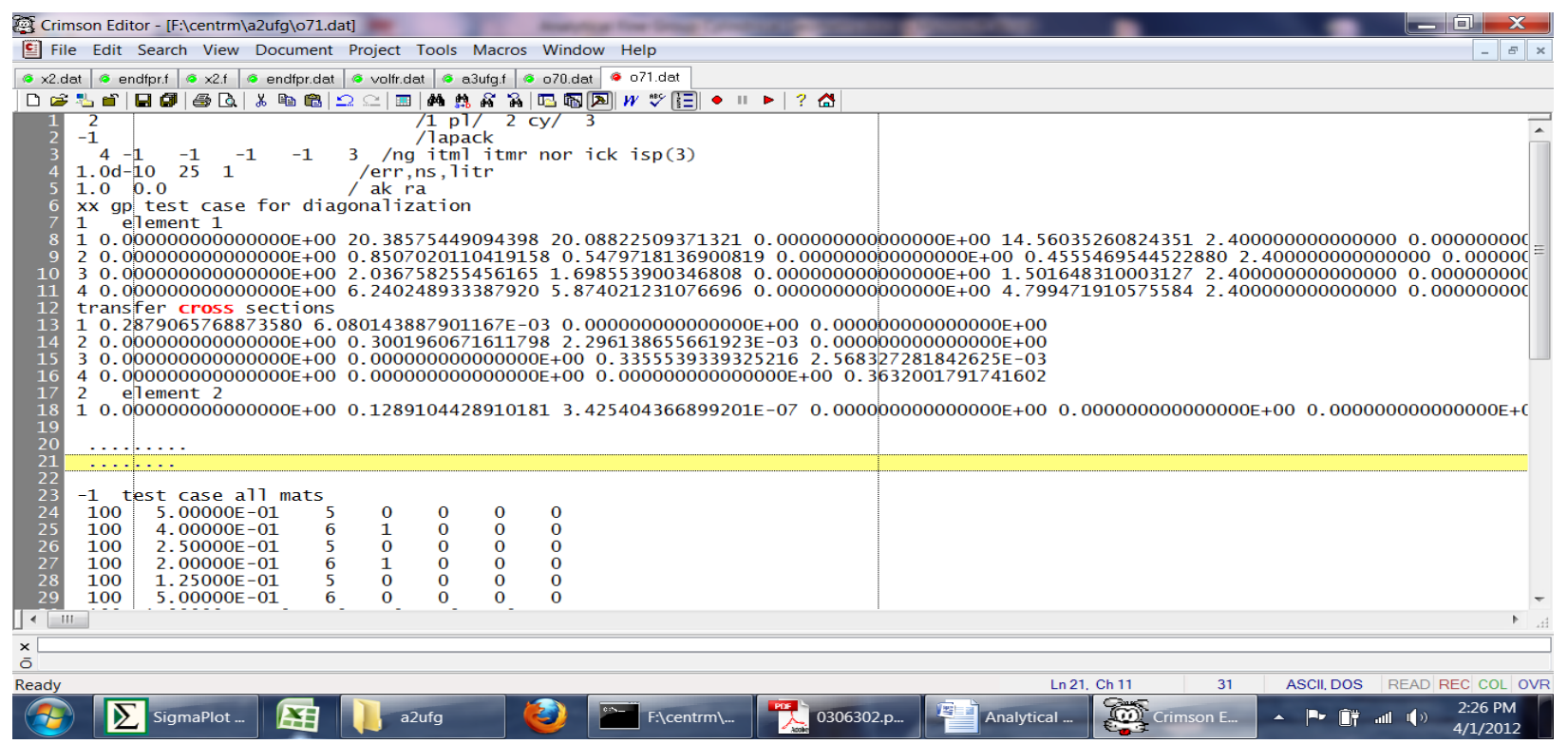

volfr.dat: Specifies volume fractions for including Boron

$\begin{array}{lllll}1.0 & 0.0 & 0.0 & 0.00 & \text { af/Mat5: fuel/O:H/B }\end{array}$

0.00 .990 .990 .01 af/Mat6: fuel/O:H/B

The four entries indicate the composition of materials 5 and 6 with B. The entries are the volume fractions for fuel, $\mathrm{O}, \mathrm{H}$ and $\mathrm{B}$. For this case, the densities of $\mathrm{O}$ and $\mathrm{H}$ have been prepared to give consistent atom densities for water on file endfpr.dat.
4. o3.dat: Output
$\mathrm{x} / \mathrm{Gp} \quad 1$
2
3
4
5
$0.000000 \quad 52.42600 \mathrm{e}-9 \quad 0.012098 \quad 0.011721 \quad 359.5900 \mathrm{e}-6 \quad 7.300400 \mathrm{e}-3 \ldots$
$\begin{array}{lllllll}0.020000 & 59.09300 \mathrm{e}-9 & 0.012099 & 0.011721 & 362.2700 \mathrm{e}-6 & 7.301600 \mathrm{e}-3 \ldots\end{array}$
$\begin{array}{llllll}0.040000 & 81.65400 \mathrm{e}-9 & 0.012101 & 0.011722 & 370.3800 \mathrm{e}-6 & 7.305300 \mathrm{e}-3 \ldots\end{array}$
$0.060000 \quad 128.8900 \mathrm{e}-9 \quad 0.012104 \quad 0.011724 \quad 384.1600 \mathrm{e}-6 \quad 7.311500 \mathrm{e}-3 \ldots$
$\begin{array}{llllll}0.080000 & 219.5600 \mathrm{e}-9 & 0.012109 & 0.011726 & 403.9800 \mathrm{e}-6 & 7.320300 \mathrm{e}-3 \ldots\end{array}$
$\begin{array}{llllll}0.100000 & 390.6100 \mathrm{e}-9 & 0.012115 & 0.011729 & 430.4000 \mathrm{e}-6 & 7.331500 \mathrm{e}-3 \ldots\end{array}$
$\begin{array}{llllll}0.120000 & 713.3400 \mathrm{e}-9 & 0.012123 & 0.011732 & 464.1700 \mathrm{e}-6 & 7.345200 \mathrm{e}-3 \ldots\end{array}$
$\begin{array}{llllll}0.140000 & 1.325200 \mathrm{e}-6 & 0.012132 & 0.011736 & 506.2500 \mathrm{e}-6 & 7.361400 \mathrm{e}-3 \ldots\end{array}$

o3.dat: SigmaPlot ${ }^{T M}$ file--giving 


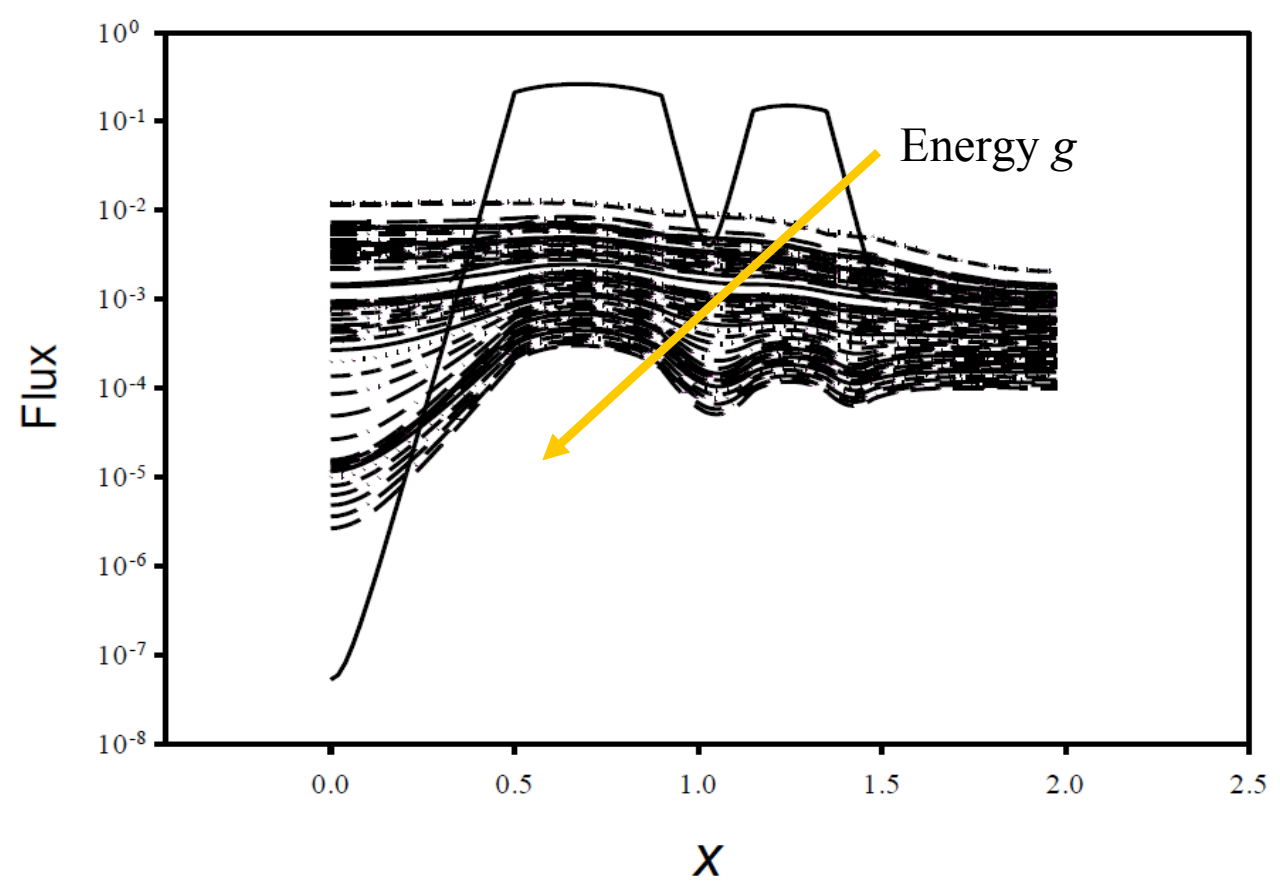

for all 64 groups with uniform sources in the water channels.

\section{b. Analytical FD approximation in cylindrical geometry (A20.AFD Approximation)}

The numerical workhorse for computing the flux from diffusion theory is the FD approximation as demonstrated in §II.3.1. Usually, once one establishes the approximation, little thought is given to its solution other than its numerical implementation. Because of the lack of attention to the analytical finite difference solution, valuable numerical information is lost. Here, our intent is to outline a FD algorithm first and then seek its analytical solution through a limit afterwards.

\section{b1. The FD algorithm}

In fundamental form, the multigroup equation in cylindrical geometry is

$$
\left[I \frac{1}{r} \frac{d}{d r} r \frac{d}{d r}+B_{j}^{2}\right] \boldsymbol{\phi}_{j}(r)=-\boldsymbol{D}_{j}^{-1} \boldsymbol{Q}_{j}(r) .
$$

On diagonalization of the $\boldsymbol{B}_{j}^{2}$ matrix, we arrive at the diagonalized one-group equations for a homogeneous 1D cylindrical medium (subscript $j$ is dropped) 


$$
\frac{1}{r} \frac{d}{d r}\left(r \frac{d}{d r} \phi(r)\right)+B^{2} \phi(r)=-q(r)
$$

for a general source and where $B^{2}$ are eigenvalues of the interaction matrix of region $j$. The source will not concern us at this point because we are interested only in the solutions to the homogeneous equation since the particular solution comes directly from these.

Forming a central difference approximation gives

$$
(2 j+1) \phi_{j+1}-2 j \alpha \phi_{j}+(2 j-1) \phi_{j-1}=-2 j h q_{j},
$$

where

$$
\alpha \equiv 2-h^{2} B^{2}
$$

Note that we have evaluated the FD approximation at the knots $r=j h$.

Now consider the homogeneous equation associated with $\operatorname{Eq}(2 \mathrm{a})$

$$
(2 j+1) y_{j+1}-2 j \alpha y_{j}+(2 j-1) y_{j-1}=0 \text {. }
$$

Through a series of substitutions and manipulation, one finds two complimentary solutions of Eq(3) [1] (after some algebra)

$$
\begin{aligned}
& y_{j}^{(1)}=\gamma^{-j} \sum_{k=0}^{\infty} \frac{(-1)^{k} \lambda(\alpha)^{k}}{2^{2 k}(k !)^{2}}\left[(j-1 / 2)^{(2 k)}\right] . \\
& y_{j}^{(2)}=\gamma^{-j} \sum_{k=0}^{[(j+1) / 2]} \frac{(-1)^{k} \lambda(\alpha)^{k}}{2^{2 k}(k !)^{2}}(j-1 / 2)^{(2 k)} \psi(j-2 k)+ \\
&+\sum_{k=1}^{\infty} \frac{(-1)^{k-1} \lambda(\alpha)^{k}}{2^{2 k}(k !)^{2}}(j-1 / 2)^{(2 k)} H_{k},
\end{aligned}
$$

where 


$$
\begin{aligned}
& \lambda(\alpha)=\frac{(2-\alpha)(2+\alpha)}{\alpha^{2}} \\
& \gamma=\frac{2}{\alpha} \\
& \psi(j-2 k)=\sum_{j=a}^{j-2 k-1} \frac{1}{j^{\prime}+1 / 2} \\
& H_{k} \equiv \sum_{k=1}^{k} \frac{1}{k^{\prime}} .
\end{aligned}
$$

Hence, Eqs(4) represent two independent solutions of the homogeneous FD equation $[\mathrm{Eq}(2 \mathrm{a})]$ with the general complementary solution

$$
y_{j}=A y_{j}^{(1)}+C y_{j}^{(2)}
$$

to which one adds the particular solution. We now seek the two complementary solutions and their limit to zero discretization.

b2. The limit as $h \rightarrow 0$ and $j \rightarrow \infty$

In both independent solutions, $\lambda$ to order $h^{2}$ is

$$
\lambda(\alpha)=B^{2} h^{2}\left[1+O\left(h^{2}\right)\right]
$$

and

$$
\gamma^{-1}=1-\frac{B^{2} h^{2}}{2}
$$

which, when introduced into $y_{j}^{(1)}$ gives

$$
y_{j}^{(1)}=\sum_{k=0}^{\infty} \frac{(-1)^{k} B^{2 k}}{2^{2 k}(k !)^{2}}\left[1+O\left(h^{2}\right)\right]^{k+j}\left[(h j-h / 2)^{(2 k)}\right] .
$$

In the limit to the true solution with $r \equiv r_{j}=h j$ kept fixed as $h$ and $j$ approach zero and infinity respectively, we find 


$$
\lim _{\substack{h \rightarrow 0 \\ j \rightarrow \infty}} y_{j}^{(1)}=y^{(1)}\left(r_{j}\right)=\sum_{k=0}^{\infty} \frac{(-1)^{k}(B r)^{2 k}}{2^{2 k}(k !)^{2}}=J_{0}(B r) .
$$

Similarly, for the second solution

$$
\begin{aligned}
y_{j}^{(2)}=\sum_{k=0}^{[(j+1) / 2]} & \frac{(-1)^{k} B^{2 k}}{2^{2 k}(k !)^{2}}\left[1+O\left(h^{2}\right)\right]^{k+j}\left(r_{j}-h / 2\right)^{(2 k)} \psi(j-2 k)+ \\
& +\sum_{k=1}^{\infty} \frac{(-1)^{k-1} B^{2 k}}{2^{2 k}(k !)^{2}}\left[1+O\left(h^{2}\right)\right]^{k+j}\left(r_{j}-h / 2\right)^{(2 k)} H_{k},
\end{aligned}
$$

with

$$
\psi\left(r_{j} h-2 k h\right)=\sum_{j^{\prime}=a}^{j-2 k-1} \frac{h}{h j^{\prime}+h / 2}=\sum_{r_{j^{\prime}}=r_{a}}^{r_{j}-2 h k-h} \frac{h}{r_{j^{\prime}}+h / 2} .
$$

The limit of this expression for fixed $r_{j}=r$ is therefore

$$
\lim _{\substack{h \rightarrow 0 \\ j \rightarrow \infty}}[\psi(r h-2 k h)]=\lim _{\substack{h \rightarrow 0 \\ j \rightarrow \infty}} \sum_{r_{j^{\prime}}=r_{a}}^{r-2 h k-h} \frac{h}{r_{j^{\prime}}+h / 2}=\ln \left[\frac{B r}{B r_{a}}\right]
$$

by noting that the sum is a Riemann sum representation of an integral and introducing a multiplicative factor $B$. Note that the lower limit is arbitrary.

Finally, on substitution into the second solution

$$
y^{(2)}(r)=\ln \left[\frac{r}{r_{a}}\right] \sum_{k=0}^{\infty} \frac{(-1)^{k}(B r)^{2 k}}{2^{2 k}(k !)^{2}}+\sum_{k=1}^{\infty} \frac{(-1)^{k-1}(B r)^{2 k}}{2^{2 k}(k !)^{2}} H_{k}
$$

and identifying the first sum as the first kind Bessel function gives

$$
y^{(2)}(r)=\left[\ln (B r)-\ln \left(B r_{a}\right)\right] J_{0}(B r)+\sum_{k=1}^{\infty} \frac{(-1)^{k-1}(B r)^{2 k}}{2^{2 k}(k !)^{2}} H_{k} \text {. }
$$


To conform to the Weber form of the Bessel function of the second kind, we let

$$
\ln \left(B r_{a}\right) \equiv-\gamma+\ln (2)
$$

and set the constant $C_{1}$ to $2 / \pi$ to give

$$
y^{(2)}(r)=\frac{2}{\pi}\left\{\begin{array}{l}
{\left[\ln \left(\frac{B r}{2}\right)+\gamma\right] J_{0}(B r)+} \\
+\sum_{k=1}^{\infty} \frac{(-1)^{k-1}(B r)^{2 k}}{2^{2 k}(k !)^{2}} H_{k}
\end{array}\right\}=Y_{0}(B r)
$$

where $\gamma$ is the Euler-Mascheroni constant.

While the analytical solutions just found can be directly obtained simply by inspection of the diffusion equation in cylindrical geometry, the value of the above analysis is the challenge of developing a complete solution from discretization alone. That is, we start from a basic FD approximation and arrive at the analytical solution in the limit. In this way, one develops numerical approximations based on several levels of the FD form. The levels are the numerical evaluations of the FD approximation of $\operatorname{Eq}(2 a)$ itself, Eqs(4) or the analytical solution of $\operatorname{Eqs}(6)$. This is particularly important in heterogeneous media in the multigroup approximation. Since the findings of our research thus far indicate that for the UfG panel algorithm, the analytical solution is far too costly for practical application, it becomes necessary to design a highly accurate FD algorithm. We now have several options from which to choose in cylindrical geometry, which leads into the final task.

\section{II.6. TASK 6 Burnup mapping with analytical diffusion theory}

\subsection{Burnup application}

We introduce the final task to be a representative application of the basic research performed thus far. Hence, a Ph.D. dissertation was proposed for R. Joseph pertaining to the development of a multigroup burnup benchmark. Burnup in conjunction with the analytical diffusion solution and the isotopics code ORIGIN [1] in cylindrical geometry was thought to be a new and therefore, an appropriate topic to showcase the results or our NEUP grant. 
With the completion of the analytical solution in heterogeneous cylindrical geometry, it becomes possible to consider a relatively realistic application. Thus, in this task we consider burnup in a cylindrical fuel pin. We anticipate the advantage of applying the analytical solution is its ability to treat multigroup diffusion in a highly heterogeneous medium. We envision the average cylindrical fuel rod to be segmented into concentric cylindrical regions, where each region is representative of a burnup level. This would mean that each concentric cylindrical would contain different isotopes, which could easily be handled by the analytical formalism. The analysis would necessarily require time be added in some fashion as well as an isotropic generator like ORNL's ORIGIN. Initially, we will consider a quasi-static approach, with isotopic updating at the end of each time step. One could also envision a full time dependent calculation in the future however.

Initial progress has been made in becoming familiar with ORIGIN through study of the manual and performing some tests. One of the issues that had to be resolved is how to specify an input flux in ORIGEN instead of a burnup. A second difficulty encountered was how to handle the changed flux spectrum after each run of the analytical solution, now called the Analytical Diffusion Code (ADC). These issues have now been resolved. We can transfer the final flux energy map after running the ADC as the initiating source to the next time step using ADC to advance the burnup cycle. In addition, one of the main tasks was to modify ADC to input cross sections for more than six materials. The code modifications include allowing input of volume fractions for more than 4 materials, modifying the input file to input how many base materials and mixed materials are in the problem, and other general modifications of calculations needed because of the unknown number of materials coming from each burn cycle. The code has been partially tested for computational consistency but unfortunately, further testing will be delayed indefinitely because of the termination of the $\mathbf{U f G}$ project.

\section{REFERENCES}

\section{PROJECT BACKGROUND/SUMMARY/HISTORY}

1. M.L. Williams, M. Asgari, and D.F. Hollenbach, "CENTRM: A OneDimensional Neutron Transport Code for Computing Pointwise Fluxes," Vol. II, Sect. F18, NUREG/CR-0200, Rev. 7 (ORNL/NUREG/CR/CSD-2R7), 3 vols., April 2004. 
2. SCALE: A Modular Code System for Performing Standardized Computer Analysis for Licensing Evaluation, NUREG/CR-0200, Rev. 7 (ORNL/NUREG/CR/CSD-2R7), 3 vols., April 2004.

3. B.D. Ganapol, W. Yoon and D.W. Nigg, Verification of the COMBINE7 Neutron Energy Spectrum Code, INL Progress Report (2007).

4. B.D. Ganapol, Benchmarks in Nuclear Engineering, OECD, 2008.

\section{TASK 1}

1. V. Fairen, et al., "Power Series Approximation to Solutions of Nonlinear Systems of Differential Equations", Am J. Phys., 56, 1 (1988).

2. B.D. Ganapol, Benchmarks in Nuclear Engineering, OECD, 2008.

3. W. Press, et. al., Numerical Recipes, Cambridge Univ. Press, London, UK, (1992).

\section{TASK 2}

1. NJOY: http://t2.lanl.gov/nis/njoy/njoy01.html

\section{TASK 3}

1. X.D. Fu and N.Z. Cho, Jour of Nucl. Sci. and Tech., V39,No.10, 1015-1022 (2002).

2. A. Hébert, Applied Reactor Physics, Presses Internationaes, Polytechnique, 2009.

3. B.D. Ganapol, Benchmarks in Nuclear Engineering, OECD, 2008.

4. W. Press, et. al., Numerical Recipes, Cambridge Univ. Press, London, UK, (1992).

5. LAPACK, http://www.netlib.org/lapack/

6. B.D. Ganapol and D.W. Nigg, Extension of the 1D Analytic Nodal Method to Multigroup INL Progress Report 2, July 2007.

7. A. Sood, A. Forster and K. Parsons, Analytical Benchmarks Test Set for Criticality Code Verification, Prog., in Nucl. Ener., 42, 55(2003).

\section{TASK 4}

1. M.L. Williams, M. Asgari, and D.F. Hollenbach, "CENTRM: A OneDimensional Neutron Transport Code for Computing Pointwise Fluxes," Vol. II, Sect. F18, NUREG/CR-0200, Rev. 7 (ORNL/NUREG/CR/CSD-2R7), 3 vols., April 2004.

2. SCALE: A Modular Code System for Performing Standardized Computer Analysis for Licensing Evaluation, NUREG/CR-0200, Rev. 7 (ORNL/NUREG/CR/CSD-2R7), 3 vols., April 2004. 


\section{TASK 5}

1. H. Levy and F. Lessman, Finite Difference Equations, Dover, 1961.

\section{TASK 6}

1. ORIGIN: http://www.oecd-nea.org/tools/abstract/detail/ccc-0371 


\section{MODIFIED TASK COMPLETION PERCENTAGES}

\section{TASK 1: Status: $100 \%$}

TASK 2: Status: $\mathbf{1 0 0 \%}$

TASK 3: Status: $75 \%$

While the status of Task 3 is not $100 \%$ complete, significant strides have been made. In particular, multigroup/fine-group forms for both analytical and finite difference diffusion theory as well as multigroup/fine-group transport theory have been established. This is a necessary first step in developing the UfG panel formalism. The next step is to enable the panel formulation both theoretically and computationally. This requires analytical forms for the particular solution in the case of diffusion theory. It is also clear that to proceed, HPC will be required. This was the primary factor in purchasing large and small exploratory GPUs, which eventually will be programmed for the panel method.

\section{TASK 4: Status: $100 \%$}

TASK 5: Status: $100 \%$

TASK 6: Status: $10 \%$

This task was begun late in the fourth year. The parameters of the dissertation have been defined; but the task has been postponed indefinitely, since the grant has ended. Robby Joseph has returned full time to his ORNL position. This task may or may not ever be completed. If not, the fault is clearly attributable to the short sightedness of the DoE manager. 


\section{PERSONNEL}

Co-PI (UA):

Dr. C. Chan

Professor

Department of Aerospace and Mechanical Engineering

University of Arizona

Dr. I. Guven

Assistant Professor

Department of Materials Science

University of Arizona

\section{Co-PI (UTK):}

Dr. I. Maldonado

Associate Professor

Department of Nuclear Engineering

University of Tennessee

\section{Graduate Students:}

A. Farber

Graduate Student (hourly)

AME Department

University of Arizona

R. Joseph

Graduate Student

Nuclear Engineering Department

University of Tennessee

\section{Undergraduates (UA):}

Blake Tye (Freshman/Undecided)

Allison Sheesley (Freshman/Undecided)

Michael Futch (Freshman/MSE)

Matthew Avitian (Senior/AME)

Angel Tellez (Senior/AME)

Visiting Scholars (Italy):

Elia Battistini

Department of Energy Systems

University of Bologna

Italy 


\begin{abstract}
N. Terranova
Department of Energy Systems University of Bologna

Italy
\end{abstract}




\section{PUBLICATIONS AND PRESENTATIONS}

\section{Publications}

1. B.D. Ganapol and D.W. Nigg, High Order Finite Difference Approximations to the One-Group Neutron Diffusion Equation in 1D Heterogeneous Media Part I: Theory In Plane Media, PHYSOR2010.

2. B.D. Ganapol and D.W. Nigg, High Order Finite Difference Approximations to the One-Group Neutron Diffusion Equation in 1D Heterogeneous Media Part II: Implementation and Application, PHYSOR2010.

3. E. Battistini, Semi-Analytical Benchmark for Neutron Slowing Down in Multigroup Transport and Diffusion Theory, Thesis, University of Bologna, 2010.

4. E. Battistini, et. al., Paneling Strategy for Ultra-Fine-Group Slowing Down Numerical Benchmarks, ANS Winter 2010 Mtg.

5. E. Battistini, et. al., Convergence Acceleration for Multipanel Fine-group Finite Difference Numerical Benchmarks, ANS Winter 2010 Mtg.

6. B D. Ganapol, et. al., Implementation of an Analytical Multigroup Diffusion Solution in 1D Heterogeneous Media, M\&C Topical Mtg., Brasil, 2011.

7. B.D. Ganapol, et. al., The Solution of the Point Kinetics Equations via Converged Accelerated Taylor Series (CATS), PHYSOR2012, Knoxville TN, April 2012.

8. R.A. Joseph, III, et. al., The Ultra-Fine-Group Panel Method for Neutron Slowing Down, Proceedings of the ICONE20-POWER2012, Anaheim CA, Aug. 2012.

9. N. Terranova and B.D. Ganapol, An Adaptive Natural Multigroup for Neutron Slowing Down, ANS Proceedings Chicago, June 2012.

10. N. Terranova, Numerical Benchmarks for Multigroup Diffusion Theory, MS Thesis, University of Bologna, 2012.

11. B.D. Ganapol, Chandrasekhar Polynomials and the Solution to the Transport Equation in an Infinite Medium, TTSP, submitted.

\section{Presentations}

1. B.D. Ganapol and D.W. Nigg, High Order Finite Difference Approximations to the One-Group Neutron Diffusion Equation in $1 D$ Heterogeneous Media Part I: Theory In Plane Media, PHYSOR2010.

2. B.D. Ganapol and D.W. Nigg, High Order Finite Difference Approximations to the One-Group Neutron Diffusion Equation in 1D Heterogeneous Media Part II: Implementation and Application, PHYSOR2010.

3. B.D. Ganapol, Paneling Strategy for Ultra-Fine-group Slowing Down Numerical Benchmarks, ANS Winter $2010 \mathrm{Mtg}$. 
4. B.D. Ganapol, Convergence Acceleration for Multipanel Fine-group Finite Difference Numerical Benchmarks, ANS Winter 2010 Mtg.

5. B.D. Ganapol, Implementation of an Analytical Multigroup Diffusion Solution in 1D Heterogeneous Media (Brasil M\&C Topical Mtg).

6. Meeting with M. Williams, I. Maldonado, R. Joseph at ORNL, May 2011.

7. At meeting with R. Joseph at UA December 2011, Knoxville TN.

8. At meeting with R. Joseph and I Maldonado, Tucson AZ, January 2012.

9. PHYSOR2012:Workshop on Convergence Acceleration applied to reactor physics.

10. R.A. Joseph, III, et. al., The Ultra-Fine-Group Panel Method for Neutron Slowing Down, Proceedings of the ICONE20-POWER2012, Anaheim CA, July 2012.

11. B.D. Ganapol, An Adaptive Natural Multigroup for Neutron Slowing Down, ANS Proceedings Chicago, June 2012.

12. I. Maldonado and R.A. Joseph, OECD Short Course Paris, 2012.

13. B.D. Ganapol and I Guven, ICTT23, Sept 2013.

14. B.D. Ganapol, INL Short Course, Summer 2013. 
V. DELIVERABLES (Upon Request)

1. UfG/X2 GUI

Automated cross section generation

X2.f/ENDFPR.f

GUI, The Manual

GUI, The Movie

2. 1D/Analytical diffusion solution (Plane, spherical, cylindrical geometries)

a3ufg.folder

MATLAB.folder

3. 1D/FD diffusion solution

Converged accelerated FD solution

4. Converged accelerated Sn solution

MGCSn.folder 